\title{
Nurse-led telemonitoring in asthma : process, outcomes and cost-effectiveness
}

Citation for published version (APA):

Willems, D. C. M. (2008). Nurse-led telemonitoring in asthma : process, outcomes and cost-effectiveness. [Doctoral Thesis, Maastricht University]. Universiteit Maastricht. https://doi.org/10.26481/dis.20080131dw

Document status and date:

Published: 01/01/2008

DOI:

$10.26481 /$ dis.20080131dw

Document Version:

Publisher's PDF, also known as Version of record

\section{Please check the document version of this publication:}

- A submitted manuscript is the version of the article upon submission and before peer-review. There can be important differences between the submitted version and the official published version of record.

People interested in the research are advised to contact the author for the final version of the publication, or visit the DOI to the publisher's website.

- The final author version and the galley proof are versions of the publication after peer review.

- The final published version features the final layout of the paper including the volume, issue and page numbers.

Link to publication

\footnotetext{
General rights rights.

- You may freely distribute the URL identifying the publication in the public portal. please follow below link for the End User Agreement:

www.umlib.nl/taverne-license

Take down policy

If you believe that this document breaches copyright please contact us at:

repository@maastrichtuniversity.nl

providing details and we will investigate your claim.
}

Copyright and moral rights for the publications made accessible in the public portal are retained by the authors and/or other copyright owners and it is a condition of accessing publications that users recognise and abide by the legal requirements associated with these

- Users may download and print one copy of any publication from the public portal for the purpose of private study or research.

- You may not further distribute the material or use it for any profit-making activity or commercial gain

If the publication is distributed under the terms of Article $25 \mathrm{fa}$ of the Dutch Copyright Act, indicated by the "Taverne" license above, 
Nurse-led telemonitoring in asthma

Process, outcomes and cost-effectiveness 
ISBN: 978-90-9022592-0

(C) DCM Willems, Maastricht 2008

Cover design: Roos Huijbrechts

The project presented in this thesis was funded by the Dutch Health Care Insurance Board.

Financial support for printing of this thesis has been provided by

Novartis Pharma B.V., Boehringer Ingelheim, Pfizer B.V.,

The Netherlands Asthma Foundation 


\section{Nurse-led telemonitoring in asthma \\ Process, outcomes and cost-effectiveness}

\section{PROEFSCHRIFT}

ter verkrijging van de graad van doctor aan de Universiteit Maastricht, op gezag van de Rector Magnificus, Prof. mr. GPMF Mols,

volgens het besluit van het College van Decanen, in het openbaar te verdedigen op donderdag 31 januari 2008 om 16.00 uur

door

Daniëlle Catharina Mathias Willems

Geboren op 3 juni 1974 te Herkenbosch-Melick 


\section{Promotores}

Prof. Dr. J.L. Severens

Prof. Dr. E.F.M. Wouters

\section{Co-promotores}

Dr. M.A. Joore

Dr. J.J.E. Hendriks

\section{Beoordelingscommissie}

Prof. dr. L. Zimmermann (voorzitter)

Prof. dr. J.C. de Jongste

Prof. dr. J. Knottnerus

Dr. T.R.J. Schermer

Prof. dr. R. de Wit 
Voor oma 


\section{Contents}

Chapter 1

General introduction and outline of the thesis

9

Chapter 2

The cost-effectiveness of self-management in asthma - a systematic review of peak flow monitoring interventions

\section{Chapter 3}

The process evaluation of a nurse-led telemonitoring programme for asthmatics

Chapter 4

The effectiveness of nurse-led telemonitoring of asthma

\section{Chapter 5}

Using EQ-5D in children with a chronic condition

\section{Chapter 6}

Cost-effectiveness of a nurse-led telemonitoring intervention based on peak expiratory flow measurements in asthmatics: results of a randomised controlled trial

Chapter 7

General discussion

Summary \& Samenvatting

Dankwoord

Curriculum vitae 
Chapter 1

General introduction and outline of the thesis 


\section{Chapter 1}

\section{General introduction}

Asthma

Condition

Asthma is one of the most common chronic diseases. The complete causes of asthma are still unidentified, despite the increase of information about asthma. Heredity does seem to play a role as do allergens and environmental factors. Onset of asthma between the ages of 5-15 years usually indicates asthma with an allergic basis. In the Global Initiative of Asthma (GINA) guidelines of 2006, the operational description of asthma is defined as:

"A chronic inflammatory disorder of the airways in which many cells and cellular elements play a role. The chronic inflammation is associated with airway hyperresponsiveness that leads to recurrent episodes of wheezing, breathlessness, chest tightness, and coughing, particularly at night or in the early morning. These episodes are usually associated with widespread, but variable, airflow obstruction within the lungs that is often reversible either spontaneously or with treatment (1)".

The airways narrow during an asthma attack. The muscles around the airways tighten and mucus clogs the tiny airways in the lungs, making breathing difficult (2). The lining of the bronchial tubes swells, causing the airways to narrow and reducing the flow of air into and out of the lungs (3). Asthma symptoms are often triggered by allergens or airway infections and exercise-induced symptoms are most common in children.

\section{Epidemiology}

An estimated 300 million individuals are affected by asthma worldwide. Its prevalence is increasing in the Western community (4). The prevalence and incidence is the highest among children, where it is the most common chronic disease. With regard to children asthma is more common among male children than female children and with regard to adults asthma is more common among adult women than adult men. In urban areas asthma prevalence is more common compared to rural areas. The ethnic differences in asthma prevalence, morbidity, and mortality are highly correlated with poverty, urban air quality, indoor allergens, and lack of patient education and inadequate medical care. It is estimated that asthma accounts for about one in every 250 deaths worldwide. Many of the deaths are preventable, being due to suboptimal long-term medical care and delay in obtaining help during the final attack (4). The GINA guidelines define two separate age 
categories in the management of asthma, including children of 5 years and younger, and children older than 5 years, adolescents, adults, and elderly (1). This distinction is mostly related to natural history and comorbidities, but also to differences in the diagnostic approach and to the measures for asthma severity and monitoring control.

\section{Burden of disease}

Asthma is under-diagnosed and under-treated, creating a substantial burden to individuals and families and possibly restricting individuals' activities for a lifetime (4). Asthma symptoms, such as breathlessness and wheezing, may cause sleeplessness, daytime fatigue, reduced activity levels and absence from school and work. As a result, the economic burden associated with asthma is considerable (4-6). Although the costs to control asthma from the perspective of both the patient and the society are high, the costs of not treating asthma correctly are even higher (3). Appropriate management of asthma can enable asthma patients to live a normal physically active life with no more medication than actually needed. The costs of asthma depend on the patient's individual level of control and extend to which asthma attacks are avoided. In the year 2000 the societal costs for an asthma patient were on average $€ 313$ in the Netherlands (7). Medication costs were the largest cost component in the health care costs and these costs are expected to increase even further in the future. Other extensive societal costs in asthma care are for instance admission costs and productivity costs. Due to asthma symptoms some asthma patients are not able to work which consequently leads to productivity losses. Absence from work due to self perceived asthma symptoms or due to taking care for an asthmatic child can have enormous financial consequences for both the families and the society. Furthermore, appropriate asthma management will enhance the patient's quality of life (3). The present economic and social burden of asthma underline the relevance of economic evaluations to inform health care decision-makers about asthma management of adults and children.

\section{Asthma management}

\section{Guidelines}

In 1993, the Global Initiative of Asthma was formed (1). The evidence-based GINA guidelines are known worldwide and were shaped by committees made up of leading asthma experts. The goal of GINA is to reduce the asthma prevalence, morbidity, and mortality around the world. At the time of the start of the study underlying this thesis (in the year 2002) the severity of asthma was determined by the combined assessment of symptoms and lung function. It was common practice to take stepwise therapy decisions, 


\section{Chapter 1}

based on the severity of asthma. The asthma severity was classified into Intermittent, Mild Persistent, Moderate Persistent, and Severe Persistent. The presence of one of the features described in table 1 was sufficient to place a patient in that category.

Table 1. Asthma severity classification (GINA 2002)

\begin{tabular}{|c|c|c|c|}
\hline & & & PEF or FEV1 \\
\hline & Symptoms / day & Symptoms / night & PEF variability \\
\hline Step 1 & $<1$ time a week & & \\
\hline \multirow[t]{2}{*}{ Intermittent } & Asymptomatic and normal & $\leq 2$ times a month & $\geq 80 \%$ \\
\hline & PEF between attacks & & $<20 \%$ \\
\hline Step 2 & $>1$ time a week but $>1$ & & \\
\hline \multirow[t]{2}{*}{ Mild persistent } & time a day & $>2$ times a month & $\geq 80 \%$ \\
\hline & Attacks may affect activity & & $20-30 \%$ \\
\hline Step 3 & Daily & & \\
\hline \multirow[t]{2}{*}{ Moderate persistent } & Attacks affect activity & $>1$ time a week & $60-80 \%$ \\
\hline & & & $>30 \%$ \\
\hline Step 4 & Continuous & & \\
\hline \multirow[t]{2}{*}{ Severe persistent } & Limited physical activity & Frequent & $\leq 60 \%$ \\
\hline & & & $>30 \%$ \\
\hline
\end{tabular}

According to the national Guidelines for the Diagnosis and Management of Asthma (7), asthma treatment has four main components:

- The use of an objective measure of lung function (such as peak flow meters and spirometers) to assess the severity of asthma and to monitor the course of treatment

- Environmental control measures to avoid or eliminate factors that trigger asthma symptoms or exacerbations

- Medication therapy for long-term management to reverse and prevent airway inflammation as well as therapy to manage asthma exacerbations

- Patient education to foster a partnership between the patients, his or her family, the physician, and other health care providers

In the GINA Report (1) the goals for successful management of asthma are:

- $\quad$ Minimal or no symptoms, including nighttime symptoms

- $\quad$ Minimal asthma episodes or attacks

- No emergency visits to physicians or hospitals

- Minimal need for reliever medications

- $\quad$ No limitations on physical activities and exercise

- Nearly normal lung function 
- Minimal or no side effects from medication

Asthma management programmes often contain these elements, but as the importance of patient empowerment is increasing recent guidelines recommend self-management for the treatment of asthma (3;8-10). In this thesis self-management is regarded as: the delegation of the surveillance of bodily functions to the patient. Where self-management interventions are techniques for continuous adaptation of treatment, monitoring can play an important role in the way that it constantly diagnoses the severity of asthma (11).

\section{Monitoring}

In self-management programmes home monitoring is essential. The patients perform spirometry tests at home and the data are stored into the monitor. Accordingly, an objective measure of the airway obstruction as an indication of asthma severity takes place. Due to the simplicity of the technique and the potential for unsupervised and repeated measures the peak expiratory flow $(\mathrm{PEF})$ is the most common used spirometry test in home monitoring (12). The PEF rate shows the maximum rate of airflow attained during forced vital capacity determination. Self-management programmes including peak flow monitoring seem to increase patient awareness of their disease status and control (13). Programmes based on PEF can be developed from percent predicted, percent personal best, morning PEF, or average values over time. In many studies home peak flow monitoring in asthma management was found to be the dominant intervention, when compared to an alternative, such as regular care (14-24).

With the use of an electronic monitor in combination with telecommunication techniques care provider are even able to judge the monitor data in only a few seconds in their own work environment. This form of home monitoring is a part of telemedicine, named telemonitoring. These terms will be explained in the next paragraph.

\section{Telemedicine and telemonitoring}

The development of information and communication technologies (ICT) and modern telecommunications provide new opportunities in health care. Consequently, telemedicine is becoming a common concept in health care nowadays. It is defined as the use of electronic ICT to provide and support health care, when distance separates the participants (25). At the moment the term telemedicine is often overtaken by more fashionable terms as online health or e-health (26). In asthma, telemedicine had a great impact, as it changed the way care can be delivered. By telemonitoring care providers are able to monitor the patients from a distance. Lung function can be objectively 


\section{Chapter 1}

assessed outside the office or hospital and can be immediately reviewed. With the use of an electronic monitor patients can perform spirometry tests at home. The data are stored and can be transferred, giving the care provider the opportunity to look into the data at the health institution. The storage capacity of electronic peak flow meters is of high relevance, as it is proven that peak flow diaries kept by asthmatic children are unreliable. Electronic peak flow meters are preferred if peak flow monitoring is required in children with asthma (27). The effectiveness of telemonitoring has previously been evaluated in many conditions, such as diabetes and hypertension. Nevertheless, there is still a need to obtain evidence related to its clinical effects, cost-effectiveness, impacts on services utilisation, and acceptance by health care providers (28).

\section{Role of the nurse practitioner}

The quality of care has been proven to stay intact when nurses become main care providers in primary care as well as in outpatient care $(29 ; 30)$. With a great deal of autonomy, specialised nurses are able to effectively deliver routine management of chronic conditions, such as asthma. In a nurse-led asthma programme nurses take over the role of the doctor. It has been demonstrated that this can lead to cost-savings, while the effects remain the same. In many cases the nurses will work with an intervention protocol, in which it is stated what autonomic tasks they can perform and when they should consult another physician. The stepwise GINA approach for pharmacological therapy based on asthma severity can serve as the foundation of the intervention protocol. In a telemonitoring programme this would imply that a nurse can monitor and judge individual patient data and take stepwise actions on a daily basis. With regard to patients with less serious complaints a nurse can be the main care provider and physicians have no direct contact with the patient. In these situations the intervention protocol enables the nurse to decrease the treatment by one therapy step (stable asthma) or increase the treatment by one therapy step.

The nurses in a nurse-led telemonitoring programme are sometimes referred to as asthma nurses, respiratory nurses, and specialised nurses and in the Netherlands as nurse practitioners. 


\section{Health Technology Assessment}

Methodology

Medical Technology Assessment (MTA), also referred to as Health Technology Assessment (HTA), is the systematic evaluation of the properties, effects and/or other impacts of health care technology (31). For this purpose, a health care technology is not merely a high tech medical intervention (as can be misinterpreted by using the term MTA) but can be any health procedure, service, or programme within a health care setting.

Figure 1. Graphic illustration of the synthetic character of MTA and the involvement of various research disciplines (Habbema et al.)

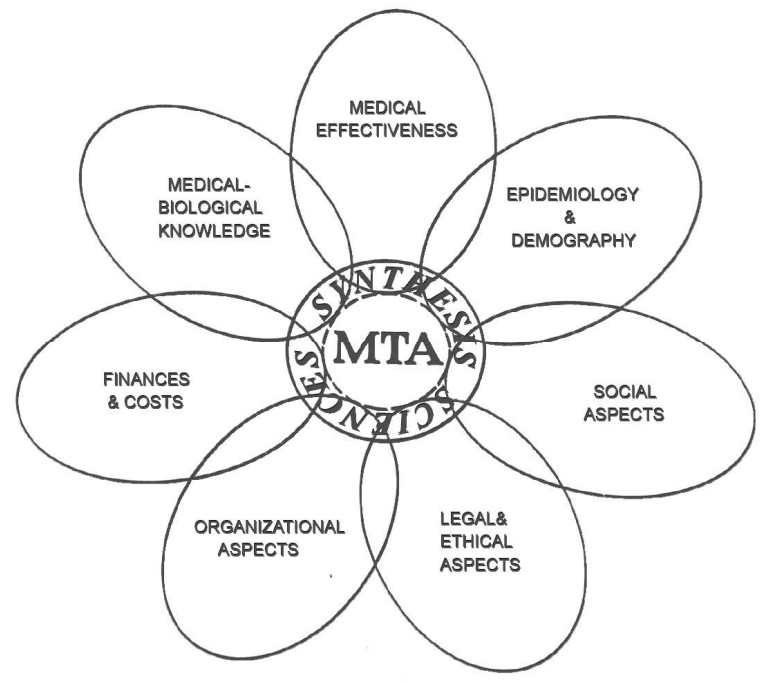

Habbema et al. (32) have developed a graphic illustration to emphasise the synthetic character of HTA. As shown in figure 1, the illustration clarifies the involvement of various research disciplines in HTA. Clinical decision analyses require a synthesis in research aiming to support the decision-making process of the individual patient. The figure shows that not only the effects and the costs of a medical technology intervention are important issues to evaluate with regard to a new medical intervention, but also the process is of high relevance. Consequently, Feeny et al. (33) propose a planning cycle which places health technology assessment in a broad perspective. This Technology Assessment Iterative Loop (TAIL), as shown in figure 2, stresses that assessment is not a single process, but rather a continuing process with modifications applied as knowledge accumulates about the technology. 
Figure 2. Technology Assessment Iterative Loop (Feeny et al.)

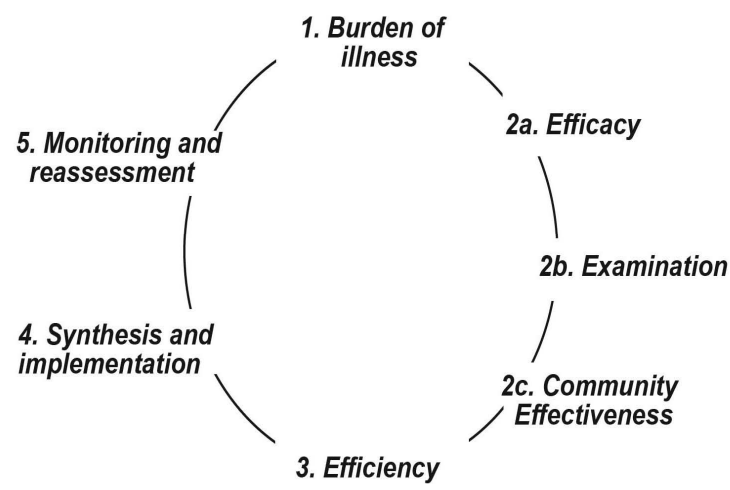

Economic evaluation

In economic evaluations the question of how to obtain the most benefit from the scarce resources available to society is fundamental. In health care, economic evaluations are a branch of HTA which identify, measure, value, and compare costs and outcomes of alternative health care interventions. They deliver useful information for decision making among uses for scarce resources (34). There are several different techniques to perform economic evaluations. In a full economic evaluation both the costs and consequences of at least two alternatives are analysed. In cost analyses only the costs of at least two alternatives are analysed and in efficacy evaluations only the outcomes of at least two alternatives are analysed. In a cost description the costs of only one intervention are analysed and in an outcome description only the outcomes of one alternative are analysed. Furthermore, there are four different methods to measure and value the consequences of health interventions: cost-effectiveness analysis (CEA), cost-utility analysis (CUA), cost-benefit analysis (CBA), and cost-minimisation analysis (CMA). From these four economic evaluations the CEA is the most commonly used. In a CEA the consequences of two or more alternative programmes are measured in natural units and the increments of the indicators are compared. In a cost-utility analysis the consequences are measured in utilities, respectively quality-adjusted-life-years (QALYs) which are a combined measure of quality of life and duration of life. In cost-benefit analyses both the costs and the consequences are expressed in monetary terms. Costminimisation analyses are performed when the assumption is made that the effects of the programmes are equal and are rarely used (35). Another approach to estimate the impact of a new intervention on lifetime resource use, costs, and health outcomes is the 
cost-consequence analysis (34). By listing the costs and consequences the decisionmakers can easily compare alternative options and choose the ones they are interested in.

Furthermore, the viewpoints from which an economic evaluation is performed can differ in studies. This viewpoint can be of significant relevance, as from one point an intervention can look attractive and from another viewpoint unattractive (33). Two common perspectives of an economic evaluation are the societal perspective (all costs and outcomes experienced by all those who are significantly affected by the intervention regardless of who pays or who benefits) and the health care perspective (only health costs and outcomes). In asthma management programmes it is important that health care costs as well as non-health care costs (such as patient expenses) are included in the economic evaluation. This broad viewpoint in an analysis aids the transferability of the results and ensures that the costs of all resource inputs are considered $(35 ; 36)$. Furthermore, the time horizon should be long enough to capture all the differential effects of the options (35).

\section{General objective, research questions and outline of the thesis}

The general objective of this thesis is to investigate the process, health outcomes and cost-effectiveness of a nurse-led telemonitoring programme in asthmatic outpatients.

For this purpose the following research questions were formulated:

What is the quality and what are the outcomes of studies, investigating the costs or costeffectiveness of peak flow monitoring interventions in asthma? (see chapter 2)

How is the process of a nurse-led telemonitoring programme in asthma? (see chapter 3 ) What are the effects of a nurse-led telemonitoring programme in terms of asthma symptoms, asthma-specific quality of life, and medical consumption? (see chapter 4)

What is the use of the multi-attribute instrument, the so called EQ-5D, in children with chronic conditions? (see chapter 5)

What is the cost-effectiveness of a nurse-led telemonitoring programme compared to regular asthmatic outpatient care? (see chapter 6)

In the end, chapter 7 summarises and discusses the main findings, the methodological findings, and the conclusions of all the parts in this thesis. In addition, the generalisation of the study results and the limitations of the study will be discussed. Policy recommendations will be given and suggestions for future research. 


\section{References}

1. GINA Report, Global Strategy for Asthma and Management and Prevention: Revised 2002.

2. Gina Report, Backgrounders: What is asthma?, 2004.

3. World Health Organization (WHO). Bronchial asthma: The scale of the problem. htpp://www.who.int/mediacentre/factsheets/fs206/en/, 2000.

4. Masoli M, Fabian D, Holt S, Beasley R. (2004). Global Initiative for Asthma (GINA): global burden of asthma. http://www.ginasthma.com/Reportltem.asp?|1=2\&/2=2\&intld=94, 2006.

5. Rutten -van Mölken MPMH, Postma MA, Joore MA, van Genugten MLL, Leidl R, Jager JC. Current and future medical costs of asthma and chronic obstructive pulmonary disease in the Netherlands. Respir Med. 1999; 3:281-286.

Barnes PJ, Johnsson B, Klim JB. The costs of asthma. Eur Respir J. 1996; 9:636-642.

6. Hoogendoorn EJI, Feenstra TL, Rutten-van Mölken MPMH: Inventory of the consumption and the costs of care in asthma and COPD in the Netherlands (in Dutch). RIVM rapport 260604001, 2004.

7. Guidelines for the Diagnosis and Management of Asthma' (National Asthma Education and Prevention Program, National Institutes of Health, http://www.aaaai.org/patients/publicedmat/tips/ asthmatriggersandmgmt.stm, 1997.

8. National Heart, Lung and Blood Institute, National Institutes of Health. International consensus report on diagnoses and management of asthma. Betheseda, MD: National Heart, Lung and Blood Institute, National Institutes of Health, 1992 (Publication NO 92.3091).

9. British Thoracic Society, British Paediatric Association, Research Unit of the Royal College of Physicians of London, King's Fund Centre, National Asthma Campaign, Royal College of General Practitioners, et al. Guidelines on the management of asthma. Thorax. 1993; 48 S1-24.

10. Willems D. Tools of care - Explorations into the semiotics of medical technology, Thesis, 1995, 108.

11. Finkelstein J, Manuel R. Cabrera MR, Hripcsak G. Internet-based home asthma telemonitoring - Can patients handle the technology? Chest. 2000; 117:148-155.

12. Goodfellow LT, Rau JL. Devices and adjuncts to self-management for asthma sufferers. Business briefing: long-term health care strategies. www.touchbriefings.com/pdf/14/lth031_p_GOODFE 1.PDF, 2005.

13. Cowie RL, Revitt SG, Underwood MF, et al. The effect of a peak flow-based action plan in the prevention of exacerbations of asthma. Chest. 1997; 112:1534-1538.

Gallefoss F, Bakke PS. Cost-effectiveness of self-management in asthmatics: a 1-yr follow-up randomised, controlled trial. Eur Respir J. 2001; 17:207-213.

14. Kauppinen R, Sintonen H, Vilkka V, Tukiainen H. Long-term (3-year) economic evaluation of intensive patient education for self-management during the first year in new asthmatics. Respir Med. 1999; 93:283-289.

15. Kauppinen R, Vilkka V, Sintonen H, Klaukka T, Tukiainen H. Long-term economic evaluation of intensive patient education during the first treatment year in newly diagnosed adult asthma. Respir Med. 2001; 95:5663.

16. Lindberg M, Ahlner J, Ekström T, Jonsson D, Möller M. Asthma nurse practice improves outcomes and reduces costs in primary health care. Scand J Caring Sci. 2002; 16:73-78.

17. Lucas DO, Zimmer LO, Paul JE, et al. Two-year results from the asthma self-management program: long-term impact on health care services, costs, functional status, and productivity. J Asthma. 2001; 38(4):321-330.

18. McLean W, Gillis J, Waller R. The BC Community Pharmacy Asthma Study: A study of clinical, economic and holistic outcomes influenced by an asthma care protocol provided by specially trained community pharmacists in British Columbia. Can Respir J. 2003; 10(4):195-202.

19. Neri M, Migliori GB, Spanevello A, et al. Economic analysis of two structured treatment and teaching programs on asthma. Allergy. 1996; 51:313-319. 
20. Schermer TR, Thoonen BP, van-den-Boom G, et al. Randomised controlled economic evaluation of asthma self-management in primary health care. Am J Respir Crit Care Med. 2002; 166:1062-1072.

21. Trautner C, Richter B, Berger M. Cost-effectiveness of a structured treatment and teaching programme on asthma. Eur Resp J. 1993; 6:1485-1491.

22. Tschopp JM, Frey JG, Pernet R, et al. Bronchial asthma and self-management education: implementation of Guidelines by an interdisciplinary programme in health network. Swiss Med Wkly. 2002; 132:92-97.

23. Field MJ (ed.). Telemedicine: A guide to Assessing Telecommunications in Health Care. National Academy Press, Washington, 1996.

24. Wootton R. Recent advances: Telemedicine. BMJ. 2001; 323(7312):557-560.

25. Kamps AW, Roorda RJ, Brand PLP. Peak flow diaries in childhood asthma are unreliable. Thorax. 2001; 56(3):180-182.

26. Pare G, Jaana M, Sicotte C. Systemic Review of Home Telemonitoring for Chronic Diseases: The Evidence Base. J Am Med Inform Assoc. 2007; 14(3):269-277.

27. Horrocks S, Anderson E, Salisbury C. Systematic review of whether nurse practitioners working in primary care can provide equivalent care to doctors. BMJ. 2002; 324:819-823.

28. Kamps AWA, Brand PLP, Kimpen JLL, Maillé AR, Overgoor-van de Groes AW, van Helsdingen-Peek LCJAM, Roorda RJ. Outpatient management of childhood asthma by paediatrician or asthma nurse: randomised controlled study with one year follow up. Thorax. 2003; 58:968-973.

29. RBO. Deel 1: Advies Medical Technology Assessment [in Dutch]. Raad voor gezondheidsonderzoek, 1998.

30. Habbema IDF, Casparie AF, Mulder JH, Rutten FFH. Medische Technology Assessment en gezondheidsbeleid. Samson Stafleu: Alphen aan de Rijn, 1989.

31. Feeny D, Guyatt G, Tugwell P. Health care technology: effectiveness, efficiency \& public policy. Montreal: The Institute for Research on Public Policy, 1986.

32. Mauskopf JA, Paul JE, Grant DM, Stergachis A. The role of cost-consequence analysis in healthcare decisionmaking. Pharmacoeconomics. 1998; 13(3):227-288.

33. Drummond MF, Sculpher MJ, Torrance GW, O'Brien BJ, Stoddart GL. Methods for the economic evaluation of health care programmes. 3rd ed. Oxford: Oxford University Press, 2005.

34. Sefton T, Byford S, McDaid D, Hills J, Knapp M. Making the most of it: Economic Evaluation in the social welfare field. Joseph Rowntree Foundation: York Publications, 2002. 
The cost-effectiveness of self-management in asthma a systematic review of peak flow monitoring interventions Int J Technol Assess Health Care 2006, 22:436-442

DCM Willems MA Joore JJE Hendriks EFM Wouters JL Severens 


\section{Chapter 2}

\section{Abstract}

Objectives: It is generally accepted that home peak flow monitoring increases patients' self-management, and could lead to savings. The aim of this review is to analyse costs and the cost-effectiveness of self-management based on peak flow monitoring interventions in asthma.

Methods: A total of 21 studies were included in this review. Data were extracted and methodological and economic quality was assessed. These studies presented economic information regarding self-management interventions based on peak flow monitoring in asthmatics. The mean methodological quality was 4.6 (maximum 8), and the mean economic quality was 12.0 (maximum 15).

Results: In 18 studies the interventions led to net savings compared to usual care, or less intensive intervention. Only three studies found the total costs to be higher in the intervention group. In 13 of the 17 studies which analysed health outcomes, at least one of the reported health outcomes improved statistically significantly after the intervention. However, the methods of economic evaluation differed among the studies, and were not always in line with the standard methodology.

Conclusions: The interventions, costs and outcomes were very diverse. The results emphasise the need for guidelines to increase the comparability of cost-effectiveness evaluations relating to asthma. Only then will it be possible to conclude whether interventions for asthmatics, such as self-management based on peak flow monitoring interventions, are cost-effective. 


\section{Introduction}

Asthma is a chronic inflammatory disorder of the airways which causes recurrent episodes of wheezing, breathlessness, chest tightness, and coughing (34). Around 300 million people worldwide suffer from asthma (24). Despite the continuous improvements in treatment and prevention, and the increase of knowledge resulting from research, its prevalence is increasing in the Western community (24). The human and economic burden associated with asthma is severe (34).

Since asthma is a chronic disorder which cannot be cured, self-management and education are crucial to ensure effective control of asthma. Peak flow monitoring in selfmanagement and education interventions is particularly useful for controlling asthma by demonstrating the variable airflow limitations that characterise the disorder. A peak flow monitor registers airway obstruction by measuring the rate of the peak expiratory flow (PEFR). The PEFR shows the maximum rate of airflow attained during forced vital capacity determination. Increasingly, electronic peak flow monitors with transfer possibilities are replacing standard peak flow monitors, as information communication technology is improving. Self-management including peak flow monitoring seems to increase patient awareness of their disease status and control (12). It also enables substitution of care, possibly saving costs. The effectiveness and cost-effectiveness of self-management and education programs in asthma have been reviewed several times $(6 ; 8-10 ; 14 ; 21 ; 33)$. However, a structured review of costs and the cost-effectiveness of asthma self-management purely based on peak flow monitoring interventions is lacking. The main objective of this review is: "What is the cost-effectiveness of self-management based on peak flow monitoring interventions in asthma?"

\section{Methods}

\section{Literature selection}

The aim of the search strategy was to identify all studies presenting economic information about self-management interventions based on peak flow monitoring in asthmatics. Search engines used to extract relevant studies were: Medline, Pubmed, OHEE-HEED and PsycINFO. Searches were performed up until 1st December 2004, and were restricted to studies in English, German, and Dutch. The search string used was: (('asthma') and ('self-management' or 'monitoring') and ('cost"' or 'economic' or 'effectiveness')).

To be included, peak flow monitoring had to be the main intervention, and separate cost data had to be presented. Studies in which no original empirical outcomes were 


\section{Chapter 2}

presented were excluded. Since only a limited number of studies in this field were full economic evaluations, other types of economic evaluations such as cost-outcome analyses, cost analyses, cost-outcome descriptions, and cost-descriptions (5), were also suitable for inclusion. The search strategy identified 342 potentially relevant articles. Selection took place by reading the abstracts, and, when in doubt, the whole article. Four additional relevant articles were identified from the references of other relevant studies.

The main reason for exclusion was that peak flow monitoring was not the primary intervention (34\%). See table 1 for more details. In the end, 21 studies were included in this review. The collected studies were summarised by DW and checked by MJ using a data extraction form (available on request) based on the CRD Report (3). Methodological and economic quality assessment took place using a checklist from the Cochrane Collaboration (1). Thirteen studies were published in the 1990s and eight were published after the year 2000. Twelve of the articles originated from Europe, seven from the United States, and two from Canada.

Table 1. Overview of inclusion of studies

\begin{tabular}{lcc}
\hline Exclusion criteria & $\begin{array}{c}\text { Number } \\
\text { of studies (\%) }\end{array}$ & $(34)$ \\
\hline Self-management based on peak flow monitoring not as main intervention & 116 & $(25)$ \\
No costs presented & 87 & $(7)$ \\
Review article & 24 \\
No original empirical outcomes presented (other than review articles) & 65 \\
Asthma was not one of the main diseases or no separate data on asthma presented & 33 \\
\hline Total number of studies excluded & 325 \\
Number of studies included & $(19)$ \\
Reference check & 17 \\
Total number of studies included & 4 \\
\hline
\end{tabular}

Interventions

The frequency of peak flow measurements was specified in seven of the 21 studies. The frequency as stated varied from 'regularly' (25), to 'once daily' $(11 ; 19)$, to 'twice daily for one day per week' (27), or 'if symptoms occurred, and at least twice daily in the two weeks before the follow-up visits' (15-17). Self-treatment by medication at home based on peak flow monitoring, was explicitly mentioned in fourteen studies $(2 ; 4 ; 7 ; 11 ; 13 ; 19 ; 20 ; 22 ; 23 ; 25 ; 27 ; 28 ; 30 ; 31)$. In seven studies it was unclear whether selftreatment was applied. In all studies the self-management intervention based on peak flow monitoring also included some form of health education.

In most studies a full detailed description of the content of the intervention was lacking. In four studies $(7 ; 20 ; 28 ; 32)$ the intervention contained a combination of individual 
sessions and group sessions, while in seven studies $(2 ; 4 ; 13 ; 19 ; 22 ; 23 ; 25)$ only individual sessions took place. In nine studies $(11 ; 15-18 ; 23 ; 26 ; 29 ; 31)$ only group sessions took place, and in one study this was unclear (31).

It was notable that in one study the intervention was carried out during hospital admission (30), while in another study sessions were also given at home (28), and in one study during an one-week asthma camp. In Cowie et al. (2) the intervention consisted of one single session (2). Eleven studies described multiple sessions (range 2 to 8 ). The duration of a single session varied from 30 minutes to four hours.

Health outcomes

No health outcomes were described in four studies $(4 ; 13 ; 20 ; 29)$. Clinical outcomes, such as lung function rates, asthma symptoms, and asthma free days were measured in thirteen studies $(2 ; 4 ; 7 ; 15-19 ; 22 ; 25-27 ; 30)$. Lung function parameters most frequently reported included the peak expiratory flow (PEF), the forced expiratory volume in one second (FEV1), and the forced expiratory volume expressed as a percentage of that predicted (FEV\%). Changes in behaviour, knowledge, attitude and compliance were assessed in five studies $(18 ; 22 ; 23 ; 25 ; 32)$. Quality of life was an outcome parameter in 10 studies $(7 ; 15-17 ; 22 ; 23 ; 25 ; 27 ; 28 ; 31)$. The following specific questionnaires were used to measure patient outcomes: the St George's Respiratory Questionnaire (SGRQ) (7;1517), the Asthma Quality of Life Questionnaire (AQLQ) $(25 ; 27 ; 28)$, the Asthma Quality of Life Scale (28), four asthma questions from the Omnibus interviews (7), and the Psychosomatic Discomfort Scale (28). Generic quality of life was measured using the 15D (15-17), the SF-36 (23), the EQ-5D (22), and daily quality of life readings on a fivepoint scale (25).

\section{Direct and indirect costs}

In this study costs have been divided into three categories: direct medical costs, direct non-medical costs, and indirect costs. Direct medical costs such as intervention costs and hospital costs were directly assigned to health care regardless of who was paying. Direct non-medical costs such as time and travel costs were not directly assigned to health care. Indirect costs refer to productivity losses. All the studies described direct medical costs. Data on direct costs were gathered using a variety of measures: telephone interviews, self-administered patient questionnaires, diaries, surveys, medical records, pharmacy reports, claims, and sickness fund records.

However, five studies did not report the total intervention costs as part of the direct medical costs $(4 ; 13 ; 22 ; 25 ; 31)$, while in one study $(32)$ the intervention costs were the 


\section{Chapter 2}

only direct medical costs reported. In Tschopp et al. (31) the only direct medical costs considered were hospitalisation costs. The remaining 19 studies calculated at least two types of direct medical costs, one of which (2) only calculated the intervention costs, and the number of visits to the emergency department with an estimated range of the costs of one visit to the emergency department. In two studies, besides the intervention costs, hospitalisation costs and costs for emergency care were the only direct costs mentioned $(18 ; 20)$. Hospital costs were analysed in 18 of the 21 studies and the cost of emergency care (including urgent medical examinations) in 16 studies. Outpatient visits and/or physician visits were included in 12 studies. Drug costs were calculated in 12 studies.

Six studies misclassified direct non-medical costs as indirect costs (productivity loss). In five studies the time costs of participating in the intervention or seeking health care were incorrectly regarded as indirect costs $(7 ; 11 ; 15-17)$. In another study travel costs to health care facilities and miscellaneous expenses were misclassified as indirect costs (29). A total of 14 studies analysed indirect costs. To measure indirect costs all studies used patient registration such as diaries, questionnaires, and records. In two studies data about sick leave days were retrieved from sickness fund records $(26 ; 30)$.

\section{Quality assessment}

Most of the studies clearly described the inclusion and exclusion criteria and the population, and specified the primary outcome measures. In 12 studies the analysis did not include intention-to-treat analyses $(2 ; 4 ; 7 ; 11 ; 16-18 ; 22 ; 23 ; 25 ; 28 ; 29)$, and in three studies this was unclear.

The study designs were randomised controlled trials $(N=13)$, cohort studies $(N=3)$, and before and after studies $(N=5)$. The mean methodological quality was 4.6 out of a maximum of 15 (sd. 2.0; range 1 to 8 ). Of the 21 studies, 17 studies were full economic evaluations, implying that both the costs and outcomes of at least two alternatives were analysed in these economic evaluations. It was notable that in one study, although it was a full economic evaluation according to the terminology, only intervention costs were included (32). One full economic evaluation was a cost-utility analysis (27) and the 16 remaining full economic evaluations were cost-effectiveness analyses. The remaining four studies were cost analyses, in which only the costs of at least two alternatives were analysed $(4 ; 13 ; 20 ; 29)$.

Common perspectives of an economic evaluation were the societal perspective (all costs and outcomes experienced by all those who are significantly affected by the intervention) and the health care perspective (only health costs and outcomes). Only four studies (all full economic evaluations) mentioned the perspective (all societal) of the economic 
evaluation $(7 ; 26 ; 27 ; 30)$. In addition, two of these studies also adopted the health care perspective $(27 ; 26)$. Among the remaining studies, 10 studies calculated both direct and indirect costs which implies, though not explicitly mentioned, that these studies also adopted a societal perspective. The remaining seven studies only calculated direct medical costs and therefore adopted a health care perspective.

Three studies $(7 ; 15 ; 27)$ calculated an incremental cost-effectiveness ratio (ICER) in line with the standard methodology of economic evaluation (5). Kaupinnen et al. (15) and Gallefoss et al. (7) calculated ICERs for clinical and quality of life outcomes. Schermer et al. (27) presented the incremental costs per QALY gained and successfully treated week gained. In one cost-effectiveness study no ICER was calculated because the groups were equivalent in terms of their health outcomes (17). In the remaining 13 costeffectiveness studies no ICERs were calculated $(2 ; 11 ; 16 ; 18 ; 19 ; 22 ; 23 ; 25 ; 28 ; 31 ; 32)$, or at least not in line with the standard methodology $(26 ; 30)$. The uncertainty of the outcomes was presented in sensitivity analyses in two studies $(7 ; 30)$ while only Schermer et al. (27) presented the uncertainty surrounding the ICER, using bootstrap results of the incremental costs and effects. Furthermore twelve studies did not present measures of variability for the point estimates of primary outcome measures $(2 ; 4 ; 18 ; 20 ; 22 ; 23$; $25 ; 26 ; 28-30 ; 32)$. The heterogeneity of the studies aggravated the transferability of costs. For that reason the costs were only presented in their local currency. The mean score of the quality assessment regarding the economic evaluation was 12.0 out of a maximum of 18 (sd. 2.1; range 9 to 15 ).

\section{Results}

In Gallefoss et al. (7) and Schermer et al. (27) the intervention was dominant compared to regular care. The between-group difference in total costs in Schermer et al. (27) was only statistically significantly lower in the intervention group during the second year. According to the Dutch informal ceiling ratio of $€ 18,000 / Q A L Y$, the probability that the intervention was cost-effective compared to regular care was 52\% (27). In Kauppinnen et al. (15) the cost-effectiveness ratios were all positive which indicates that an extra unit of effect comes at a price.

Of the 18 studies that did not calculate ICERs, only two studies found the total costs to be higher in the intervention group (28;32). However, in Søndergaard et al. (28) the differences were not tested, and in Windsor et al. (32) only the intervention costs were calculated.

Although the symptom plan program in Cowie et al. (2) yielded savings compared to no action plan, the savings from the peak flow based program were even higher. In the 


\section{Chapter 2}

remaining 15 studies the interventions led to net savings compared to usual care or a less intensive intervention $(4 ; 11 ; 13 ; 16-20 ; 22 ; 23 ; 25 ; 26 ; 29-31)$. The statistically significant net savings differed in two studies $(11 ; 19)$, and were not statistically significant in four studies $(16-18 ; 20)$. In the other 9 studies this was unclear.

In 13 of the 17 studies that analysed health outcomes, at least one of the reported health outcomes improved statistically significantly after the intervention $(7 ; 11 ; 15 ; 16 ; 18 ; 19 ; 22$; $23 ; 25 ; 27 ; 30-32)$. In three studies $(16 ; 17 ; 26)$ the difference between groups was not statistically significant, and in one study this was unclear (28). The incremental costs, outcomes and cost-effectiveness ratios are presented in table 2 . If the author stated no statistically significant difference in outcome, the outcome scores were assumed to be zero in this table.

\section{Conclusion}

This review summarised and compared the results of costs and cost-effectiveness of asthma self-management based on peak flow monitoring. A considerable number of eligible studies were found on this subject (21). Nevertheless the interventions studied were very diverse and specific details were often not presented. In addition, asthma quality of life outcomes were measured in only 10 studies, and generic quality of life outcomes in only six studies. Only one study expressed the health outcomes as utilities, and calculated QALYs.

It was noticeable that the studies calculated a variable range of types of costs which made it difficult to compare the total costs of the studies. Because many studies chose to take a limited perspective, not all the relevant costs were included. Furthermore misclassification of non-medical costs appeared in six studies. The methods of economic evaluation differed in many studies, and were not always in line with the standard methodology. Incremental cost-effectiveness ratios were only calculated in three studies. A cost-effectiveness plane, a scatter plot of the uncertainty, and a cost-effectiveness acceptability curve were only presented in the study by Schermer et al. (27). Even after our own calculation of cost-effectiveness ratios from the data presented, it was almost impossible to compare the results, because of the large heterogeneity in study methods. In 13 of the 17 full economic evaluations at least one of the reported health outcomes improved statistically significantly after the intervention. These related especially to clinical symptoms. Of the 17 full economic evaluations the intervention (or more intensive intervention) was dominant in no less than 14 studies, four of which described the difference as statistically significant. In Windsor et al. (32), Kaupinnen et al. (15) and Søndergaard et al. (28) an extra unit of effect came at a price. In all four cost-analyses 
the intervention led to net savings. Given the results of the studies the use of selfmanagement programs based on peak flow monitoring in asthmatics seems to be cost effective or even cost saving. However, one should bear in mind that these results were obtained in trial settings, and may be an overestimation of cost-effectiveness in day-today practice. Besides this, the relatively poor methodological quality of the studies and the difference in methods mean that the conclusions of this review should be interpreted with caution. First, the methodological quality of cost and cost-effectiveness studies in asthmatics should be improved. Secondly, uniform guidelines for economic evaluations of asthmatics are needed to compare cost-effectiveness evaluations of self-management based on peak flow monitoring interventions. Moreover, until the societal perspective is applied in economic evaluations, resulting in a cost-utility analysis, it is almost impossible to fully synthesise the evidence of self-management based on peak flow monitoring interventions. As a result, based on the available evidence reviewed in this study, we can not conclude whether self-management based on peak flow monitoring interventions in asthmatics is cost-effective. 


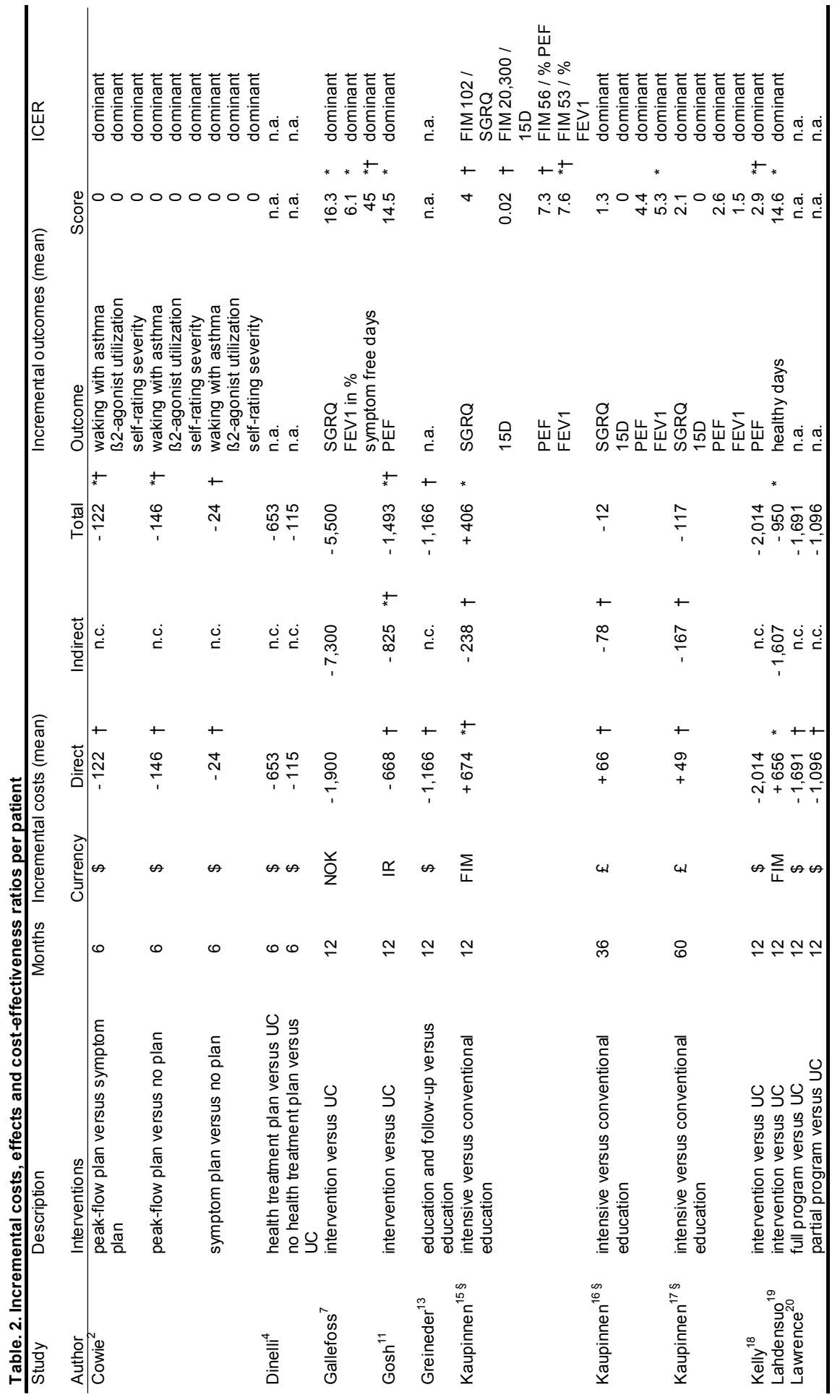




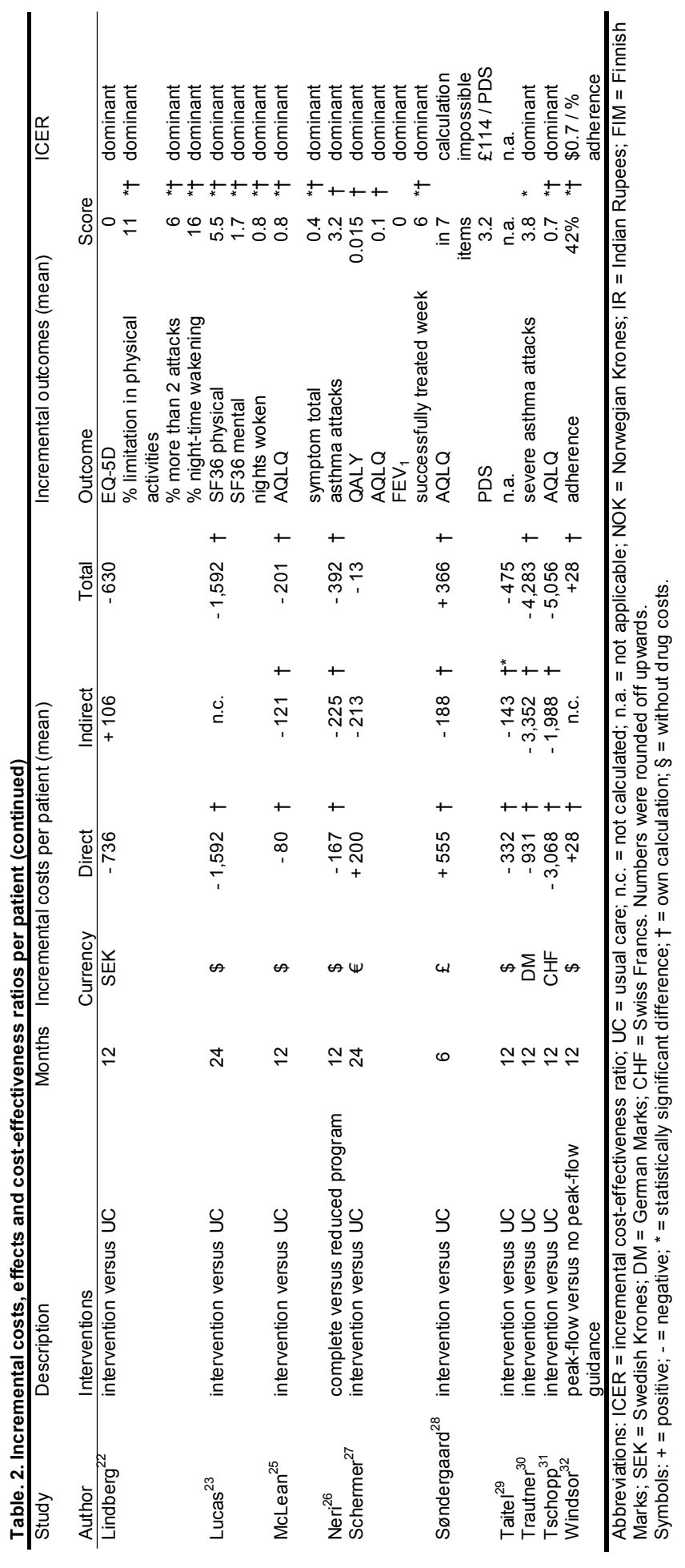




\section{Chapter 2}

\section{References}

1. Ament A, Birch S, Brown $P$, et al. Criteria list for conducting systematic reviews based on economic evaluation studies. In: Donaldson C, Mugford M, Vale L, eds. Evidence-based health economics: from effectiveness to efficiency in systematic review. London: BMJ-Books; 2002:99-114.

2. Cowie RL, Revitt SG, Underwood MF, et al. The effect of a peak flow-based action plan in the prevention of exacerbations of asthma. Chest. 1997;112:1534-1538.

3. CRD Report Number 4 (2nd Edition). Undertaking Systematic Reviews of Research on Effectiveness. University of York. March 2001.

4. Dinelli DL, Higgins JC. Case management of asthma for family practice patients: a pilot study. Mil Med. 2002;67:231-234.

5. Drummond MF, O'Brien B, Stoddart GL, Torrance GW. Methods for the economic evaluation of health care programmes (2nd Edition). Oxford: Oxford University Press; 1997;1-51.

6. Feenstra TL, Rutten-van Mölken MPMH, Jager JC, Van Essen-Sandvliet LEM. Cost effectiveness of guideline advice for children with asthma: a literature review. Pediatr Pulmonol. 2002;34(6): 442-454.

7. Gallefoss F, Bakke PS. Cost-effectiveness of self-management in asthmatics: a 1-yr follow-up randomised, controlled trial. Eur Respir J. 2001;17:207-213.

8. Gibson PG, Powell H, Coughlan J, et al. Self-management education and regular practitioner review for adults with asthma (Cochrane Review). Cochrane Library, Issue 3. Published Online First: 22 July 2002. doi: 10.1002/14651858.CD001117

9. Gibson PG, Powell H, Coughlan J, et al. Limited (information only) patient education programs for adults with asthma (Cochrane Review). Cochrane Library, Issue 3. Published Online First: 21 January 2002. doi: 10.1002/14651858.CD001005

10. Gibson PG, Ram FSF, Powell H. Asthma education. Respir Med. 2003;97:1036-1044.

11. Ghosh CS, Ravindran P, Joshi M, Stearns SC. Reductions in hospital use from self-management training for chronic asthmatics. Soc Sci Med. 1998;46:1087-1093.

12. Goodfellow LT, Rau JL. Devices and adjuncts to self-management for asthma sufferers. Business briefing: long-term health care strategies. www.touchbriefings.com/pdf/14/lth031_p_GOODFE 1.PDF. Published Online First: 2003. Date last accessed: October 262005.

13. Greineder DK, Loane KC, Parks P. A randomised controlled trial of a pediatric asthma outreach program. J Allergy Clin Immunol. 1999;103(3):436-440.

14. Guevara JP, Wolf FM, Grum CM, Clark NM, Cates CJ. Effects of educational interventions for selfmanagement of asthma in children and adolescents: systematic review and meta-analysis. $\mathrm{Br} \mathrm{Med} \mathrm{J}$. 2003;326(7402):1308-1309.

15. Kauppinen R, Sintonen $\mathrm{H}$, Tukiainen $\mathrm{H}$. One-year economic evaluation of intensive vs conventional patient education and supervision for self-management of new asthmatic patients. Respir Med. 1998;92:300-307.

16. Kauppinen R, Sintonen H, Vilkka V, Tukiainen H. Long-term (3-year) economic evaluation of intensive patient education for self-management during the first year in new asthmatics. Respir Med. 1999;93:283-89.

17. Kauppinen R, Vilkka V, Sintonen H, Klaukka T, Tukiainen H. Long-term economic evaluation of intensive patient education during the first treatment year in newly diagnosed adult asthma. Resp Med. 2001;95:56-63.

18. Kelly CS, Shield SW, Gowen CL, et al. Outcomes analysis of a summer asthma camp. J Asthma. 1998;35(2):165-171.

19. Lahdensuo A, Haahtela T, Herrala J, et al. Randomised comparison of cost effectiveness of guided selfmanagement and traditional treatment of asthma in Finland. Br Med J. 1998;316:1138-1139.

20. Lawrence G. Asthma self-management programs can reduce the need for hospital-based asthma care. Respir Care. 1995;40(1):39-43.

21. Liljas B, Lahdensuo A. Is asthma self-management cost effective? Patient Educ Couns. 1997;32:S97-S104. 


\section{A systematic review of peak flow monitoring interventions}

22. Lindberg M, Ahlner J, Ekström T, Jonsson D, Möller M. Asthma nurse practice improves outcomes and reduces costs in primary health care. Scand J Caring Sci. 2002;16:73-78.

23. Lucas DO, Zimmer LO, Paul JE, et al. Two-year results from the asthma self-management program: long-term impact on health care services, costs, functional status, and productivity. J Asthma. 2001;38(4):321-330.

24. Masoli M, Fabian D, Holt S, Beasley R. Global Initiative for Asthma (GINA): global burden of asthma. www.ginasthma.com/Reportltem.asp?|1=2\&l2=2\&intld=94 Published Online First: May 2004. Date last accessed: October 262005.

25. McLean W, Gillis J, Waller R. The BC Community Pharmacy Asthma Study: A study of clinical, economic and holistic outcomes influenced by an asthma care protocol provided by specially trained community pharmacists in British Columbia. Can Respir J. 2003;10(4):195-202.

26. Neri M, Migliori GB, Spanevello A, et al. Economic analysis of two structured treatment and teaching programs on asthma. Allergy. 1996;51:313-319.

27. Schermer TR, Thoonen BP, van-den-Boom G, et al. Randomised controlled economic evaluation of asthma self-management in primary health care. Am J Respir Crit Care Med. 2002;166:1062-1072.

28. Søndergaard B, Davidson F, Kirkeby B, Rasmussen M, Hey H. The economics of an intensive education programme for asthmatic patients. Pharmacoeconomics. 1992;1(3):207-212.

29. Taitel MS, Kotses H, Bernstein IL, Bernstein DI, Creer TL. A self-management program for adult asthma. Part II: Cost-benefit analysis. J Allergy Clin Immunol. 1995;95(5):672-676.

30. Trautner C, Richter B, Berger M. Cost-effectiveness of a structured treatment and teaching programme on asthma. Eur Resp J. $1993 ; 6: 1485-1491$.

31. Tschopp JM, Frey JG, Pernet R, et al. Bronchial asthma and self-management education: implementation of Guidelines by an interdisciplinary programme in health network. Swiss Med Wkly. 2002;132:92-97.

32. Windsor RA, Bailey WC, Richards JM, et al. Evaluation of the efficacy and cost effectiveness of health education methods to increase medication adherence among adults with asthma. Am J Public Health. 1990;80(12):1519-1521.

33. Wolf FM, Guevara JP, Grum CM, et al. Review: self management education improves outcomes in children and adolescents with asthma. Evid Based Med. 2003;8(4):114-115.

34. World Health Organization (WHO). Bronchial asthma: The scale of the problem. www.who.int/mediacentre/ factsheets/fs206/en/ Date last update: January 2000. Date last accessed: October 262005. 
The process evaluation of a nurse-led telemonitoring programme for asthmatics

Based on: J Telemed Telecare 2007; 13(6):310-317(8)

DCM Willems

MA Joore

JJE Hendriks

$\mathrm{RAH}$ van Duurling

EFM Wouters

JL Severens

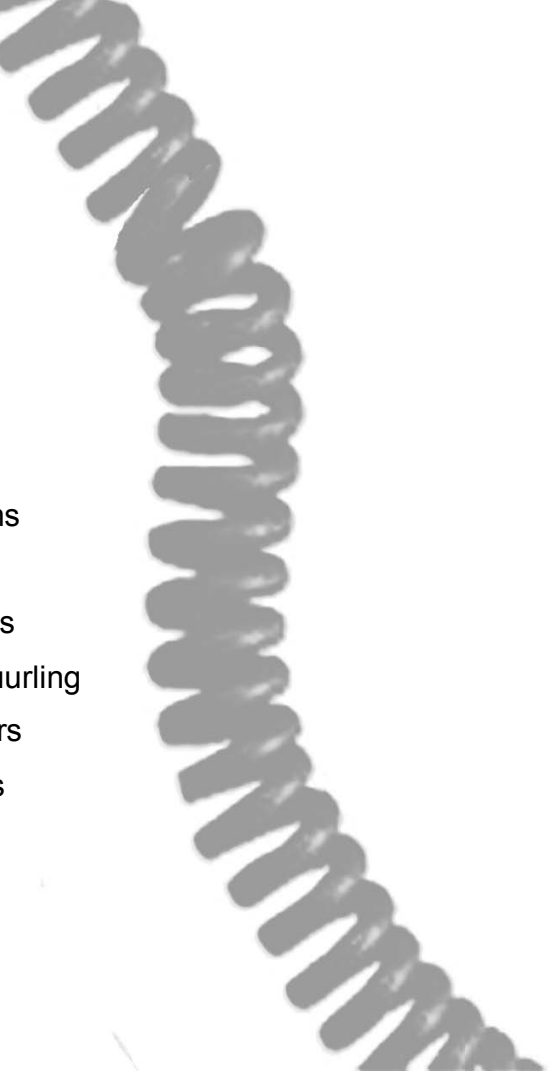




\section{Chapter 3}

\section{Abstract}

The purpose of this study was to perform a process evaluation of a nurse-led telemonitoring programme for asthmatics. The indicators used to evaluate the programme included programme feasibility, consistency of peak expiratory flow (PEF) tests, compliance, intervention and study protocols, and patient satisfaction.

The study followed 109 asthmatic outpatients (56 children, 53 adults) for 12 months. Patients were randomly assigned to the intervention group or the regular outpatient care group. A nurse studied the home monitoring data for the intervention group and took stepwise actions in accordance with the intervention protocol.

The acceptance to participate was $39 \%$. Although technical and logistical problems did occur, the dropout rate was low. At least $75 \%$ of the PEF manoeuvres were valid for two third of 20 patients. Compliance with the study protocol was high. The actual number of recorded PEF tests averaged less than the two tests per day that were required by the protocol. Patient satisfaction was high and, after one year, less than $20 \%$ of the participants chose to discontinue their participation.

The results indicate that if a nurse-led telemonitoring programme is introduced into a motivated group of mild to moderate asthmatics, the programme is feasible, reliable, and satisfying. 


\section{Introduction}

Globally, asthma is one of the most common chronic illnesses and the prevalence of asthma is still increasing (1). Asthma can be treated and controlled under the guidance of health care professionals (2) and patient self-management is an important component in this control.

Patient self-monitoring is crucial to asthma self-management (3). It enables patients to monitor and evaluate their asthma symptoms at home. Increasingly, information and communication technologies are being used in health care. Collectively, these activities are referred to as 'e-health'. Telemedicine is a form of e-health that encompasses many different activities involving the use of telecommunication technologies in health care. Telemedicine has opened up a wide range of possibilities for health care providers to monitor patients at a distance (4). As the use of telemedicine activities increased, the role of nurses also expanded $(5 ; 6)$. Respiratory nurses (in this study also referred to as nurse practitioners) have become frequent health care providers in the treatment and control of asthma. These two developments come together in nurse-led telemonitoring programmes. Lung function tests, from which the peak expiratory flow (PEF) is the most commonly used, are recorded in an electronic monitor. By transferring monitor data to a central database, nurse practitioners are able to continuously supervise the disease status of individual patients.

Yellowlees (2005) emphasised that information regarding the development of telemedicine programmes must be shared. It is important that the programmes are properly evaluated and that the results are published regardless of whether they are positive or negative. Process evaluations concentrate on the conditions necessary to reach the desired effect. These evaluations can provide cohesive information on the application of a programme within the target population. They help explain the success or lack of effect of a programme and can thereby enable others to replicate the programme and generate improvements. In a controlled study, a process evaluation can be used to determine how the participants experienced the activities, whether they encountered difficulties and if participants were satisfied with the intervention programme (8). In this study the process evaluation of a nurse-led telemonitoring programme for asthmatics is described. The indicators for the process evaluation were the feasibility of the intervention, the consistency of the PEF tests, compliance with both the study and intervention protocols, and patient satisfaction with the telemonitoring programme. 


\section{Chapter 3}

\section{Methods}

\section{Study design}

This study concerns the process evaluation of a nurse-led telemonitoring programme for asthmatics. The effectiveness of the programme was evaluated in a single centre, prospective randomised controlled trial at the University Hospital Maastricht in the Netherlands. The nurse practitioner used a list of random numbers to allocate the patients to one of the two treatment arms. The control group received regular outpatient care. The duration of follow-up was one year and the measurements took place at baseline, 4, 8, and 12 months.

The process evaluation was performed alongside the randomised controlled trial and this paper focuses on the intervention group. The study protocol was approved by the Medical Ethical Committee of the University Hospital Maastricht in the Netherlands.

\section{Study population}

Hospital outpatients 7 years of age and older with an asthma severity of stage I - III, as described in the GINA guidelines, were considered eligible for the nurse-led telemonitoring programme. Included in the study were patients who were considered competent enough to use an asthma monitor and possessed a household phone connection. Excluded from the study were patients with respiratory co-morbidity (such as cystic fibrosis or congenital lung abnormalities) since these patients frequently require outpatient visits.

The study population was recruited from the Medical Respiratory Department and the Department of Paediatrics of the University Hospital Maastricht. The inclusion and exclusion criteria were determined by verifying patient records. Potential eligible patients received an information letter by post. The letter was accompanied by an informed consent form and provided the respondent with an opportunity to indicate the reason for declining to participate in the study. If informed consent was obtained, an appointment was scheduled with the nurse practitioner for the baseline visit at the hospital.

\section{Intervention}

A nurse practitioner was the main care provider for the patients in the intervention group. The patients received an asthma monitor for home use. The monitor was a portable hand-held device with a corresponding modem on which spirometry test results, symptoms, medication use, and events could be registered. The accuracy of the spirometry measurements of the asthma monitor was proven to be very good (9). 
Patients were able to review their spirometry test results on the monitor screen. The transfer of the monitor data to the nurse practitioner's computer was performed by connecting the modem to the household phone connection.

During the baseline visit, the nurse practitioner explained how the monitor can be used and the patients were asked to demonstrate the correct use of the monitor. The patients were requested to perform daily PEF tests - one in the morning and one in the evening. For each measurement, the best of three consecutive PEF tests was stored in the monitor. If their asthma symptoms increased, they were asked to perform additional PEF tests. Before the end of the first visit, the patient was asked to demonstrate the correct use of the monitor. The nurse practitioner studied the patient data daily and took stepwise actions in accordance with the intervention protocol. This protocol was based on the GINA guidelines and the guidelines of the Dutch College of General Practitioners $(2 ; 10 ; 11)$. The protocol described stable asthma as patients who have minimal symptoms (only cough at night or after exercise), sporadic mild exacerbations, and no emergency room visits. Also, stable asthmatics were expected to experience no limitations in activities and physical exercise and no absence from school/work due to asthma. In addition, stable patients were expected to use less than three puffs per week of a b2-mimetic and were expected to encounter only minimal adverse events from the medication. The PEF value had to be 80 to $100 \%$ of the patient's personal best PEF. The protocols definition of an exacerbation was PEF value $<80 \%$ of normal value, asthma symptoms and use of bronchodilators. A severe exacerbation was defined as a PEF value $<50 \%$ of normal value, with little or no reversibility after bronchodilators. The appendix contains a more detailed description of the intervention protocol.

Indicators

The following indicators for the process evaluation of the intervention were defined and monitored: the feasibility of the intervention, the consistency of PEF tests on the asthma monitor, patient compliance with the study and intervention protocols, and patient satisfaction.

Descriptive statistics are presented for all indicators (percentages, means, standard deviations, medians). All data analyses were conducted using SPSS-pc, version 12.0.1.

\section{Feasibility of the intervention}

The feasibility of the intervention was indicated by the patient recruitment. Feasibility was also assessed by means of technical and logistic problems. If a planned transmission did not take place, the nurse practitioner should according to the protocol, contact the patient 


\section{Chapter 3}

during working hours. Technical and logistical problems were continuously registered in a data file maintained by the nurse practitioner. Technical problems included transmission or monitor failures. Logistical problems included the patient accessibility in the case of missing transfer data and the accessibility of the nurse practitioner when patients had complaints or symptoms.

\section{Consistency of PEF tests}

Before the study was initiated, it was decided that forced expiratory volumes at one second (FEV1) would not to be used in the analyses, due to the lack of reliability of the total forced vital capacity (FVC) manoeuvres of most patients. Spirometry data could be stored and adequately evaluated even after several weeks/months, since the memory of the monitor was sufficient enough. To judge the validity of the PEF manoeuvre the nurse practitioner looked for the gradient of the flow volume curve up to the PEF value and for the fluency in the decline during FEF75-25VC. If $75 \%$ of the curves of one patient had the same steepness and adequate decrease, the PEF value was accepted as valid. To assess the validity of the visual curves of the software system, two respiratory assistants independently judged the consistency of the PEF technique of 10 adults and 10 children during the first nine months of the study. As the PEF value varies $+/-10 \%$, some manoeuvres would not be maximal. In this case, the respiratory assistant estimated the time delay and the angle of incline for the first part to PEF from the curve as objective judgement of the PEF manoeuvre. It was not possible to compute the data from the software, as only five flow values per manoeuvre were stored. For that reason, only visually the steepness between the separate measurements of each patient were performed.

\section{Patient compliance with the study and the intervention protocol}

The study protocol included questionnaires and diaries to measure the effectiveness of the programme in terms of clinical symptoms, quality of life, and use of health care. The compliance on these questionnaires and diaries was assessed. During the baseline visit, the first questionnaire was collected and the first diary was handed out. All subsequent questionnaires and diaries were sent by mail every 3 months. If these questionnaires and diaries were not returned on time, patients received a telephone call as a reminder. Patients were asked to report clinical asthma symptoms and health care use in their diaries daily for the duration of one month. Along with each measurement, the participants were asked to complete quality of life questionnaires (EQ-5D (12), SF36 (13), AQLQ(14), PAQLQ $(15 ; 16)$ and TACQOL(17)). If available, the children received 
the paediatric versions. Children aged 12-18 were asked to complete the questionnaires on their own. The parents or caregivers received a proxy version of the questionnaire for children between the ages of 7-12 (16).

Key determinants of patient compliance with the intervention protocol were the twicedaily PEF tests on the monitor and the monthly transfers of the monitor data. The PEF test results were stored on the monitor and the transfer data was registered in the data file of the nurse practitioner.

\section{Patient satisfaction}

A questionnaire was developed for the intervention group to measure patient satisfaction with the programme. This questionnaire was partly based on the satisfaction questionnaire developed by Finkelstein et al. (2000). After 4 and 12 months the satisfaction questionnaires were mailed to the patients. The first questionnaire consisted of 21 items and the second questionnaire of 19 items. The items in both questionnaires can be divided into three clusters: application, study protocol, and self-management and satisfaction. In addition, the first questionnaire included two items about the written and verbal information regarding the use of the monitor that were provided by the nurse practitioner during the baseline visit. All items had four response options. There was an adult version (18 years and older), an adolescent version (12 to 18 years) and a proxy version ( 7 to 12 years). After 12 months participation, the patients were asked to return their monitor to the nurse practitioner at the hospital. During this visit, the patient was asked five open questions regarding the monitor use. The questions concerned the patients' experiences with the monitor, whether it was easy or difficult to use, what they liked or disliked, and how they felt about returning the monitor.

\section{Results}

\section{Study population}

Between January 2003 and January 2004, 55 patients were allocated to the intervention group (26 adults and 29 children). The mean age of the adults was 46 years and the children's mean age was 11 . Of the adults, $42 \%$ were male. Of the children, $72 \%$ were male. On average, the severity of asthma, classified according to the GINA classification, was mild persistent for the adults and moderate persistent for the children. Lung function values demonstrated that the entire intervention group had reversible asthma. In total, 5 of the 55 patients were lost to follow-up in the intervention group due to the patients' refusal to continue their participation (two adults and three children). Four cases were lost to follow-up immediately after the baseline measurement and one case occurred 


\section{Chapter 3}

after the third measurement at eight months. See figure 1 for a flowchart of the study progress.

Figure 1. Flowchart of study progress

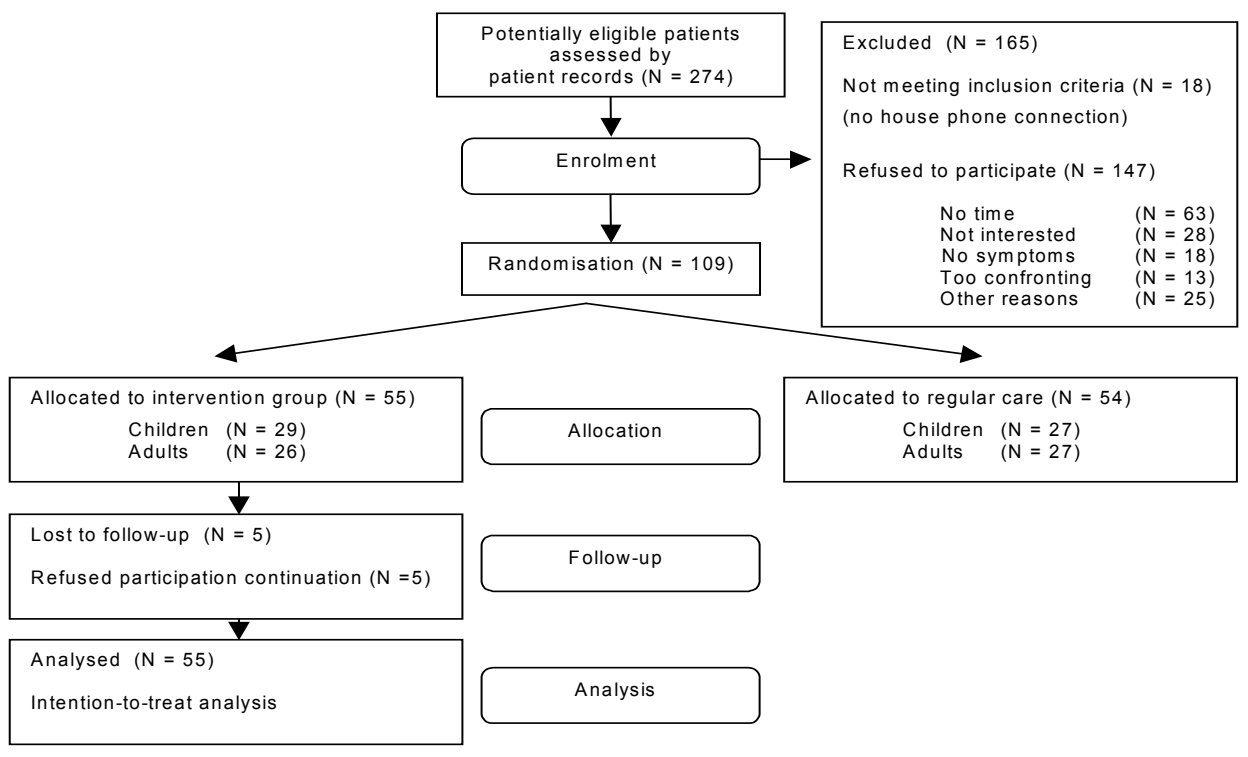

Feasibility

A total of 274 potentially eligible outpatients were identified and approached by mail. Of the total group, 18 patients were excluded due to the lack of a house phone connection (7\%) and 147 patients refused participation in the study (54\%). The most frequently reported reasons for refusing to participate were: 'having no time' $(43 \%)$, 'being uninterested' (19\%), 'not experiencing asthma symptoms' (12\%), and 'finding participation too confronting' ( $9 \%$ ). Consequently, $40 \%$ of the potential participants were included in the study.

Technical problems were registered 19 times (33\%) by 18 patients during the study year (eight adults and ten children). These technical problems consisted of 16 transmission or monitor failures related to the malfunction of the device. On one occasion, the technical problem was related to the software and, on two occasions, the problem was due to the house phone connection. The nurse practitioner made seven home visits due to technical problems and three patients visited the nurse practitioner at the hospital. In total, four modems (three children and one adult) and five monitors (one child and four adult) were replaced during the follow-up year. 
One logistic difficulty was the nurse practitioner's inability to contact the patients by the telephone. In the one-year follow-up, 136 planned data transfers were missed. In one fifth of the missed data transfers ( 29 of 136 ), the nurse practitioner was unable to contact the patients via telephone. This occurred more often among the adults $(26 \%)$ than among the children (14\%). Another logistic barrier was that the nurse practitioner could only be reached during working hours. The patients who experienced symptoms outside working hours (weekends and evenings) were forced to contact other care providers.

The consistency of PEF data

Of the two independent respiratory assistants who judged the consistency of the PEF technique, the first assistant accepted the PEF data for seven of the ten adults and seven of the ten children as being valid. The second respiratory assistant determined that the PEF data were valid for six adults and six children. This indicates that, for two thirds of these 20 patients, at least $75 \%$ of the PEF manoeuvres were considered valid. Based on the results of the received data during the study, the nurse practitioner undertook 39 actions (such as medication adjustment and spirometry instructions) among the adults and 78 actions among the children.

\section{Compliance}

Compliance with the study protocol (questionnaires and diaries) was high. At baseline, $100 \%$ compliance for filling in the questionnaires was achieved because patients who did not bring completed questionnaires to the baseline visit were asked to complete the questionnaire during this visit. The telephone reminders for diaries or questionnaires that were not returned on time also increased compliance. After reminders, the response on the measurements varied from $85 \%$ to $92 \%$ with regard to the questionnaires and from $81 \%$ to $90 \%$ with regard to the diaries. No differences between adults and children were observed.

In table 1 , the average number of PEF tests a day are analysed for the total group (intention-to-treat) and the total group minus the patients who were lost at follow-up. According to the protocol, PEF testing should have been performed at least twice daily.

The average number of PEF tests a day was lower than the two tests that were required by the protocol. Of the total group, an average of 1.53 tests was performed daily. When excluding the drop outs from the analysis, the average number of tests increased to 1.67 a day. On average, the adults performed more tests than the children (1.78 and 1.31 including drop outs; compared to 1.90 and 1.46 excluding drop outs, respectively). Of the calculated average, seven adults performed two or more PEF tests a day and only two 


\section{Chapter 3}

children performed two or more PEF tests a day. In both analyses, the majority of the participants performed less then two PEF tests a day.

Table 1. PEF tests a day after 12 months

\begin{tabular}{lccccccccccccc}
\hline & \multicolumn{1}{c}{ Adults } & \multicolumn{1}{c}{ Children } & \multicolumn{1}{c}{ Total } \\
\hline Intention-to-treat & & & & & & & & & & & \\
PEF tests a day & $\mathrm{N}$ & mean & $(\mathrm{sd})$ & median & $\mathrm{N}$ & mean & $(\mathrm{sd})$ & median & $\mathrm{N}$ & mean & (sd) & median \\
$\geq 2$ (per protocol) & 7 & 3.28 & 1.55 & 2.20 & 2 & 2.05 & 0.23 & 2.05 & 9 & 3.00 & 1.44 & 2.07 \\
$<2$ (not per protocol) & 19 & 1.22 & 0.60 & 1.30 & 27 & 1.26 & 0.60 & 1.45 & 46 & 1.24 & 0.60 & 1.39 \\
Total & 26 & 1.78 & 1.30 & 1.64 & 29 & 1.31 & 0.62 & 1.47 & 55 & 1.53 & 1.02 & 1.48 \\
Without drop outs & & & & & & & & & & & & \\
PEF tests a day & $\mathrm{N}$ & mean & $(\mathrm{sd})$ & median & $\mathrm{N}$ & mean & $(\mathrm{sd})$ & median & $\mathrm{N}$ & mean & $(\mathrm{sd})$ & median \\
$\geq 2$ (per protocol) & 7 & 3.28 & 1.54 & 2.20 & 2 & 2.05 & 0.23 & 2.05 & 9 & 3.01 & 1.44 & 2.07 \\
$<2$ (not per protocol) & 17 & 1.34 & 0.51 & 1.30 & 24 & 1.41 & 0.44 & 1.47 & 41 & 1.38 & 0.47 & 1.45 \\
Total & 24 & 1.90 & 1.27 & 1.72 & 26 & 1.46 & 0.46 & 1.50 & 50 & 1.67 & 0.96 & 1.59 \\
\hline
\end{tabular}

The monitor enabled patients to register their asthma symptoms (coughing, wheezing and shortness of breath) through the use of buttons. Patients only registered symptoms, if they considered this feasible. When patients did enter their symptoms, a clear relationship between the increase of symptoms and the decrease of PEF was shown.

As described earlier, the patients were asked to transfer their monitor data once a month and more often if they were experiencing symptoms. Taking into account the five dropouts in the intervention group, the results show that, after 12 months, 600 planned transfers should have taken place ( 288 transfers by 24 adults and 312 transfers by 26 children). The nurse practitioner registered that 80 of the planned transfers from the adults $(28 \%)$ were missed and 56 planned transfers from the children $(18 \%)$. If the nurse practitioner tried to contact the patients who missed a planned transfer by telephone, 28 of the expected transfers from the adults (10\%) did not take place and 9 transfers from the children $(3 \%)$.

\section{Patient satisfaction}

No important differences between the first satisfaction questionnaire at 4 months and the second questionnaire at 12 months were found. The results of the satisfaction questionnaire at 12 months are presented in table $2 \mathrm{a}$ and $2 \mathrm{~b}$. The results indicate that most participants did not experience the use of the monitor as difficult. Overall, a minority of the patients $(4 \%)$ experienced a significant number of symptoms. Almost half of the patients found it slightly difficult to register the symptoms on the monitor using the buttons but no differences between the adults and children were found. The maximum 
amount of PEF tests that was acceptable was on average two measurements a day. The majority of the patients (87\%) found playing an active role in their asthma management an improvement. Of all the patients, $65 \%$ felt 'slightly' to 'significantly safer' while using the monitor. With regard to the care provider the patients are more outspoken. Of the participants, $84 \%$ did not feel less safe at all contacting the nurse instead of the doctor. Half of the patients found the telephone contacts with the nurse just as pleasant as doctor visits. Most patients appreciated it 'much' (40\%) or even 'very much' (54\%) that the nurse immediately noticed lung function deterioration. When participants were asked if they wanted to continue to use the monitor, the most frequent answer was 'maybe' for the children and 'certainly' for the adults. In the final month, 'not so many symptoms' to 'no symptoms' were experienced by $59 \%$ of the patients.

During the last study visit, participants were interviewed regarding the use of the monitor. The questions were answered by 20 of the 26 adults and by 22 of the 29 children. Of the 42 respondents, 28 were very positive about the monitor use and 13 explicitly mentioned that they appreciated the contact with the nurse practitioner. The advantage most often mentioned was the demonstration of the PEF test results (25 patients). Seven patients mentioned that it was hard to remember to use the monitor every day. The majority of the patients (32) found it easy to operate the monitor, although ten patients found it difficult to register the symptoms on the monitor using the buttons. Eleven patients (six children and five adults) were relieved that they could return the monitor after the study year, while another 11 patients (three children and eight adults) wanted to continue using the monitor.

\section{Conclusion}

This study described the process evaluation of a nurse-led telemonitoring programme for asthmatics. Indicators for the process evaluation were the feasibility of the intervention, the consistency of the PEF tests, patient compliance with the study protocol and the intervention protocol, and patient satisfaction with the programme. The feasibility was determined by recruitment, drop out, and technical and logistic problems. Overall, the recruitment was difficult; the acceptance rate was only $39 \%$. However, once participating, the drop outs were low (9\%). Overall, 33\% of the participants experienced technical problems, for the most part, related to malfunctioning devices. Of the devices (monitor and modem), 10\% had to be replaced. Logistical problems occurred when the nurse practitioner had to contact the patients regarding missed data transfers. In one fifth of the cases, the nurse practitioner was unable to reach the patients by telephone. 


\section{Chapter 3}

Analyses of the subset of the data revealed that with regard to the consistency of the PEF, in two thirds of the patients, at least $75 \%$ of the PEF manoeuvres were valid.

The compliance with the study protocol was very high during each of the four measurements. Overall, the compliance was above $80 \%$ after reminders.

Compliance with the intervention protocol was lower. The average number of PEF tests a day was lower than the two tests that were required by the protocol. However, on average, 1.53 tests were performed a day. The satisfaction was good with regard to the education, application of the monitor, study protocol, and self-management. After one year, less than $20 \%$ of the participants did not want to continue with the programme. With regard to the feasibility of the programme there were patient barriers, as the willingness to participate was low (39\%). Probably the research setting had some influence on this. Furthermore, the use of more advanced telemonitors would have prevented patient exclusion due to the lack of a house phone connection. Recently, telemonitors have been developed which can transfer data by house phones as well as by Bluetooth. A minority of the patients who refused to participate found participating too confronting $(9 \%)$. For this group, monitoring would be almost impossible. Noncompliance could be a rational choice on the part of the patient. These patients may have found the use of the monitor and the guidance of a nurse practitioner to be more problematic than their asthma symptoms. This was possibly, at least partly, due to the fact that the patients had only mild to moderate asthma. A limitation of the spirometry test was the restriction to using PEF tests only. While FEV1 could be measured and stored in the monitor, due to the lack of reliability of the total FVC manoeuvre by most patients (especially in younger children), these were not suitable.

On average, compliance with the questionnaires and diaries was very high. This implies that, although a considerable amount of patients were unwilling to participate, the patients that did participate were motivated. This corresponds with the low dropout rate and with the fact that dropouts mainly occurred directly after baseline. Without telephone reminders by the nurse practitioner $23 \%$ of the planned data transfers did not take place and on average patients did not perform two or more PEF tests a day. The fact that compliance with the study protocol was less good could be partially attributed to the experienced burden. For most patients, the maximum number of measurements they found acceptable was two measurements a day. From this, it can be concluded that the maximum accepted level of burden had been reached. Lack of compliance with the protocol may also be attributable to the fact that most patients experienced relatively few symptoms. Also, patients indicated that they found it difficult to remember to monitor 
Table 2a. Satisfaction questionnaire

\begin{tabular}{|c|c|c|c|c|}
\hline \multicolumn{2}{|c|}{ Items } & Children & Adults & Total group \\
\hline \multicolumn{5}{|c|}{ Education (only in questionnaire administered at 4 months): } \\
\hline \multirow[t]{5}{*}{1} & $\begin{array}{l}\text { Did you get all the necessary written information about the use of } \\
\text { the monitor from the nurse during the baseline visit? }\end{array}$ & $\mathrm{N}=26$ & $\mathrm{~N}=23$ & $N=49$ \\
\hline & very limited & $4 \%$ & $0 \%$ & $2 \%$ \\
\hline & partial & $8 \%$ & $0 \%$ & $4 \%$ \\
\hline & almost all & $23 \%$ & $13 \%$ & $18 \%$ \\
\hline & all & $65 \%$ & $87 \%$ & $75 \%$ \\
\hline \multirow[t]{6}{*}{2} & Did you get all the necessary verbal information about the use of & & & \\
\hline & the monitor from the nurse during the baseline visit? & $\mathrm{N}=26$ & $\mathrm{~N}=24$ & $\mathrm{~N}=50$ \\
\hline & very limited & $0 \%$ & $0 \%$ & $0 \%$ \\
\hline & partial & $4 \%$ & $0 \%$ & $2 \%$ \\
\hline & almost all & $12 \%$ & $8 \%$ & $10 \%$ \\
\hline & all & $85 \%$ & $92 \%$ & $88 \%$ \\
\hline \multicolumn{5}{|c|}{ Application: } \\
\hline \multirow[t]{5}{*}{1} & How complicated is the use of the monitor? & $\mathrm{N}=24$ & $\mathrm{~N}=20$ & $\mathrm{~N}=44$ \\
\hline & very & $0 \%$ & $0 \%$ & $0 \%$ \\
\hline & moderately & $0 \%$ & $0 \%$ & $0 \%$ \\
\hline & slightly & $33 \%$ & $20 \%$ & $27 \%$ \\
\hline & not at all & $67 \%$ & $80 \%$ & $73 \%$ \\
\hline \multirow[t]{5}{*}{2} & How difficult is it to perform the spirometry test? & $\mathrm{N}=24$ & $\mathrm{~N}=21$ & $\mathrm{~N}=45$ \\
\hline & very & $0 \%$ & $0 \%$ & $0 \%$ \\
\hline & moderately & $0 \%$ & $0 \%$ & $0 \%$ \\
\hline & slightly & $8 \%$ & $19 \%$ & $13 \%$ \\
\hline & not at all & $92 \%$ & $81 \%$ & $87 \%$ \\
\hline \multirow[t]{5}{*}{3} & How difficult is registering complaints on the monitor? & $N=24$ & $N=20$ & $N=44$ \\
\hline & very & $0 \%$ & $5 \%$ & $2 \%$ \\
\hline & moderately & $4 \%$ & $10 \%$ & $7 \%$ \\
\hline & slightly & $58 \%$ & $35 \%$ & $48 \%$ \\
\hline & not at all & $38 \%$ & $50 \%$ & $43 \%$ \\
\hline \multirow[t]{5}{*}{4} & How difficult is it transferring data by modem? & $\mathrm{N}=24$ & $\mathrm{~N}=21$ & $\mathrm{~N}=45$ \\
\hline & very & $0 \%$ & $0 \%$ & $0 \%$ \\
\hline & moderately & $0 \%$ & $0 \%$ & $0 \%$ \\
\hline & slightly & $4 \%$ & $5 \%$ & $4 \%$ \\
\hline & not at all & $96 \%$ & $95 \%$ & $96 \%$ \\
\hline \multirow[t]{6}{*}{5} & How much of your time does the use of the monitor take in the & & & \\
\hline & morning? & $\mathrm{N}=24$ & $N=21$ & $\mathrm{~N}=45$ \\
\hline & very significant amount & $0 \%$ & $0 \%$ & $0 \%$ \\
\hline & considerable & $4 \%$ & $0 \%$ & $2 \%$ \\
\hline & little & $42 \%$ & $48 \%$ & $44 \%$ \\
\hline & very little & $54 \%$ & $52 \%$ & $53 \%$ \\
\hline \multirow[t]{2}{*}{6} & How many minutes does the use of the monitor on average take? & & & \\
\hline & ........ mean minutes (range) & $\begin{array}{l}1.8 \\
(0.1-5.0)\end{array}$ & $\begin{array}{l}4.0 \\
(0.1-22.5)\end{array}$ & $\begin{array}{l}2.9 \\
(0.1-25.5)\end{array}$ \\
\hline \multirow[t]{7}{*}{7} & What is the maximum frequency of spirometry tests per day you & & & \\
\hline & think you can tolerate? & $\mathrm{N}=24$ & $\mathrm{~N}=20$ & $\mathrm{~N}=44$ \\
\hline & morning & $13 \%$ & $15 \%$ & $14 \%$ \\
\hline & morning and evening & $83 \%$ & $70 \%$ & $75 \%$ \\
\hline & morning, afternoon and evening & $4 \%$ & $15 \%$ & $9 \%$ \\
\hline & morning, twice in the afternoon and in the evening & $0 \%$ & $0 \%$ & $0 \%$ \\
\hline & Study protocol: & & & \\
\hline \multirow[t]{6}{*}{8} & How difficult is answering diary questions? & $\mathrm{N}=24$ & $\mathrm{~N}=21$ & $\mathrm{~N}=45$ \\
\hline & very & $0 \%$ & $0 \%$ & $0 \%$ \\
\hline & moderately & $0 \%$ & $10 \%$ & $4 \%$ \\
\hline & slightly & $29 \%$ & $29 \%$ & $29 \%$ \\
\hline & not at all & $71 \%$ & $62 \%$ & $67 \%$ \\
\hline & Self-management and satisfaction: & & & \\
\hline \multirow[t]{5}{*}{1} & How important are the spirometry test results for you? & $\mathrm{N}=24$ & $\mathrm{~N}=21$ & $\mathrm{~N}=45$ \\
\hline & not & $8 \%$ & $5 \%$ & $7 \%$ \\
\hline & somewhat & $17 \%$ & $19 \%$ & $18 \%$ \\
\hline & very & $50 \%$ & $48 \%$ & $49 \%$ \\
\hline & extremely & $25 \%$ & $29 \%$ & $27 \%$ \\
\hline
\end{tabular}


Table 2b. Satisfaction questionnaire

\begin{tabular}{|c|c|c|c|c|}
\hline \multicolumn{2}{|c|}{ Items } & Children & Adults & Total group \\
\hline & Self-management and satisfaction: & & & \\
\hline \multirow[t]{5}{*}{2} & $\begin{array}{l}\text { Do you think it is an improvement that you now play an active } \\
\text { role in your asthma management? }\end{array}$ & $N=24$ & $\mathrm{~N}=20$ & $\mathrm{~N}=44$ \\
\hline & certainly not & $0 \%$ & $5 \%$ & $2 \%$ \\
\hline & little & $8 \%$ & $15 \%$ & $11 \%$ \\
\hline & yes & $54 \%$ & $35 \%$ & $46 \%$ \\
\hline & certainly & $38 \%$ & $45 \%$ & $41 \%$ \\
\hline \multirow[t]{5}{*}{3} & $\begin{array}{l}\text { How important is it for you to know that your spirometry results } \\
\text { can be reviewed in the hospital by the nurse immediately after } \\
\text { the test? }\end{array}$ & $\mathrm{N}=24$ & $\mathrm{~N}=21$ & $\mathrm{~N}=45$ \\
\hline & not at all & $8 \%$ & $19 \%$ & $13 \%$ \\
\hline & somewhat & $21 \%$ & $10 \%$ & $16 \%$ \\
\hline & very & $58 \%$ & $52 \%$ & $56 \%$ \\
\hline & extremely & $13 \%$ & $19 \%$ & $16 \%$ \\
\hline \multirow[t]{5}{*}{4} & Does the use of the monitor interfere with your daily activities? & $\mathrm{N}=24$ & $\mathrm{~N}=21$ & $\mathrm{~N}=45$ \\
\hline & a great deal & $0 \%$ & $0 \%$ & $0 \%$ \\
\hline & very little & $4 \%$ & $5 \%$ & $4 \%$ \\
\hline & little & $4 \%$ & $19 \%$ & $11 \%$ \\
\hline & no & $92 \%$ & $76 \%$ & $84 \%$ \\
\hline \multirow[t]{5}{*}{5} & Do you feel safer while using the monitor? & $\mathrm{N}=20$ & $\mathrm{~N}=23$ & $\mathrm{~N}=43$ \\
\hline & not at all & $35 \%$ & $50 \%$ & $42 \%$ \\
\hline & slightly & $17 \%$ & $15 \%$ & $16 \%$ \\
\hline & moderately & $44 \%$ & $25 \%$ & $35 \%$ \\
\hline & significantly & $4 \%$ & $10 \%$ & $7 \%$ \\
\hline \multirow[t]{6}{*}{6} & Do you feel less safe contacting the nurse instead of the & & & \\
\hline & doctor? & $\mathrm{N}=24$ & $\mathrm{~N}=21$ & $\mathrm{~N}=45$ \\
\hline & significantly & $0 \%$ & $0 \%$ & $0 \%$ \\
\hline & moderately & $4 \%$ & $5 \%$ & $4 \%$ \\
\hline & slightly & $17 \%$ & $5 \%$ & $11 \%$ \\
\hline & not at all & $79 \%$ & $91 \%$ & $84 \%$ \\
\hline \multirow[t]{6}{*}{7} & Do you think having telephone contacts with the nurse instead & & & \\
\hline & of doctor visits is pleasant? & $\mathrm{N}=24$ & $\mathrm{~N}=21$ & $\mathrm{~N}=45$ \\
\hline & not & $4 \%$ & $5 \%$ & $4 \%$ \\
\hline & no difference & $46 \%$ & $52 \%$ & $49 \%$ \\
\hline & pleasant & $42 \%$ & $19 \%$ & $31 \%$ \\
\hline & very & $8 \%$ & $24 \%$ & $16 \%$ \\
\hline \multirow[t]{6}{*}{8} & Do you appreciate it that lung function deterioration is & & & \\
\hline & immediately noticed by the nurse? & $\mathrm{N}=23$ & $\mathrm{~N}=20$ & $N=43$ \\
\hline & no & $0 \%$ & $0 \%$ & $0 \%$ \\
\hline & somewhat & $9 \%$ & $5 \%$ & $7 \%$ \\
\hline & much & $39 \%$ & $40 \%$ & $40 \%$ \\
\hline & very much & $52 \%$ & $55 \%$ & $54 \%$ \\
\hline \multirow[t]{5}{*}{9} & Would you like to use the monitor in the future? & $\mathrm{N}=24$ & $\mathrm{~N}=21$ & $\mathrm{~N}=45$ \\
\hline & no & $4 \%$ & $14 \%$ & $9 \%$ \\
\hline & unlikely & $17 \%$ & $14 \%$ & $16 \%$ \\
\hline & maybe & $42 \%$ & $29 \%$ & $36 \%$ \\
\hline & certainly yes & $38 \%$ & $43 \%$ & $40 \%$ \\
\hline \multirow[t]{5}{*}{10} & How many symptoms did you experience the last months? & $\mathrm{N}=24$ & $\mathrm{~N}=21$ & $\mathrm{~N}=45$ \\
\hline & a lot & $0 \%$ & $10 \%$ & $4 \%$ \\
\hline & not so many & $63 \%$ & $57 \%$ & $60 \%$ \\
\hline & almost none & $29 \%$ & $19 \%$ & $24 \%$ \\
\hline & no & $8 \%$ & $14 \%$ & $11 \%$ \\
\hline \multirow[t]{6}{*}{11} & If you had no symptoms: if you would develop more symptoms & & & \\
\hline & in the future, would you then like to continue the monitor use? & $\mathrm{N}=2$ & $\mathrm{~N}=2$ & $\mathrm{~N}=4$ \\
\hline & no & $50 \%$ & $0 \%$ & $25 \%$ \\
\hline & unlikely & $0 \%$ & $0 \%$ & $0 \%$ \\
\hline & maybe & $50 \%$ & $50 \%$ & $50 \%$ \\
\hline & certainly yes & $0 \%$ & $50 \%$ & $25 \%$ \\
\hline
\end{tabular}

Numbers were rounded off upwards. 
daily. The use of some sort of reminders, such as an alarm installed in the monitor device, may help patients to be more compliant. On average, the patients did not find the application of the monitor complicated at all. Most found it easy to operate the monitor. This corresponds with the results reported by Finkelstein et al. (2000) in which a spirometer and a palmtop computer were used for self-testing at home. Ten patients found it difficult to register the complaints on the monitor using the buttons. This is probably because the time to register symptoms on the monitor is relatively short.

In a study by Adams et al. (2006), the authors stated that in comparison to care provided by a physician, a nurse specialist delivers equivalent follow-up care for asthma patients discharged from the hospital as a result of acute asthma. An important finding of our study was that $84 \%$ of the patients felt not at all less safe contacting the nurse instead of the doctor. This is in contrast to a study by van Baar et al. (2006) in which patients expressed concerns about the limitations of telecommunication contacts compared to face-to-face encounters and were worried that clinicians would not be in a position to perform physical examinations over the telephone.

In this study, education was only provided during the intake visit and was mainly restricted to information regarding the use of the monitor and the intervention protocol. Specific information about asthma was not given and no group activities were undertaken. The importance of education in self-management programmes is often emphasised. If the focus is on patient empowerment and stimulation of selfmanagement, more extensive asthma education is recommended.

The findings of this study emphasise that a telemonitoring programme is not feasible for all asthmatics. The programmes should be offered to patients who are motivated because patients themselves are the decision makers in their self-management.

From the present study it can be concluded that there are technical and logistical barriers to overcome regarding nurse-led self-management interventions. However, if the nurseled telemonitoring programme is introduced to a motivated group of patients with mild to moderate asthma, the programme will be feasible and reliable and patient satisfaction will be high. 


\section{Chapter 3}

\section{References}

1. See http://www.who.int/mediacentre/factsheets/fs206/en/. Last checked 26 October 2006

2. See htpp//www.ginasthma.com/Reportltem.asp?|1=2\&l2=2\&intld=94. Last checked 26 October 2006

3. See htpp//www.touchbriefings.com/pd/14/th013_p_GOODFE 1.PDF. Last checked 26 October 2006

4. Vlaskamp FJ, Webers K, Peters-Volleberg GW, van Halteren AR. Telemedicine and telecare in home-care: historical developments and expectations for the future. Rijksinstituut voor Volksgezondheid en Milieu (RIVM rapport) 2001:1-54 (in Dutch)

5. Hobbs R, Murray ET. Specialist liaison nurses, Brit Med J 1999;318(7185):683-684

6. Vrijhoef HJ, Diederiks JP, Spreeuwenberg C, Wolffenbuttel BH, van Wilderen LJ. The nurse specialist as main care-provider for patients with type 2 diabetes in a primary care setting: effects on patient outcomes. Int $\mathrm{J}$ Nurs Stud 2002;39(4):441-51

7. Yellowlees PM, Succesfully developing a telemedicine system. J Telemed Telecare 2005;11(7):331-335

8. Hulscher ME, Laurant MG, Grol RP. Process evaluation on quality improvements interventions. Qual Saf Health Care 2003;12:40-46

9. Richter K, Kanniess F, Mark B, Jörres RA, Magnussen H. Assessment of accuracy and applicability of a new electronic peak flow meter and asthma monitor. Eur Respir J 1998;12:457-462

10. Hendriks, J. J. E., Wesseling, G. J., Van Duurling, R. A. H. , \& Wouters, E. F. M. Werkprotocol: PRISMAstudie [Patient research in self-management of asthma]. 2001

11. Dirksen WJ, Geijer RM, De Haan M, De Koning G, Flikweert S, Kolnaar BG. NHG-Standaard Astma bij kinderen [Guidelines of the Dutch College of General Practitioners: asthma in children]. Huisarts Wet 1998;41(3):130-143

12. EuroQol Group. Euroqol - a new facility for the measurement of health related quality of life. Health Policy 1990;16:199-208

13. Brazier J, The SF-36 health survey - a tool for economists. Health Econ 1993;2(3):213-215

14. Juniper EF, Guyatt GH, Ferrie PJ, Griffith LE. Measuring quality of life in asthma. Am Rev Respir Dis 1993;147(4):832-838

15. Juniper EF, Guyatt GH, Feeny DH, Ferrie PJ, Griffith LE, Townsend M. Measuring quality of life in children with asthma. Qual Life Res 1996;5(1):35-46

16. Juniper EF, Guyatt GH, Feeny DH, Ferrie PJ, Griffith LE, Townsend M. Measuring quality of life in the parents of children with asthma. Qual Life Res 1996;5(1):27-34

17. Verrips, GH, Vogels TG, Koopman HM, et al Health-Related Quality of Life Measure for Children - the TACQOL. J Appl Therapeut 1998;1/4:357-60

18. Finkelstein J, Cabrera M, Hripcsak G. Internet-based Home Asthma Telemonitoring: Can patients handle the technology? Chest 2000;117:148-155

19. Adams RJ, Smith BJ, Ruffin RE. Patient preferences for autonomy in decision making in asthma management. Thorax 2001;56:126-132

20. Van Baar JD, Joosten $\mathrm{H}$, Car J, et al Understanding reasons for asthma outpatient (non)-attendance and exploring the role of telephone and e-consulting in facilitating access to care: exploratory qualitative study. Qual Saf Health Care 2006;15:191-195 


\section{Appendix}

\section{The intervention protocol}

The care providers of patients who were younger than 18 years of age were also involved in the intervention. They were the contact persons who, if necessary, assisted the children in the use of the monitor and maintained contact with the nurse practitioner. Patients were asked to transfer the first data one week after the baseline visit. If no problems occurred, the patients were asked to transfer their data every month during the first week of the month. In the second week, the nurse practitioner checked to ensure that all data had been received and evaluated the data. If the data were not received within the predetermined time span or the data were not normal for that patient, the nurse practitioner contacted the patient by telephone. In the case of stable asthma, no therapeutic intervention was considered necessary.

Outpatient appointments were made at the patient's request if transfers failed, or if the nurse practitioner noticed incorrect use of the device and/or bad expiration technique.

In the case of an acute and severe asthma exacerbation, the patient was instructed to contact their general physician at once.

In the case of a less severe exacerbation, as experienced by the patient, the patient was instructed to contact the nurse practitioner by phone and connect the monitor to the modem. If the nurse practitioner was inaccessible they could contact their general practitioner. In both cases the patient was asked to perform extra measurements and send the data immediately. When the nurse practitioner was contacted, the patient was instructed to increase the asthma medication. The nurse practitioner determined if the exacerbation was a sporadic incident or more structural and then, following the protocol, advised the patient to increase the treatment by one therapy step. In the case of an incident, the patient was asked to transfer the data one week later. If medication use was increased, the patient was asked to collect and transfer the data one week later and again two weeks later.

If the standard treatment of an exacerbation was not successful, the nurse practitioner contacted the treating physician to decide whether or not a steroid course was necessary. In adults this involved consulting a lung specialist and in children a paediatric lung specialist. The nurse practitioner provided the physician with all the available data and arranged the prescriptions. The physician had no direct contact with the patient during this process. If the peak expiratory flow test results and symptoms did not improve after several hours, the nurse practitioner advised the patient by telephone to consult the treating physician at the hospital. If the treating physician determined that the 


\section{Chapter 3}

exacerbation was serious, the patient was admitted to the hospital. The treating physician decided whether to increase the dosage of the medication, and after discharge, the patient was asked to transfer the data weekly for one month. If the symptoms disappeared, the patient was instructed to submit the data at the beginning of each month, as was the case before admission to the hospital.

\section{Assessment of the received data and three monthly treatment evaluations}

The nurse practitioner compared the singular flow curves to the baseline PEF tests to validate the PEF tests. The PEF curve was used as the first reference. The changes in PEF curves were evaluated by the steepness of the curve and the distance between the top of the curve (= PEF) and the y-axis. If the PEF curve was not comparable with the intake curve, the patient was encouraged to improve his or her effort. If, after several trials, the technique was still incorrect, the patient was asked to meet with the nurse practitioner.

According to the protocol, every three months, the nurse practitioner could adjust the medication one step up or one step down. If the peak PEF curve was within normal range and the symptoms were minimal, the nurse practitioner asked the patient to decrease the treatment level by one step. If a patient was uncomfortable with this, the nurse practitioner attempted to reach a consensus with the patient. 
Chapter 4

The effectiveness of nurse-led telemonitoring of asthma $\mathrm{J}$ Eval Clin Pract; in press

DCM Willems MA Joore JJE Hendriks FHM Nieman

JL Severens

EFM Wouters 


\section{Chapter 4}

\section{Abstract}

Background and objective: The aim of the study was to evaluate the effects on, and the relationship between, asthma symptoms, asthma-specific quality of life, and medical consumption of a nurse-led telemonitoring intervention compared to regular care in asthma in the Netherlands.

Methods: One hundred and nine asthmatic outpatients (56 children; 53 adults) were randomly assigned to the treatment arms (12 months follow-up). The control group received regular outpatient care, while the intervention group used an asthma monitor with modem at home with an asthma nurse as the main care provider. Clinical asthma symptoms and medical consumption were measured by using diaries. Asthma-specific quality of life was measured by the (Paediatric) Asthma Quality of Life Questionnaire.

Results: The study population generally represented mild to moderate asthmatics. The results show improvement in follow-up, but no statistical significant differences between the groups were observed. Moderate to high correlations were found within the outcome parameters, but the most remarkable was the low, and statistically significant, correlation between asthma-specific quality of life (daily functioning) and the self-reported beta-2 agonists (medicines used to relieve an asthma attack).

Conclusion: Overall, the telemonitoring programme evaluated in this study did not significantly decrease asthma symptoms or medical consumption, or improve asthmaspecific quality of life. The results showed that a telemonitoring programme on its own is not a guarantee of success. The patients' perception of asthma-specific quality of life (daily functioning) should be a key element in asthma telemonitoring programmes. 


\section{Introduction}

Asthma is a chronic disease that affects the airways and is characterised by recurrent attacks of breathlessness and wheezing. About 300 million people suffer from asthma worldwide (1). An essential element in the management of asthma is the continuous monitoring of asthma severity in order to be able to take steps to prevent worsening of asthma and to manage attacks. Therefore, home monitoring of asthma has a central role in asthma self-management programs. Advances in information and communication technology have created the potential to monitor the asthma status of patients at a distance using telemonitoring. These telemonitoring applications have the advantage that outside help and support is offered to patients to maintain self-monitoring. Furthermore, the time to initiate treatment can be reduced. This study is a randomised controlled trial to investigate the effectiveness of a nurse-led telemonitoring programme based on peak flow measurements in asthmatic outpatients.

Lung function monitoring in self-management and education interventions is useful for controlling asthma by providing an objective measure of respiration. A peak flow monitor registers airway obstruction by measuring the rate of the peak expiratory flow (PEFR). The PEFR shows the maximum rate of airflow attained during forced vital capacity determination. Increasingly, electronic peak flow monitors with transfer possibilities are replacing standard peak flow monitors, as information communication technology is improving. In electronic asthma monitors, the data is stored in the monitor and can be supervised at a distance after the data is transferred by modem to a central database. The storage and transfer ability of the electronic monitor can be very helpful, since some studies warn of non-adherence during self-monitoring or misinterpretation of the lung function tests by the patients themselves (2-4). As concluded in the review by Horrocks et al. (5), the role of supervisor can be performed by specialised nurses, since this leads to similar effects as when supervision is performed by doctors.

Self-management including peak flow monitoring seems to increase patient awareness of their asthma and control (6). As a result, these interventions are known to improve asthma status $(7 ; 8)$. Because no valid gold standard of asthma status exists, these interventions are often evaluated using a range of outcome measures (9]. Mostly, objective measures of lung function are supplemented by subjective measures of symptoms, health care utilisation, functioning and quality of life. The use of multiple outcomes may however lead to ambiguous results. Understanding the relationship between measures of asthma status may facilitate the interpretation of research findings and more parsimonious evaluation strategies. 


\section{Chapter 4}

The primary objective of this study was to investigate the effects of a nurse-led telemonitoring programme based on peak flow measurements on asthma symptoms (coughing, production of sputum, and shortness of breath or wheezing), medical consumption (health care utilisation and self-reported medication use), and asthmaspecific quality of life. Furthermore, the secondary objective was to increase the understanding of the relationship between measures of asthma status, also the relationships between these measures were investigated.

\section{Methods}

\section{Study design}

The study design was a single centre prospective randomised controlled trial. Randomisation took place on patient level after stratification by age (ages 7 to 18 versus 18 years and older), since regular care differs between these age groups. The asthma nurse used a list of random numbers to allocate the patients to one of the two treatment arms. The duration of follow-up was 12 months, and measurements were performed at baseline, 4, 8, and 12 months.

\section{Study population}

The study population consisted of asthmatic outpatients from the Medical Respiratory Department and the Department of Paediatrics. The patients aged 7 and older with an asthma severity of stage I-III as described in the Global Initiative For Asthma (GINA) guidelines were potentially eligible (10). Furthermore, the patients had to be competent to use an asthma monitor, and had to possess a household phone connection. Exclusion criteria were severe co-morbidity (such as COPD or heart failure), and structural defects in the upper airways or lungs. The power calculation for this study was based on improvement in asthma-specific quality of life, with a minimal important difference of 0.5 points on the 7-point overall scale in the Asthma Quality of Life Questionnaire (AQLQ) or the Paediatric Asthma Quality of Life Questionnaire (PAQLQ) (11-13).

One-sided testing, a power of $80 \%$, and a significance level of 0.05 with a drop out percentage of $10 \%$ resulted in a minimal inclusion of 51 patients in the intervention group and 51 patients in the control group.

\section{Intervention and study protocol}

The control group received regular outpatient care at the University Hospital Maastricht in the Netherlands. In case of stable asthma these patients received three to six monthly medical check ups by their lung specialist (18 years and older) at the Medical 
Respiratory Department or paediatrician (ages 7 to 18) at the Department of Paediatrics. In case of exacerbations the patients received additional treatment as usual in the Netherlands.

The intervention group used an electronic asthma monitor at home, and had an asthma nurse (also referred to in the Netherlands as the nurse practitioner) as the main care provider. The monitor was a portable hand-held device with a matching modem. It was possible to register lung function values and symptoms on the monitor. The asthma nurse educated the patients about the telemonitoring protocol, lung function values and the asthma monitor during the baseline visit. Furthermore, the asthma nurse gave instructions on the use of the monitor and the contacts with the asthma nurse. In this study the patients were asked to perform daily peak flow measurements both in the morning and in the evening and more often if they were having complaints. Patients were asked to transfer the monitor data every month and more frequently if they were having asthma complaints. The transfer was performed by connecting the modem to the household phone connection. Monitor data were transferred to the computer of the asthma nurse. The asthma nurse studied the data daily during working hours and classified the asthma following a stepwise intervention protocol. The protocol was based on the GINA guidelines and the Dutch College of General Practitioners (10;14-16). According to this protocol the asthma nurse was allowed to decrease (after 3 months of stable asthma) or increase asthma medication by one step. A physician was only consulted if necessary. Following this procedure, the asthma nurse adjusted or maintained the treatment. In most cases this was possible by telephone contact. This approach meant that the patients could be continuously monitored and treatment immediately adjusted whenever indicated. The caregivers of patients aged younger than 18 years were also involved in the intervention. If necessary, they assisted the children in the monitor use and the contacts with the asthma nurse.

\section{Research questions}

Self-management programmes for asthmatics appear to improve the health outcomes for asthmatics and reduce the amount of medical consumption (17-20).

In this study the primary research question was: 'What are the effects of a nurse-led telemonitoring intervention on clinical asthma symptoms, asthma-specific quality of life, and medical consumption compared to regular care?' We expected that the intervention would lead to a decrease of the clinical symptoms, an improvement in asthma-specific quality of life, and a decrease in care consumption (except for the asthma nurse). 


\section{Chapter 4}

Medication use was expected to possibly go both ways (increase at the start, decrease after asthma control).

The second research question was: 'Which relationships are there between and among lung function values, symptom scores, asthma medication, and domains of asthmaspecific quality of life?' Strong correlations were expected to be present among lung function values (PEF and FEV1), symptom scores (morning and evening coughing, production of sputum, and shortness of breath or wheezing), asthma medication (corticosteroids and beta-2 agonists), and dimensions of asthma-specific quality of life. Based on previous studies we expected low correlations between lung function values and self-reported symptoms, and lung function values and asthma-specific quality of life (11-13). Moderate relationships were expected between symptoms and asthma-specific quality of life (11). Little is known about the correlations between self-reported asthma medication and other patient outcomes. Furthermore, contradicting literature exists on the relation between self-reported medication and asthma-specific quality of life, so no assumptions were made $(21 ; 22)$.

\section{Outcome parameters}

The primary outcome parameter in the study was asthma-specific quality of life. Secondary outcome parameters were clinical asthma symptoms (lung function values and self-reported symptoms), and medical consumption. Asthma-specific quality of life was measured in both groups by the AQLQ in adults, and the PAQLQ in children (1113). The AQLQ contains 32 items with a score range from 1 to 7 , and four domains: Symptoms, Activity Limitation, Emotional Function, and Environment. The PAQLQ contains 23 items with a range from 1 to 7 , and domains: Symptoms, Activity limitation, and Emotional Function. Children aged 12-18 years were asked to complete the adolescent version of the questionnaire by themselves (12). For children aged 7-12 years, the parents or caregivers received a proxy version of the questionnaire (13). Lung function values concerned the PEF and the FEV1 which were registered at the start and end of the study. Clinical asthma symptoms were coughing, production of sputum, and shortness of breath / wheezing in the morning and the evening. Patients in both groups registered these symptoms in diaries (range of 0 to 3 ) along with their asthma-related medical consumption (four times for the duration of one month). Medical consumption which was registered in the diaries, consisted of self-reported medication use, visits or telephone contacts with the general practitioner (GP) and GP-assistant, consultation with the nurse practitioner at the GP, lung specialist, paediatrician, asthma nurse, and emergency room (ER) visits. Medication was divided in beta-2 agonists, corticosteroids, 
combined beta-2 agonists and corticosteroids medication, and ointments or nose sprays with corticosteroids. Beta-2 agonists are 'relievers', medicines that help to relief asthma attacks by quickly opening the narrowed airways. Most commonly these medicines are taken by an inhaler. Corticosteroids are 'preventers', anti-inflammatory medicines that reduce the inflammation in the airways if taken regularly and are effective for treatment of bronchial inflammation. Corticosteroids are available in ointments, nose sprays, inhalers, pills and by injection. During the baseline visit the first questionnaire was collected and the first diary was handed out after obtaining informed consent. From then on, all questionnaires and diaries were sent by mail every 3 months to all the participants (control and intervention group). If these questionnaires and diaries were not returned on time, the patients received a telephone call as a reminder.

\section{Statistics}

Analyses to measure the effects of the nurse-led telemonitoring intervention were performed on an intention-to-treat basis. Based on the observed scores on equivalent items of the AQLQ and PAQLQ, combined scale constructions were carried out for the domains Symptoms, Activity limitation, and Emotional Function. The domain Environmental stimulus was excluded from the analysis, since this domain only exists in the AQLQ, and not in the PAQLQ. Scale construction was therefore not carried out for the overall QOL domain either. When metric data turned out to be normally distributed at baseline, mean and standard deviations of variables were calculated. Next, data imputations for missing scale values were carried out consecutively in three steps. Firstly, overall mean scores at baseline substituted missing baseline scale scores. Secondly, missing scores between two valid scores measured in time were individually interpolated. Thirdly, remaining missing values were imputed by Last Value Carried Forward procedure. Analysis on the resulting complete data was done by repeated measures $A N(C) O V A$ using 'time' as four categories, within-patients factor and both the experimental factor (control/intervention) as well as type of patient (child/adult) as dichotomous between factors. First and second order interactions between the covariate (baseline scale scores) and both factors were also tested. With regard to the clinical symptoms on at least four out of the seven-week days the scores had to be non-missing to obtain a valid total (morning or evening) symptom score for one week. In addition, at least three out of four weekly scores had to be non-missing to obtain a valid total (morning or evening) symptom score for one month. If these steps resulted in missing total monthly scores for individual patients, the same data imputation rules as for the AQLQ and PAQLQ were applied. Overall, AN(C)OVA tests were performed for testing 


\section{Chapter 4}

group differences in time with baseline scores as covariates. Missing data in medical consumption were imputed in the same way as described for the AQLQ and PAQLQ. One-month sum scores for medical consumption (medication excluded) were interpolated over one year by multiplying each month's score by 3 . The Mann-Whitney test was used on these interpolated one-year sum scores of the medical consumption. The Mann-Whitney test was used on one-year sum totals.

To explore the bivariate correlations between lung function values, morning and evening symptoms, scales of the AQLQ and PAQLQ, and beta-2 agonists and corticosteroids, Spearman's rank-order correlations were calculated. Partial rho rank correlations were calculated controlled for asthma severity using the GINA guidelines (10). Correlations between 0 and 0,2 very low, between 0,2 to 0,4 were considered low, between 0,4 to 0,6 moderate, between 0,6 to 0,8 high, and between 0,8 to 1 very high.

In all the analyses, a p-value of less than 0.05 was considered as statistically significant. All data analyses were done by SPSS-pc, version 12.0.1.

\section{Results}

\section{Data collection}

From patient records of the departments of Respiratory Medicine and Paediatrics 274 potentially eligible asthmatic outpatients were identified and approached by letter. Eighteen patients were not eligible because of the absence of a house phone connection $(7 \%)$, and 147 patients refused to participate in the study $(54 \%)$. The most frequently registered reasons for not participating were: 'having no time' (43\%), 'being uninterested' (19\%), 'not experiencing asthma symptoms' (12\%), and 'finding participation too confronting' (9\%). See the flowchart in figure 1.

The adults in the group who refused to participate were on average somewhat younger (41.30 years) and the children older (11.77 years) compared to the participants. With regard to gender there were on average more females in the children group (42\%) and fewer females in the adult group (50\%) compared to the participants. In the end, between January 2003 and January 2004, 109 patients (53 adults and 56 children) were included in the study. The intervention and control group appeared to be quite similar at baseline.

The mean age of the adults was 46 in both the control group and in the intervention group. The mean age of the children was 11 years in both groups. Of the adults $33 \%$ were male in the control group and $42 \%$ were male in the intervention group. In the children's control group, $56 \%$ of the patients were male, while $72 \%$ were male in the intervention group. 
Figure 1. Flowchart of study progress

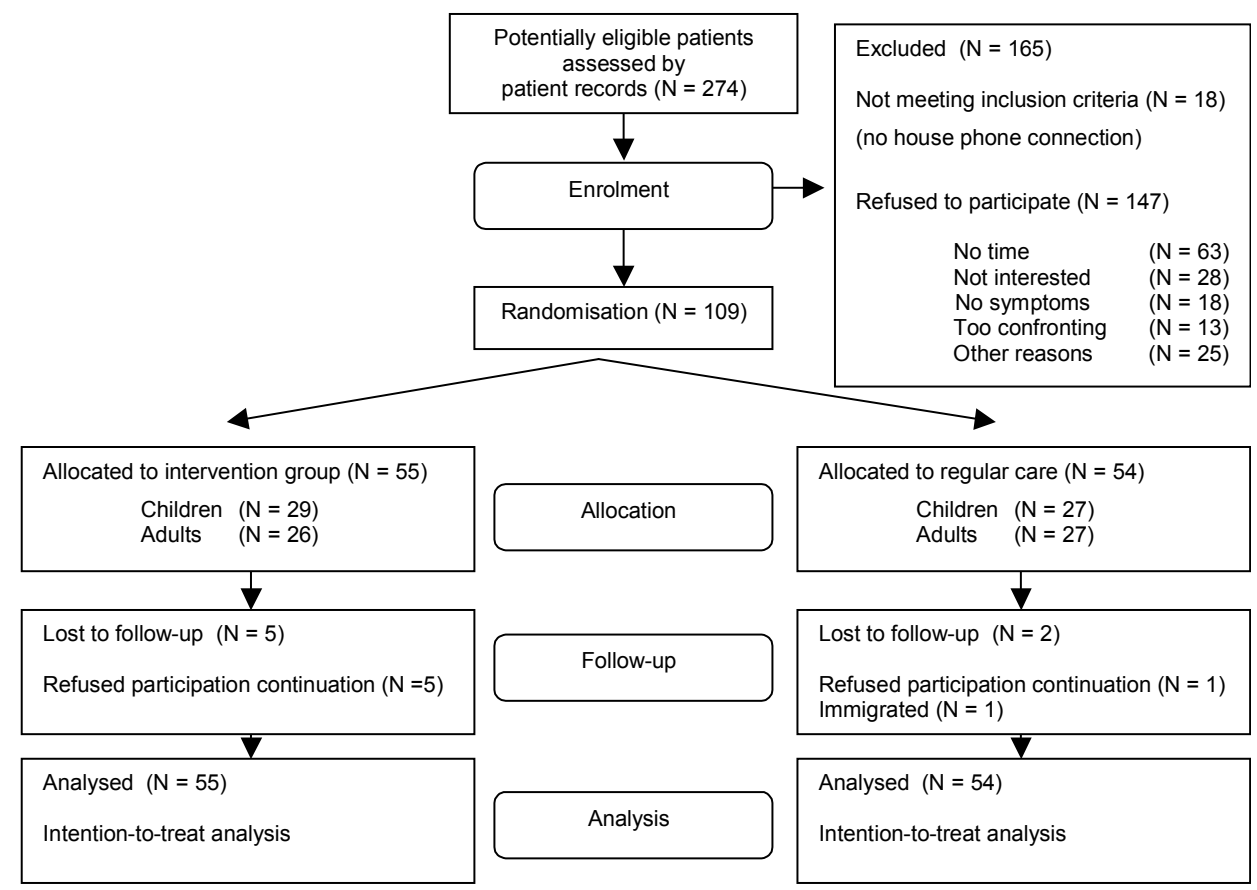

Among the adults, marital status and paid employment were somewhat higher in the intervention group. Lung function values show that the complete study population had reversible asthma. Of the 109 participants, seven patients (5 intervention group; 2 control group) were lost to follow-up. Six patients refused to continue the participation, and one patient migrated. Of the total group, an average of 1.5 tests was performed daily. The patient characteristics of the study population are presented in table 1. 
Chapter 4

Table 1. Baseline characteristics of the patients by age and group

\begin{tabular}{|c|c|c|c|c|c|c|c|c|c|c|c|c|}
\hline \multirow{2}{*}{$\begin{array}{l}\mathrm{N}=109 \\
\text { mean }(\mathrm{sd})\end{array}$} & \multicolumn{4}{|c|}{ Total } & \multicolumn{4}{|c|}{ Adults (18 years and older) } & \multicolumn{4}{|c|}{ Children (7-18 years) } \\
\hline & \multicolumn{2}{|c|}{$\begin{array}{l}\text { Control } \\
N=54\end{array}$} & \multicolumn{2}{|c|}{$\begin{array}{l}\text { Intervention } \\
\qquad \mathrm{N}=55\end{array}$} & \multicolumn{2}{|c|}{$\begin{array}{l}\text { Control } \\
\mathrm{N}=27\end{array}$} & \multicolumn{2}{|c|}{$\begin{array}{l}\text { Intervention } \\
\qquad N=26\end{array}$} & \multicolumn{2}{|c|}{$\begin{array}{l}\text { Control } \\
\mathrm{N}=27\end{array}$} & \multicolumn{2}{|c|}{$\begin{array}{c}\text { Intervention } \\
\qquad N=29\end{array}$} \\
\hline age & 28.38 & $(21.0)$ & 27.16 & (19.3) & 45.90 & $(15.9)$ & 45.65 & $(11.3)$ & 10.85 & $(2.3)$ & 10.57 & $(2.1)$ \\
\hline lung function & & & & & & & & & & & & \\
\hline FVC \% pred & 100.6 & $(16.5)$ & 100.2 & $(12.3)$ & 102.5 & (15.3) & 104.2 & $(14.7)$ & 98.7 & $(17.7)$ & 96.5 & $(13.7)$ \\
\hline FEV1 \% pred & 96.0 & (16.4) & 94.9 & $(17.7)$ & 92.4 & (19.9) & 92.6 & $(21.4)$ & 99.4 & $(11.3)$ & 96.5 & $(8.4)$ \\
\hline FEV1 \% VC ref & 79.6 & $(11.0)$ & 77.9 & (11.8) & 75.2 & $(10.4)$ & 73.0 & $(12.8)$ & 84.1 & $(9.8)$ & 82.4 & $(8.9)$ \\
\hline PEF \% pred & 95.5 & (20.1) & 99.4 & $(32.5)$ & 99.3 & $(23.8)$ & 108.4 & $(42.9)$ & 91.7 & $(14.9)$ & 91.3 & $(16.0)$ \\
\hline $\begin{array}{l}\text { GINA* } \\
\text { gender }\end{array}$ & 2.41 & $(0.8)$ & 2.62 & $(0.7)$ & 2.74 & $(0.7)$ & 2.96 & $(0.5)$ & 2.07 & $(0.7)$ & 2.31 & $(0.8)$ \\
\hline male & 44.4 & $\%$ & 58.2 & $\%$ & 33.3 & $\%$ & 42.3 & $\%$ & 55.6 & $\%$ & 72.4 & $\%$ \\
\hline $\begin{array}{l}\text { female } \\
\text { civil status }\end{array}$ & 55.6 & $\%$ & 41.8 & $\%$ & 66.7 & $\%$ & 57.7 & $\%$ & 44.4 & $\%$ & 27.6 & $\%$ \\
\hline single & 11.1 & $\%$ & 3.6 & $\%$ & 22.2 & $\%$ & 7.7 & $\%$ & 0 & $\%$ & 0 & $\%$ \\
\hline with parents & 53.7 & $\%$ & 52.7 & $\%$ & 7.4 & $\%$ & 0 & $\%$ & 100 & $\%$ & 100 & $\%$ \\
\hline $\begin{array}{l}\text { married/living } \\
\text { together }\end{array}$ & 35.2 & $\%$ & 43.7 & $\%$ & 77.4 & $\%$ & 92.3 & $\%$ & 0 & $\%$ & 0 & $\%$ \\
\hline main daily activity & & & & & & & & & & & & \\
\hline paid employment & 22.2 & $\%$ & 30.9 & $\%$ & 44.4 & $\%$ & 65.4 & $\%$ & 0 & $\%$ & 0 & $\%$ \\
\hline sick/disabled & 13.0 & $\%$ & 5.4 & $\%$ & 25.9 & $\%$ & 11.5 & $\%$ & 0 & $\%$ & 0 & $\%$ \\
\hline school/college & 51.9 & $\%$ & 52.7 & $\%$ & 3.7 & $\%$ & 0.0 & $\%$ & 100 & $\%$ & 100 & $\%$ \\
\hline housekeeping & 5.6 & $\%$ & 3.6 & $\%$ & 11.1 & $\%$ & 7.7 & $\%$ & 0 & $\%$ & 0 & $\%$ \\
\hline other & 7.4 & $\%$ & 3.6 & $\%$ & 14.8 & $\%$ & 15.4 & $\%$ & 0 & $\%$ & 0 & $\%$ \\
\hline
\end{tabular}

FVC \% pred = forced vital capacity expressed as a percentage of predicted; FEV1 \% pred = forced expiratory volume in 1 second expressed as a percentage of predicted; FEV1 \% VC ref = forced expiratory volume expressed as a percentage of predicted / vital capacity; PEF \% pred = peak expiratory flow expressed as a percentage of predicted.

* GINA classification based on prescribed medication; 1 = intermittent asthma, 2 = persistently mild asthma, 3 = persistently moderate asthma, 4 = persistently severe asthma.

\section{Asthma-specific quality of life scores}

Table 2 provides means and standard deviations of 3 domains from the PAQLQ and the $A Q L Q$ during the four measurements for both groups.

Overall, the scores in the (P)AQLQ are relatively high which implies that the selected sample shows mild to moderate, but no severe asthma. Table 2 shows that although patients in both groups did improve in time in all domains, no statistically significant differences were found between the groups. 
The effectiveness of nurse-led telemonitoring of asthma

Table 2. One-year follow-up of asthma-specific quality of life and self-reported symptoms

\begin{tabular}{|c|c|c|c|c|c|c|c|c|c|c|c|c|}
\hline \multirow{3}{*}{$\begin{array}{l}(\mathrm{P}) \mathrm{AQLQ}^{1} \\
\text { Control N=54 } \\
\text { Intervention } \mathrm{N}=55 \\
\text { month } 1\end{array}$} & \multicolumn{4}{|c|}{ Symptoms } & \multicolumn{4}{|c|}{ Activity limitation } & \multicolumn{4}{|c|}{ Emotional function } \\
\hline & \multicolumn{2}{|c|}{ Control } & \multicolumn{2}{|c|}{ Intervention } & \multicolumn{2}{|c|}{ Control } & \multicolumn{2}{|c|}{ Intervention } & \multicolumn{2}{|c|}{ Control } & \multicolumn{2}{|c|}{ Intervention } \\
\hline & 5.42 & $(1.12)$ & 5.44 & $(1.24)$ & 5.01 & $(1.33)$ & 5.08 & $(1.10)$ & 6.21 & $(0.85)$ & 6.10 & $(1.01)$ \\
\hline month 4 & 5.45 & $(1.13)$ & 5.76 & $(1.01)$ & 5.05 & $(1.27)$ & 5.21 & $(1.16)$ & 6.24 & $(0.90)$ & 6.29 & $(0.78)$ \\
\hline month 8 & 5.49 & $(1.07)$ & 5.68 & $(1.23)$ & 5.17 & $(1.13)$ & 5.16 & $(1.18)$ & 6.29 & $(0.80)$ & 6.19 & $(1.00)$ \\
\hline month 12 & 5.48 & $(1.18)$ & 5.73 & $(1.09)$ & 5.21 & $(1.32)$ & 5.13 & $(1.17)$ & 6.28 & $(0.81)$ & 6.39 & $(0.76)$ \\
\hline$p$-values group ${ }^{2}$ & \multicolumn{4}{|c|}{$<.001$} & \multicolumn{4}{|c|}{$<.001$} & \multicolumn{4}{|c|}{$<.001$} \\
\hline time $x$ group $^{3}$ & \multicolumn{4}{|c|}{.386} & \multicolumn{4}{|c|}{.640} & \multicolumn{4}{|c|}{.245} \\
\hline \multirow[t]{2}{*}{$\begin{array}{l}\text { morning } \\
\text { symptoms }\end{array}$} & \multicolumn{4}{|c|}{ Coughing } & \multicolumn{4}{|c|}{ Production of sputum } & \multicolumn{4}{|c|}{$\begin{array}{l}\text { Shortness of breath } \\
\text { / wheezing }\end{array}$} \\
\hline & \multicolumn{2}{|c|}{ Control } & \multicolumn{2}{|c|}{ Intervention } & \multicolumn{2}{|c|}{ Control } & \multicolumn{2}{|c|}{ Intervention } & \multicolumn{2}{|c|}{ Control } & \multicolumn{2}{|c|}{ Intervention } \\
\hline month 1 & .48 & $(.52)$ & .34 & $(.32)$ & .24 & $(.30)$ & .22 & $(.37)$ & & $(.56)$ & .34 & $(.55)$ \\
\hline month 4 & .55 & $(.57)$ & .26 & $(.36)$ & .32 & (.43) & .17 & $(.31)$ & .47 & (.65) & .25 & $(.40)$ \\
\hline month 8 & .45 & $(.50)$ & .26 & $(.43)$ & .30 & $(.44)$ & .20 & $(.36)$ & .48 & (.61) & .33 & $(.50)$ \\
\hline month 12 & .41 & (.49) & .23 & (.38) & .27 & $(.44)$ & .18 & $(.31)$ & .40 & $(.48)$ & .27 & $(.45)$ \\
\hline$p$-values group ${ }^{2}$ & \multicolumn{4}{|c|}{.001} & \multicolumn{4}{|c|}{.067} & \multicolumn{4}{|c|}{.357} \\
\hline time $x$ group $^{3}$ & \multicolumn{4}{|c|}{.245} & \multicolumn{4}{|c|}{.191} & & & 31 & \\
\hline $\begin{array}{l}\text { evening } \\
\text { symptoms }\end{array}$ & & Coug & hing & & & oduction & of sput & & & $\begin{array}{r}\text { ortnes } \\
\text { / whe }\end{array}$ & $\begin{array}{l}\text { of breatl } \\
\text { ezing }\end{array}$ & \\
\hline & Cor & trol & Interv & ention & Cor & trol & Interv & ention & Con & trol & Interve & ntion \\
\hline month 1 & .61 & $(.62)$ & .50 & $(.52)$ & .38 & $(.44)$ & .32 & $(.47)$ & .61 & $(.65)$ & .44 & $(.60)$ \\
\hline month 4 & .63 & $(.71)$ & .40 & (.53) & .43 & $(.60)$ & .25 & $(.42)$ & .64 & (.83) & .32 & $(.43)$ \\
\hline month 8 & .49 & $(.52)$ & .40 & $(.58)$ & .33 & $(.44)$ & .30 & $(.52)$ & .62 & (.73) & .42 & (.61) \\
\hline month 12 & .48 & $(.54)$ & .41 & (.61) & .34 & $(.46)$ & .25 & $(.40)$ & .57 & (.65) & .35 & $(.55)$ \\
\hline$p$-values group ${ }^{2}$ & & .02 & & & & .1 & & & & & & \\
\hline time $x$ group ${ }^{3}$ & & .15 & & & & & & & & & & \\
\hline
\end{tabular}

\section{Symptom scores}

The symptoms coughing, production of sputum, and shortness of breath / wheezing are separately presented as morning and evening symptoms in table 2 . On average, the frequency of symptoms was low. Coughing and shortness of breath / wheezing were registered somewhat more frequently than the production of sputum. The mean frequencies of the evening scores were somewhat higher than the morning scores on all measurements. In time, the morning as well as the evening symptom 'coughing' improved in both groups. However, no statistically significant differences in improvement in any of the symptoms were observed between the groups. 


\section{Chapter 4}

\section{Medical consumption}

The interpolated data on medical consumption specified for type of care provider and intervention group per hundred are presented in figure 2. The data show that hospitalisation did not occur during the 12 months, and four patients in the control group visited the emergency room during the study year. The total summated medical consumption as reported in the diaries seemed higher in the control group, except for the consultations of the asthma nurse. No significant differences were observed among the types of medical consumption between 12 months and baseline scores. No statistical differences were found between the groups in the total scores (Mann-Whitney p-value > 0.05 ; figure 2).

Figure 2. Cluster bar of medical consumption specified in both groups for type of care provider and medication per hundred patients cumulated over one year

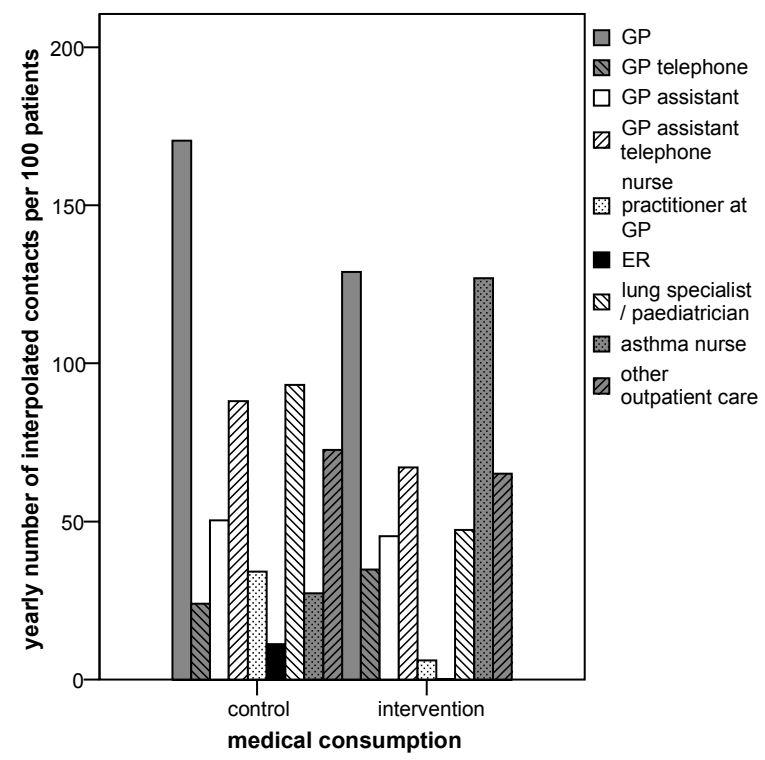

In the control group ( $\mathrm{N}=54$ ) cumulated over one year, 22 patients consumed primary care, 19 patients secondary care, and 53 patients were using medication. In the intervention group $(\mathrm{N}=55)$ cumulated over one year, 28 patients consumed primary care, 19 patients secondary care, and 52 patients used medication. The stacked bars in figure 3 represent the relative amount of patients who consumed medical care or used medication. 
Figure 3. Stacked bars of proportions of patients with medical consumption specified in both groups for primary care, secondary care and medication cumulated over one year
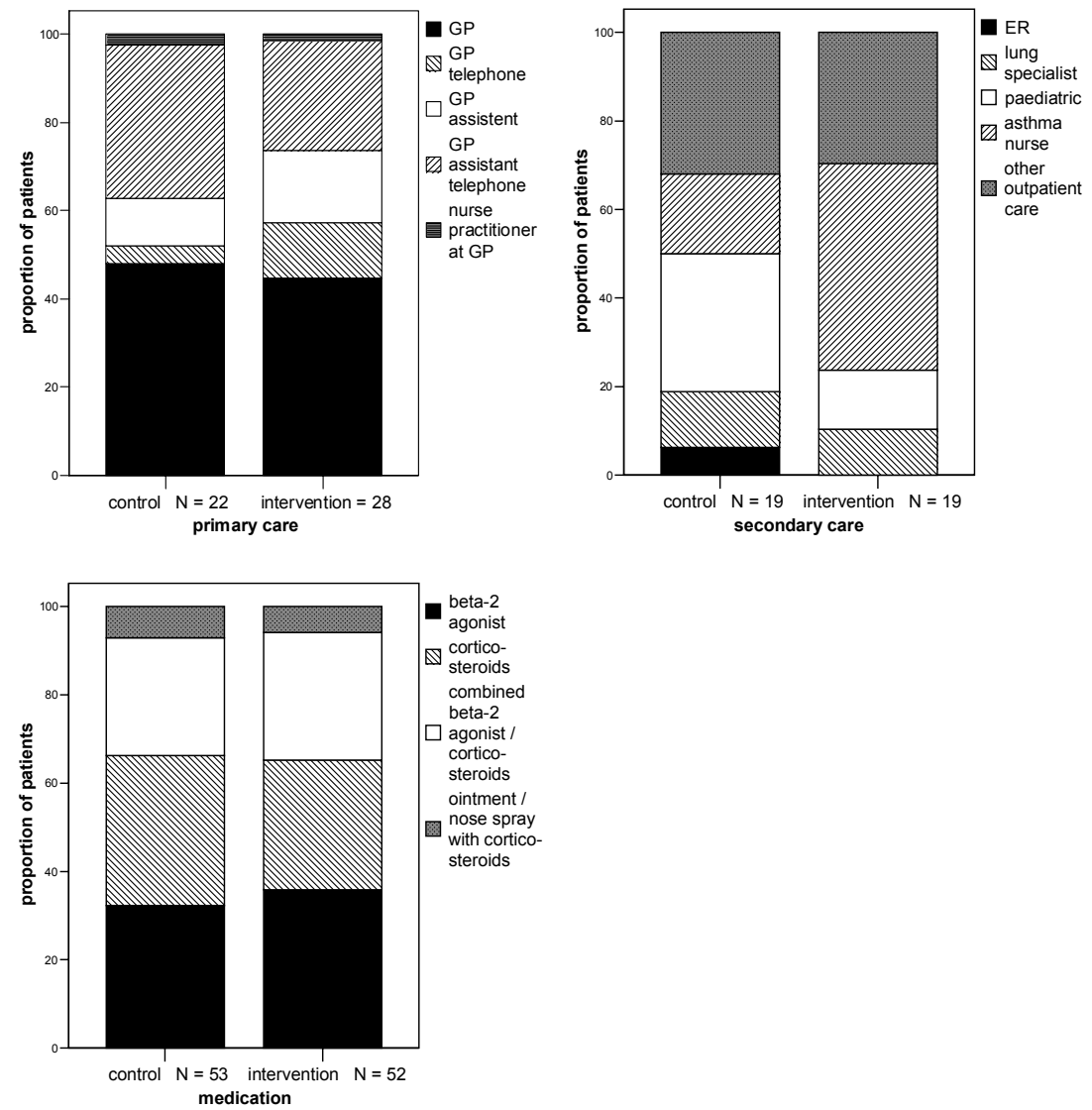

With regard to primary health care, the stacked bars in figure 3 show that the proportions of telephone contacts with the general practitioner and consultations with the general practitioners' assistant (including repeat prescriptions) were higher in the intervention group. Telephone contacts with the general practitioners assistant occurred relatively more often in the control group. In secondary care no emergency visits occurred in the intervention group, and paediatric consultations occurred relatively less often in this group. In the intervention group the proportion of contacts with the outpatient asthma nurse linked to the telemonitoring programme, was higher. 


\section{Chapter 4}

The self-reported use proportion of beta-2 agonists, corticosteroids, combined medication (beta-2 agonist and corticosteroids), and ointment and/or nose spray medication consumption is similar in both groups. No statistically significant differences were found between the groups in the annual amount of medication used (Mann-Whitney p-value $>0.05$ ).

\section{Relationship between outcome parameters}

Baseline rank correlations between lung function values (FEV1 and PEF), clinical symptoms, (P)AQLQ domains (Symptoms, Activity limitation, and Emotional Function) and medication (beta-2 agonists and corticosteroids) are presented in table 3.

The rank correlations of lung function values (PEF and FEV1), of morning symptoms and evening symptoms, and of (P)AQLQ and medication were on average moderate to high. Morning asthma symptoms and evening asthma symptoms also correlated highly with each other. All these correlations were statistically significant.

With regard to correlations between the outcome parameters, no significant correlations were found between asthma symptoms and the other outcome parameters.

Low correlations were found between the domain Activity Limitation of the (P)AQLQ and the lung function values (FEV1 and PEF). Furthermore, low and statistically significant correlations were present between all the domains of the (P)AQLQ and beta-2 agonists, while there were very low, non-significant correlations between the (P)AQLQ and corticosteroids.

The lung function value PEF \% predicted correlated very low and statistically significant with the self-reported use of corticosteroids, and correlated not statistically significant with the self-reported use of beta-2 agonists. The original rank correlations remained the same with similar $p$-values in the partial analyses controlled for asthma severity (GINA guidelines). 


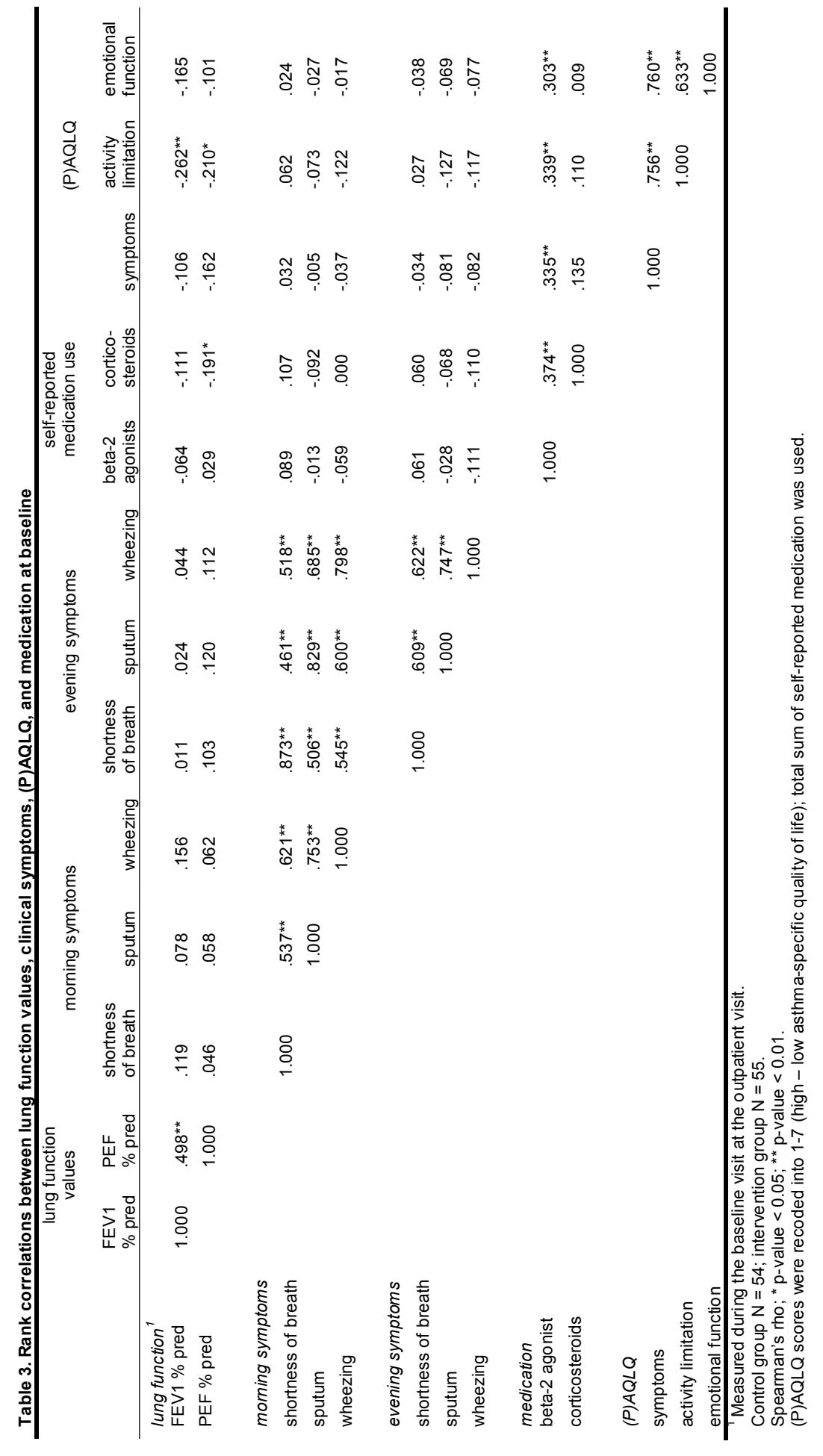




\section{Chapter 4}

\section{Discussion}

This study evaluates the effects and relation between the outcome parameters of a nurse-led telemonitoring intervention in asthmatics in terms of clinical symptoms, asthma-specific quality of life, and medical consumption.

The study population generally had mild to moderate asthma. The results of this study show improvement in time in all three domains of asthma-specific quality of life in both groups. However, against the expectation no statistically significant differences in improvement in asthma-specific quality of life were observed between the patients who received the nurse-led telemonitoring programme and the patients who received regular care. Also, no statistically significant differences in change were observed between the groups in self-reported asthma symptoms. Not surprisingly, the intervention group consulted the asthma nurse who was the main care provider, more often than the regular care group. Further, emergency room visits only occurred in the control group. However, these differences in medical consumption were not significant between the groups. Also, with regard to the amount of self-reported medication the groups did not differ significantly. The overall test of AN(C)OVA with age group as covariate shows that differences in effects between the groups are similar in both age groups. This implies that there is a parallel development of no significant differences in improvement between the intervention group and the regular care group in the adults and the children.

The correlations between PEF and FEV1, between symptom scores, between corticosteroids and beta-2 agonists, and between the domains of asthma-specific quality of life show on average moderate to high correlations in the expected direction. All these internal correlations were statistically significant. The lung function value PEF has a very low correlation with the self-reported use of corticosteroids and the PEF and FEV1 has a low correlation with the domain Activity Limitation of the (P)AQLQ, as expected. Very low, non-significant correlations are present between the symptoms and the other outcome parameters, though we expected low correlations with the dimensions of asthma-specific quality of life. With regard to medication, very low and non-significant correlations were found between the domains of asthma-specific quality of life and the self-reported use of corticosteroids, but remarkably low and statistically significant correlations were found between all three domains of asthma-specific quality of life and self-reported use of beta- 2 agonists. This suggests that the use of beta- 2 agonists relates stronger to asthma-specific quality of life than to self-reported symptoms.

There are some limitations in this study, such as the participation rate. The participation $(40 \%)$ is not overwhelming, even though the patient's burden is restricted to a minimum. Nevertheless, there are no indications that the study population is not a representative 
sample of asthmatic outpatients with mild to moderate asthma. 'Having no time' is the main reason for not participating in this programme. This indicates that it is very important that future telemonitoring programmes are as little time-consuming as possible. We speculate that in a more severe asthma population the improvement of clinical symptoms, the asthma-specific quality of life, and the decrease of medical consumption may be more impressive and therefore more room for improvement. Besides this, the participation rate will probably be higher, as patients and parents are more confronted with asthma symptoms and their daily functioning is more limited. With regard to the data, missing scale values are imputed. Repeating the analyses without data imputation yields the same results. This study combines the asthma-specific quality of life questionnaires (AQLQ and PAQLQ). Although there are no examples of this in the literature, as the three domains contain equivalent items in both questionnaires, we believe that this combination is valid.

In the review by Ouwens et al. (23) important requirements for a successful implementation for management programmes of patients with chronic illnesses are described. These requirements include a shared mission on integrated care between the professionals involved, and motivated patients for self-management. Other contiguous studies emphasise the continual improvement of the processes (24), efficiently organised care processes, optimal teamwork, and collaboration (25). The results of this study can therefore serve as a handle for implementation strategies for self-management programmes in asthma. In this study, the main care provider in the team was an asthma nurse. A lung specialist and a paediatrician were available for consultations, if necessary. The general practitioners were informed about the patients' participation, but there was no direct form of cooperation. Furthermore, there were no protocol-led engagements between the members of the telemonitoring programme and the pharmacists. The fact that not all care providers participated in the telemonitoring programme, and that the participation rate was relatively low, may partially explain why no statistical differences in medical consumption were found between the groups. Selfmanagement programmes themselves may lead to fewer symptoms and improved quality of life, as reported in many other studies (17-20). Nevertheless, as long as interventions are initiated separately instead of integrated into the total process of care, the intervention may only partially contribute to the improvement of the total care process. This highlights the importance of integrated care in self-management programmes, such as telemonitoring programmes.

A remarkable finding of this study is the correlation between beta- 2 agonists and asthma-specific quality of life, and no correlation between corticosteroids and asthma- 


\section{Chapter 4}

specific quality of life. Beta-2 agonists are more commonly known as 'relievers'; the drugs relax and open the airways in the lungs in the short-term. The domains of the asthma-specific quality of life ((P)AQLQ) express a person's daily functioning. This could suggest that the impact of asthma on functioning is of more significance in administrating beta-2 agonists than the current perception of clinical asthma symptoms. This is in line with the application of beta- 2 agonists, since they can be used with great advantages for the patients when they experience asthma symptoms, or in advance of exercise. A possible explanation for the relation between the beta-2 agonist and the asthma-quality of life domains could therefore be that the use of beta- 2 agonists is highly dependent on the patient's planned activities. This implies that asthma patients are strongly focussed on the prevention and relief of asthma symptoms, so that they can continue to function as normally as possible. Not a symptom-free state, but the maximisation of daily functioning should be the goal of asthma telemonitoring programmes. This point of view on asthma behaviour is a very interesting starting point for care providers in the asthma field. This is in harmony with the patient-centred point of departure which is one of the health care aims of the Disease Management Association of America (26).

Overall, the telemonitoring programme evaluated in this study does not significantly decrease asthma symptoms or medical consumption, or improve asthma-specific quality of life. This is consistent with the results of a study by Buist et al. (27] in which no significant differences were found among older adults with moderate to severe asthma between peak flow rate and symptom monitoring, or between twice-daily and as-needed peak flow monitoring in health care utilisation, asthma specific quality of life, and lung function. However, one should bear in mind that among other relevant outcome parameters which are not described in the studies, such as patient satisfaction, effects may be present.

In conclusion, investigating the relation between outcome parameters indicates that patients' perception of asthma-specific quality of life (domains: Symptoms, Activity limitation, and Emotional Function) should be a key element in asthma telemonitoring programmes. The results of this study also emphasise that a telemonitoring programme on its own is not a guarantee of success. Telemonitoring programmes have to dovetail with the needs, and the behaviour of the patients concerned. Only this way can disease management programmes maximise the effects. 


\section{References}

1. World Health Organization (WHO). Available from: http://www.who.int/mediacentre /factsheets/fs307/en/index. $\mathrm{html}$

2. Garg VK, Bidani R, Rich EP, Hershey E, Hershey CO. Asthma patients' knowledge, perception, and adherence to the asthma guidelines. J Asthma. 2005; 42:633-8.

3. Cote J, Cartier A, Malo JL, Rouleau M, Boulet LP. Compliance with peak expiratory flow monitoring in home management of asthma. Chest. 1998; 113:968-72.

4. Verschelden P, Cartier A, L'Archeveque J, Trudeau C, Malo JL. Compliance with and accuracy of daily selfassessment of peak expiratory flows (PEF) in asthmatic subjects over a three month period. Eur Respir $\mathrm{J}$. 1996; 9:880-5.

5. Horrocks S, Anderson E, Salisbury C. Systematic review of whether nurse practitioners working in primary care can provide equivalent care to doctors. BMJ. 2002; 324:819-23.

6. Goodfellow LT, Rau JL. Devices and adjuncts to self-management for asthma sufferers. Business briefing: long-term health care strategies. Business briefing: long-term health care strategies; 2003. Available from: http://www.touchbriefings.com/pdf/14//th031_r_goodfellow.PDF

7. Ignacio-Garcia JM, Gonzales-Santos P. Asthma self-management education program by home monitoring of peak expiratory flow. Am J Respir Care Med. 1995; 151:353-9.

8. Lahdensuo A, Haahtela T, Herrala J, Kava T, Kiviranta K, et al. Randomised comparison of guided self management and traditional treatment of asthma over one year. BMJ. 1996; 312:748-52.

9. Willems DC, Joore MA, Hendriks JJ, Wouters EF, Severens JL. Cost-effectiveness of self-management in asthma: A systematic review of peak flow monitoring interventions. Int J Technol Assess Health Care. 2006; 22:436-42.

10. Masoli M, Fabian D, Holt S, Beasley R. Global Initiative For Asthma (GINA): global burden of asthma; 2004. Available from: http://www.ginasthma.com/Reportltem.asp?!1=2\&/2=2\&intld=94

11. Juniper EF, Guyatt GH, Ferrie PJ, Griffith LE. Measuring quality of life in asthma. Am Rev Respir Dis. 1993; 147:832-8.

12. Juniper EF, Guyatt GH, Feeny DH, Ferrie PJ, Griffith LE, et al. Measuring quality of life in children with asthma. Qual Life Res. 1996; 5:35-46.

13. Juniper EF, Guyatt GH, Feeny DH, Ferrie PJ, Griffith LE, et al. Measuring quality of life in the parents of children with asthma. Qual Life Res. 1996; 5:27-34.

14. Geijer RMM, Van Hensbergen W, Bottema BJAM, van Schayck CP, Sachs APE, et al. NHG-Standaard Astma bij volwassenen: behandeling (in Dutch). Huisarts Wet. 2001; 44:153-164.

15. Dirksen WJ, Geijer RMM, De Haan M, De Koning G, Flikweert S, et al. NHG-Standaard Astma bij kinderen (in Dutch). Huisarts Wet. 1998; 41:130-43.

16. Hendriks JJE, Wesseling GJ, Van Duurling RAH, Wouters EFM. Werkprotocol: Patient research in selfmanagement of asthma (in Dutch). University Hospital Maastricht; 2001.

17. Gibson PG, Powell H, Coughlan J, Wilson AJ, Abramson M, et al. Self-management education and regular practitioner review for adults with asthma (Cochrane Review). Cochrane Library, Issue 3, DOI:10.1002/14651858.CD001117; 2002.

18. Gibson PG, Powell H, Coughlan J, Wilson AJ, Hensley MJ, et al. Limited (information only) patient education programs for adults with asthma (Cochrane Review). Cochrane Library, Issue 3, DOI:10.1002/14651858.CD001005; 2002.

19. Gibson PG, Ram FSF, Powell H. Asthma education. Respir Med. 2003; 97:1036-1044.

20. Guevara JP, Wolf FM, Grum CM, Clark NM, Cates CJ. Effects of educational interventions for selfmanagement of asthma in children and adolescents: systematic review and meta-analysis. Br Med J. 2003; 326:1308-9. 


\section{Chapter 4}

21. Juniper EF, Kline PA, Vanzieleghem MA, Ramsdale EH, O'Byrne PM, et al. Effect of long-term treatment with an inhaled corticosteroid (budesonide) on airway hyperresponsiveness and clinical asthma in nonsteroiddependent asthmatics. Am Rev Respir Dis. 1990; 142:832-6.

22. Apter AJ, ZuWallack RL, Clive J. Common measures of asthma severity lack association for describing its clinical course. J Allergy Clin Immunol. 1994; 94:732-7.

23. Ouwens M, Wollersheim H, Hermens R, Hulscher M, Grol R. Integrated care programmes for chronically ill patients: a review of systematic reviews. Int J Qua Health Care. 2005; 17:141-6.

24. Shortell SM, Bennett CL, Byck GR. Assessing the impact of continuous quality improvement on clinical practice: what it will take to accelerate progress. Milbank Q. 1998; 76:593-624.

25. Grol R. Between evidence-based practice and total quality management: the implementation of cost-effective care. Int J Qual in Health Care. 2000; 12:297-304.

26. Disease management Association of America. Available from: htpp://www.dmaa.org

27. Buist AS, Vollmer WM, Wilson SR, Frazier EA, Hayward AD. A randomized clinical trial of peak flow versus symptom monitoring in older adults with asthma. Am J Respir Crit Care Med. 2006; 174:1077-1087. 
Chapter 5

Using EQ-5D in children with a chronic condition Submitted

DCM Willems MA Joore

FHM Nieman

JL Severens

EFM Wouters

JJE Hendriks 


\section{Chapter 5}

\section{Abstract}

Objective: This study is the first to explore the use of the EQ-5D child version in children with chronic conditions.

Study design: A convenience sample was selected from hospital outpatient records and school records. The sample included children aged 7-18 years with the following chronic conditions: asthma, rheumatic disorders, diabetes, and speech/language/hearing disorders. The feasibility, construct validity and discriminant power were compared with a generic quality of life questionnaire for children (TACQOL) and the 2-week test-retest reliability was assessed.

Results: In the end, 182 of these children or their parents completed the first questionnaire and 161 children/parents completed both questionnaires. The feasibility of the EQ-5D was good. The low to moderate correlations suggest that the EQ-5D and TACQOL measure different concepts. The discriminant power was low overall. The EQ$5 \mathrm{D}$ items discriminated the most between children with a rheumatic disorder and children with the other conditions. In the subset of children who experienced no health change between the test and the retest the reliability was moderate to high.

Conclusions: It appears that the EQ-5D is not directly suitable for children. The EQ-5D seems the most suitable for children with a chronic physical condition and appears to be reliable for children with a stable health status. 


\section{Introduction}

Increasingly, the health consequences of interventions are assessed in terms of the impact on health-related quality of life.

The preferences individuals or society have for any particular set of health outcomes is referred to as utility (1). The change in utility combined with life expectancy (QualityAdjusted Life Year) is commonly used to express the main economic benefit of a health intervention. To measure and score utilities, multi-attribute utility instruments have been developed. These days there is a growing demand for child health assessment tools (2). The EQ-5D is extensively used for adults and this questionnaire has been proven to be reliable and valid (3). Up to now the validity of the EQ-5D in a juvenile population has only been investigated in children treated for imperforate anus (4). That study supported the validity of the EQ-5D with regard to these children.

To our knowledge, no study has explored the use of the EQ-5D child version in children with chronic conditions. A chronic condition is an illness or impairment which has persistent or recurring health consequences which affect the emotional and physical wellbeing. If it is not possible to cure a condition but management of the condition is the main goal of the treatment, it must be determined how far the treatment and the condition compromise the child's quality of life (5). Chronic diseases are the primary cause of mortality in the world, representing $60 \%$ of all deaths. In 200535 million people suffered from chronic disease, half of whom were under 70 years old and half were women (6). Of the Dutch population $10 \%$ to $20 \%$ is estimated to suffer from a chronic condition (7). The extent to which a condition affects people's lives is different for each person. Also, the way children with a chronic condition cope and adjust is dependent on the child's developmental stage. As the burden experienced by a condition and the course of each chronic condition is so diverse, multiple conditions have been included in this study.

The objective of this explorative study was to examine the use of the EQ-5D in children with chronic conditions. The chronic conditions included asthma, diabetes, rheumatic disorders, and speech/language/hearing disorders. For this purpose the feasibility, construct validity, discriminatory power and test-retest reliability were investigated.

\section{Patients and methods}

\section{Study population}

A convenience sample of the population was selected by means of hospital outpatient records and school records. Those included were children aged 7-18 years with one of 


\section{Chapter 5}

the following four conditions: asthma, rheumatic disorders, diabetes, or speech/language /hearing disorders. Children with asthma (asthma severity stage I - III), a rheumatic disorder or diabetes were recruited from the outpatient clinics of the University Hospital Maastricht (Maastricht; the Netherlands) and the Hospital Gooi-Noord (Blaricum; the Netherlands). Children with a speech/language/hearing disorder were recruited from the outpatient clinic of the University Hospital Maastricht or were pupils of the Mgr. Hanssenschool (Hoensbroek; the Netherlands). This is a special primary school for children with a speech/language disorder in combination with another disorder, such as hearing impairment or mental retardation.

\section{Instruments}

The EQ-5D consists of a descriptive system (EQ-5D) and a visual analogue scale (VAS) on which patients rate their own health between 0 and 100 (3). The five questions in the EQ-5D classify persons into one of 243 health states. The commonly used scoring function for the EQ-5D is based on a British study (EQ-5D UK) with preferences derived by the time trade-off-method, in a representative sample of the adult UK population (3). The possible range of utility scores (global score) is from -0.59 (lowest) to 1.00 (highest). This study used the EQ-5D child version as previously used by Stolk et al. which entails a child form and a parent form (4). These forms are based on the 5 items of the (adult) $E Q-5 D$ and the VAS. The questions are geared toward children, consequently there are some slight alterations in the phrasing. However, the interpretation of the scores and the calculation of the utility scores are similar to the standard EQ-5D (adult version). The TNO-AZL Questionnaires for Children's Health-Related Quality of Life (TACQOL) was developed to measure generic quality of life in children aged between 5 and 15 with chronic diseases and contains seven scales, each consisting of eight items $(8,9,10)$. There is a parent form for children aged 6-15 years and a child form for children aged 815 years. Except for the phrasing of the items, their design and scale structure are identical and the two forms can be used as supplementary measures. The seven scales measure: limitations in physical functioning, motor functioning, autonomy, cognition, social, and the occurrence of both positive and negative emotions. The TACQOL scales were calculated by the use of a SPSS programme as described in the TACQOL manual (11).

\section{Procedure}

Children aged 12 years and older completed both the EQ-5D child form and the TACQOL child form by themselves (with or without help of their parents). For children 
younger than 12 years of age the parents or caregivers completed the parent form (with or without help from the children). All children and parents received an information letter and after obtaining informed consent the first questionnaires were sent by post. This first questionnaire concerned the EQ-5D child version and the TACQOL. After 2 weeks they received the second questionnaire, a second EQ-5D child version. Additional data that was requested concerned the child's age, the gender, the date of completion, qualitative comments, and the administration method (with or without help of the child or parent). A question regarding perceived changes in the health situation was added during the study.

\section{Statistic analyses}

The feasibility of the EQ-5D child version was indicated by describing the qualitative comments during the procedure and by counting the number of missing baseline scores on the EQ-5D, VAS, and TACQOL scales. With regard to the TACQOL this implied that if more than two of the eight scale item scores were missing the overall scale score was defined as missing. As the $E Q-5 D$ is a simple and short questionnaire, it was expected that the feasibility would be good. The construct validity was measured by calculating Spearman rank correlations (rho) between the EQ-5D (EQ-5D items, VAS and EQ-5D utility) and the TACQOL scales. The rank correlations were expected to be moderate to high $(0.40 \leq$ rho $\leq 0.80)$ between the corresponding concepts in the EQ-5D and the TACQOL. The discriminant power of the EQ-5D and TACQOL was explored by examining the univariate statistics of the scale scores between the conditions. As rheumatic disorders are characterised by stiffness or limitation of motion this group of children was expected to experience more problems with regard to mobility and selfcare. Apart from stiffness or limitation of motion, rheumatic disorders are often also accompanied by pain. Among asthmatic children exercise-induced asthma is common and can lead to shortness of breath. For these reasons it was expected that children with asthma or a rheumatic disorder would experience the most limitations with regard to their usual activities and experience more pain/discomfort. As communication disorders have the potential to isolate individuals from their social surroundings, feelings of anxiety/depression were expected to be the most present among children with a speech/language/hearing disorder. To investigate the reproducibility of the EQ-5D interclass correlation coefficients (ICCS) were used for measuring the test-retest reliability on two repeated measurements. Due to the large variability in the course of asthma and rheumatic disorders, the lowest ICCs were expected in this group. In addition, ICCs were calculated for the subset of children who stated that they had 


\section{Chapter 5}

experienced no health changes between the two questionnaires and for the subset of children who did experience health changes. Statistical analysis was performed using SPSS, version 12.0. A p-value of 0.05 was considered to be statistically significant.

\section{Results}

\section{Study population}

Of the 368 children who were invited to participate 194 (53\%) children were willing to participate in the study. In the end, 182 of these children or their parents completed the first questionnaire and 161 children or their parents completed both the first and second questionnaire. On average the second questionnaire was returned after 15 days.

Table 1 shows these figures in more detail. More children aged 7-12 years than children aged 12-18 years were included for each condition. This was especially the case with regard to children with a speech/language/hearing disorder, due to the fact that these children were mainly recruited from a special primary school. In at least half of the children the cause of the speech and/or language disorder was a hearing impairment. The mean age among the 99 participants aged 7-12 years who completed both questionnaires was 10 (7-12; SD 1.5) and 59\% were boys.

Among the 62 children aged 12-18 years who completed both questionnaires, the mean age was 15 (12-18; SD 1.8) and $48 \%$ were boys.

Table 1. Study population by condition and age group

\begin{tabular}{|c|c|c|c|c|c|c|c|c|}
\hline \multirow{2}{*}{$\begin{array}{l}\text { Condition } \\
\text { Asthma }\end{array}$} & \multirow[t]{2}{*}{ Age } & \multirow[t]{2}{*}{ Recruited from } & \multirow[t]{2}{*}{$\begin{array}{l}\text { Invited } \\
\text { to participate }\end{array}$} & \multicolumn{2}{|c|}{$\begin{array}{l}\text { Informed } \\
\text { consent }\end{array}$} & $\begin{array}{l}\text { Returned 1st } \\
\text { questionnaire }\end{array}$ & \multicolumn{2}{|c|}{$\begin{array}{l}\text { Returned } 2 \text { nd } \\
\text { questionnaire }\end{array}$} \\
\hline & & & & Maastri & icht & & & \\
\hline & $7-12$ & & 54 & 40 & $(74 \%)$ & $(74 \%)$ & 35 & $(65 \%)$ \\
\hline & $12-18$ & & 32 & 16 & $(50 \%)$ & $16(50 \%)$ & 12 & $(38 \%)$ \\
\hline \multirow[t]{3}{*}{ Diabetes } & \multicolumn{8}{|c|}{ Outpatients' clinics University Hospital Maastricht and Hospital Gooi-Noord } \\
\hline & $7-12$ & & 50 & 32 & $(64 \%)$ & $26(52 \%)$ & 24 & $(48 \%)$ \\
\hline & $12-18$ & & 74 & 39 & $(53 \%)$ & $34 \quad(46 \%)$ & 33 & $(45 \%)$ \\
\hline \multicolumn{2}{|c|}{ Rheumatic disorder } & \multicolumn{7}{|c|}{ Outpatients' clinic University Hospital Maastricht } \\
\hline & $7-12$ & & 37 & 11 & $(30 \%)$ & $(24 \%)$ & 9 & $(24 \%)$ \\
\hline & $12-18$ & & 41 & 13 & $(32 \%)$ & $(34 \%)$ & 13 & $(32 \%)$ \\
\hline \multirow[t]{2}{*}{ SLH disorder } & $7-12$ & \multicolumn{7}{|c|}{ Outpatients' clinic University Hospital Maastricht and special primary school } \\
\hline & $12-18$ & & 9 & 5 & $(56 \%)$ & $(56 \%)$ & 4 & $(44 \%)$ \\
\hline Total & & & & & & & & \\
\hline (Parent form) & $7-12$ & & 212 & 121 & $(57 \%)$ & $(53 \%)$ & 99 & $(47 \%)$ \\
\hline (Child form) & $12-18$ & & 156 & 73 & $(47 \%)$ & $69 \quad(44 \%)$ & 62 & $(40 \%)$ \\
\hline
\end{tabular}

SLH disorder $=$ Speech/language/hearing disorder 


\section{Baseline statistics}

Statistical descriptives (mean, standard deviation, $\mathrm{N}$ ) on the baseline questionnaire are presented in table 2 . On average, the scores were very high. The average VAS score was 83.04. Overall, the means were the highest among the children with diabetes and the lowest among the children with a rheumatic disorder.

Feasibility

In total, 21 parents and their children aged 7-12 years of age, and 8 children aged 12-18 years of age included comments on the questionnaires. These comments varied from statements on the children's' recent health state, to comments on the items in the questionnaire.

There was only one comment specifically about the EQ-5D. This was a comment by a parent with regard to the EQ-5D descriptive system who said that the item 'Pain/discomfort' was not specific enough. The answer 'My child has pain and does not feel good/sick' is difficult to answer if a child is in pain, but does not feel sick. In the total baseline group of 182 children only two of the five item scores were missing in the EQ$5 \mathrm{D}$. Both missing scores concerned the item 'Pain/discomfort' which were supposed to be answered by parents with children aged 7-12 years ( 1 asthma; 1 rheumatic disorder). The VAS was not completed by 16 participants (9\%). Of these percentage-wise the highest number of missing scores occurred among the children with a speech/language/hearing disorder (7 out of a total of 36).

With regard to the TAQCOL two of the parents of children with a speech/language/hearing disorder made comments on the question about "Going to school on their own" (scale: Autonomy), since most children were being picked up by bus. Some parents experienced this as being very autonomous and others thought this was not autonomous at all as other children go on foot or by bike. Furthermore, the TACQOL questions refer to the children's health status as experienced in the last few weeks. At least five questions were on the children's school activities which turned out to be difficult to answer if children were in the middle of their summer holidays. Three children ( 1 of 16 years old with diabetes and 2 children of 15 and 16 years old with a rheumatic disorder) added that they found the question about "Being asked by other children to play with them" too childish because of their age. That was also the reason why one child with a rheumatic disorder did not answer the question. In total, 6 TACQOL scale scores were missing. In this case too percentage-wise the highest number of missing scores occurred among the children with a speech/language/hearing disorder (3 children with 2 missing scores and 1 child with 3 missing scores). 


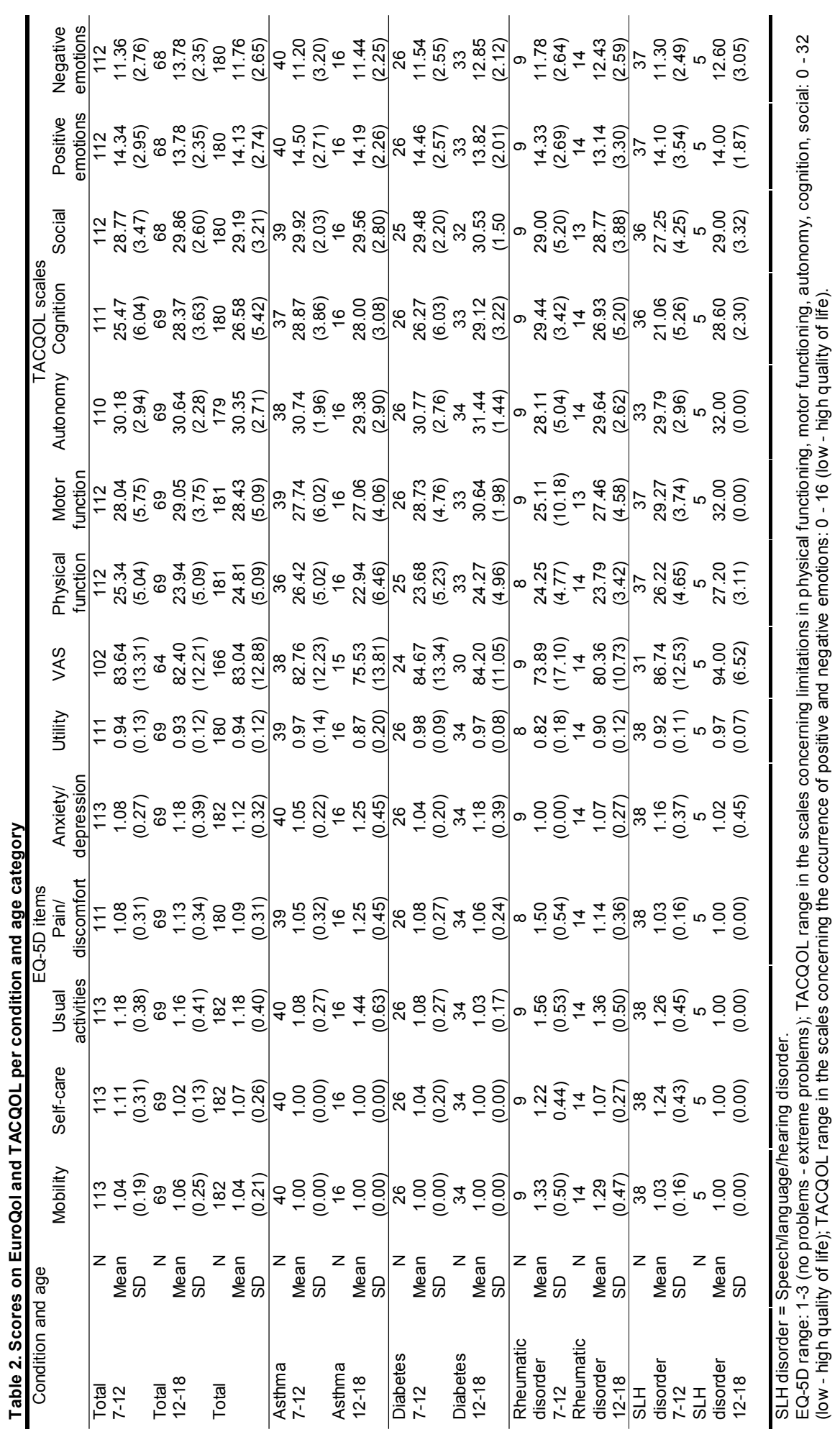




\section{Construct validity}

The baseline Spearman rank correlation coefficients of the EQ-5D, the EQ-VAS, the EQ5D utility (global score) and the TACQOL scales are presented in table 3 . On average low till moderate correlations were found between both questionnaires. In the total group the highest correlation (.566) was found between the EQ-5D utility and the 'Autonomy' scale of the TACQOL. Also, the EQ-5D item 'Usual Activities' and the scale 'Autonomy' (.531) and 'Motor functioning' (-.456) correlated high. Except for the correlations between the TACQOL scales 'Positive/negative emotions', 'Social' and 'Cognition' with the EQ-5D item 'Mobility', the correlation coefficients were statistically significant.

Compared to children aged 12-18 years, the EQ-5D item 'Self-care' correlated somewhat higher with all the TACQOL scales in children aged $7-12$ years.

\section{Discriminant power}

The stacked bars in figure 1 and the boxplot in figure 2 illustrate the discriminant power of the EQ-5D and the TACQOL scales. The EQ-5D discriminated the most between the children with a rheumatic disorder and the children with the other conditions. More specifically, in the group of children with a rheumatic disorder slightly more problems were reported on the items 'Mobility', 'Usual activities', and 'Pain/discomfort' than in the other groups.

With regard to the TACQOL scales the scales 'Positive and negative emotions' in particular did not discriminate between the conditions. The scale 'Motor functioning' differed in the children with a rheumatic disorder and the scale 'Cognition' was lower in the children with a speech/language/hearing disorder, as compared to the other groups.

Test-retest reliability

Table 4 provides intra-class correlation coefficients in the repeated scores of the EQ-5D items and the TACQOL scales.

The ICCs varied from -0.125 to 1.000 . With regard to utility the lowest ICC was found among the children with asthma and the highest ICC among the children with a rheumatic disorder. In the Bland Altman Plot in figure 3 the differences are plotted against the averages of the repeated measurements. The plot demonstrates that in patients with moderate VAS and Utility scores the within case variability is higher, and consequently the test-retest reliability lower. The ICCs for the subset of children who experienced no health change between the two questionnaires differed from the total population. The largest differences in the ICCs showed an upward tendency and 


\section{Chapter 5}

Table 3. Spearman correlations between scores on EQ-5D items (columns) and TACQOL scales (rows)

\begin{tabular}{|c|c|c|c|c|c|c|c|}
\hline$(163 \leq N \leq 181)$ & Mobility & Self-care & $\begin{array}{c}\text { Usual } \\
\text { activities }\end{array}$ & $\begin{array}{c}\text { Pain/ } \\
\text { discomfort }\end{array}$ & $\begin{array}{c}\text { Anxiety/ } \\
\text { depression }\end{array}$ & Utility & VAS \\
\hline \multicolumn{8}{|l|}{ Total } \\
\hline Physical function & -.089 & -.050 & $-.185^{*}$ & $-.302^{\star *}$ & $-.344^{* *}$ & $.327^{* *}$ & $.380^{* *}$ \\
\hline Motor function & $-.293^{* *}$ & $-.220^{* *}$ & $-.456^{* *}$ & $-.352^{\star *}$ & $-.150^{*}$ & $.481^{* *}$ & $.355^{* *}$ \\
\hline Autonomy & $-.327^{\star *}$ & $-.332^{\star \star}$ & $-.531^{* *}$ & $-.359^{\star *}$ & $-.164^{*}$ & $.566^{\star *}$ & $.377^{* *}$ \\
\hline Cognition & -.059 & $-.264^{\star *}$ & $-.231^{* *}$ & $-.153^{*}$ & $-.257^{* *}$ & $.335^{\star \star}$ & $.178^{*}$ \\
\hline Social & -.074 & $-.293^{\star \star}$ & $-.298^{* *}$ & $-.235^{\star *}$ & $-.301^{* *}$ & $.412^{\star *}$ & $.208^{* *}$ \\
\hline Positive emotions & -.066 & -.100 & $-.190^{*}$ & $-.270^{\star *}$ & $-.403^{* *}$ & $.382^{* *}$ & $.229^{* *}$ \\
\hline Negative emotions & .033 & $-.210^{\star *}$ & $-.269^{\star *}$ & $-.223^{* *}$ & $-.240^{\star *}$ & $.305^{\star *}$ & $.195^{*}$ \\
\hline \multicolumn{8}{|l|}{$7-12$ years } \\
\hline Physical function & -.095 & -.112 & $-.222^{*}$ & $-.350^{* *}$ & $-.301^{* *}$ & $.280^{* *}$ & $.417^{* *}$ \\
\hline Motor function & $-.281^{* *}$ & $-.227^{*}$ & $-.443^{* *}$ & $-.416^{\star *}$ & -.126 & $.432^{* *}$ & $.320^{* *}$ \\
\hline Autonomy & $-.323^{* *}$ & $-.377^{\star *}$ & $-.540^{* *}$ & $-.448^{\star *}$ & $-.224^{*}$ & $.602^{* *}$ & $.334^{* *}$ \\
\hline Cognition & .006 & $-.239^{*}$ & $-.239^{*}$ & -.100 & $-.308^{* *}$ & $.291^{* *}$ & .105 \\
\hline Social & -.234 & -.377 & -.371 & -.235 & -.215 & .423 & .285 \\
\hline Positive emotions & -.103 & -.167 & $-.288^{* *}$ & -.182 & $-.346^{\star *}$ & $.362^{* *}$ & $.220^{*}$ \\
\hline Negative emotions & -.070 & $-.251^{* *}$ & $-.289^{* *}$ & $-.217^{\star}$ & $-.253^{* *}$ & $.302^{* *}$ & $.236^{*}$ \\
\hline \multicolumn{8}{|l|}{$12-18$ years } \\
\hline Physical function & -.075 & .046 & -.122 & -.233 & $-.385^{\star *}$ & $.382^{* *}$ & $.318^{*}$ \\
\hline Motor function & $-.327^{* *}$ & -.217 & $-.480^{\star *}$ & $-.284^{*}$ & -.213 & $.572^{\star *}$ & $.427^{* *}$ \\
\hline Autonomy & $-.353^{\star *}$ & -.231 & $-.520^{* *}$ & $-.252^{*}$ & -.119 & $.519^{\star *}$ & $.460^{* *}$ \\
\hline Cognition & -.182 & -.207 & $-.241^{*}$ & $-.373^{\star *}$ & $-.372^{* *}$ & $.533^{* *}$ & $.431^{* *}$ \\
\hline Social & .097 & .023 & -.193 & $-.281^{*}$ & $-.469^{* *}$ & $.440^{* *}$ & .111 \\
\hline Positive emotions & .020 & .041 & .002 & $-.367^{\star *}$ & $-.474^{\star *}$ & $.401^{* \star}$ & .227 \\
\hline Negative emotions & .146 & .013 & $-.277^{\star}$ & $-.294^{*}$ & $-.317^{\star *}$ & $.364^{* *}$ & .160 \\
\hline
\end{tabular}

occurred in the item 'Usual activities', the utility score and the VAS. In the subset of children who stated that they had experienced a health change between the two questionnaires the correlations were lower in the items 'Pain/discomfort', 'Anxiety/depression', the utility score, and the VAS, compared to the correlations in the total group. Furthermore, the correlation was higher in the item 'Usual activities' and the same in the item 'Self-care'. 
Using EQ-5D in children with a chronic condition

Table 4. EQ-5D test-retest intra-class correlation coefficients

\begin{tabular}{|c|c|c|c|c|c|c|c|c|}
\hline Condition and age & & & & & $E Q-5 D$ iter & & & \\
\hline & & Mobility & Self-care & $\begin{array}{c}\text { Usual } \\
\text { activities }\end{array}$ & $\begin{array}{c}\text { Pain/ } \\
\text { discomfort }\end{array}$ & $\begin{array}{c}\text { Anxiety/ } \\
\text { depression }\end{array}$ & Utility & VAS \\
\hline Total 7-12 & $\mathrm{N}$ & 98 & 97 & 98 & 96 & 98 & 95 & 84 \\
\hline ICC test-retest & & 0.582 & 0.713 & 0.411 & 0.521 & 0.351 & 0.573 & 0.618 \\
\hline Total $12-18$ & $\mathrm{~N}$ & 62 & 62 & 62 & 61 & 62 & 61 & 55 \\
\hline ICC test-retest & & 0.548 & 1.000 & 0.346 & 0.281 & 0.624 & 0.435 & 0.589 \\
\hline Total & $\mathrm{N}$ & 160 & 159 & 160 & 157 & 160 & 156 & 139 \\
\hline ICC test-retest & & 0.565 & 0.748 & 0.385 & 0.586 & 0.495 & 0.514 & 0.608 \\
\hline Subset ICC test- & $\mathrm{N}$ & - & 68 & 69 & 68 & 69 & 67 & 58 \\
\hline $\begin{array}{l}\text { retest no health } \\
\text { change }\end{array}$ & & - & 0.734 & 0.627 & 0.485 & 0.357 & 0.656 & 0.755 \\
\hline Subset ICC test- & $\mathrm{N}$ & - & 20 & 20 & 19 & 20 & 20 & 19 \\
\hline $\begin{array}{l}\text { retest health } \\
\text { change }\end{array}$ & & - & 0.782 & 0.487 & -0.125 & -0.053 & 0.257 & 0.121 \\
\hline Asthma 7-12 & $\mathrm{N}$ & - & - & 35 & 34 & 35 & 32 & 33 \\
\hline ICC test-retest & & - & - & 0.192 & 0.476 & 0.358 & 0.384 & 0.404 \\
\hline Asthma 12-18 & $\mathrm{N}$ & - & - & 12 & 12 & 12 & 11 & 12 \\
\hline ICC test-retest & & - & - & 0.051 & 0.407 & 0.372 & 0.186 & 0.196 \\
\hline Diabetes 7-12 & $\mathrm{N}$ & - & - & 24 & 24 & 24 & 21 & 24 \\
\hline ICC test-retest & & - & - & 0.488 & 0.000 & 0.000 & 0.617 & 0.085 \\
\hline Diabetes $12-18$ & $\mathrm{~N}$ & - & - & 33 & 32 & 33 & 27 & 32 \\
\hline ICC test-retest & & - & - & 1.000 & 0.467 & 0.675 & 0.581 & 0.577 \\
\hline Rheum. dis. 7-12 & $\mathrm{N}$ & 9 & 9 & 9 & 8 & - & 9 & 8 \\
\hline ICC test-retest & & 0.789 & 1.000 & 0.158 & 1.000 & - & 0.837 & 0.941 \\
\hline Rheum. dis. 12-18 & $\mathrm{N}$ & 13 & 13 & 13 & 13 & 13 & 13 & 13 \\
\hline ICC test-retest & & 0.424 & 1.000 & 0.278 & 0.000 & 1.000 & 0.669 & 0.493 \\
\hline SLH disorder 7-12 & $\mathrm{N}$ & - & 30 & 30 & - & 30 & 22 & 30 \\
\hline ICC test-retest & & - & 0.708 & 0.558 & - & 0.650 & 0.607 & 0.714 \\
\hline SLH disorder $12-18$ & $\mathrm{~N}$ & - & - & - & - & - & - & 4 \\
\hline ICC test-retest & & - & - & - & - & - & - & 0.765 \\
\hline
\end{tabular}

Rheum. dis. = rheumatic disorder; SLH disorder = Speech/language/hearing disorder; - = analyses could not be performed due to low variance; Subset ICC test-retest no health change = ICC test-retest for the subset of children who did not experience health changes between the two questionnaires; Subset ICC test-retest health change = ICC test-retest for the subset of children who did experience health changes between the two questionnaires. 


\section{Chapter 5}

\section{Conclusion}

The aim of the study was to explore the use of the EQ-5D in children with chronic conditions. This was investigated among children with asthma, diabetes, a rheumatic disorder, and a speech/language/hearing disorder. The feasibility, construct validity and discriminant power were compared with a generic questionnaire for children (TACQOL). Furthermore, the 2-week test-retest reliability of the EQ-5D child version was assessed. As expected, the feasibility of the EQ-5D is good. Only one comment was given that was especially about the EQ-5D. Also, missing scores were minimal.

The low to moderate Spearman rank correlations suggest that the EQ-5D and TACQOL seem to be measuring different concepts. Remarkably even the corresponding concepts did not correlate high. The TACQOL seems to have an additional value, especially with regard to cognitive functioning.

The discriminant power of the items in the EQ-5D is low and the highest differences were found between the children with a rheumatic disorder and the children with the other conditions. Contrary to our expectations, patients with asthma stated that they had no more problems with their usual activities nor did they experience more pain/discomfort than children with diabetes or a speech/language/hearing disorder. In contrast to the TACQOL scales, the EQ-5D was not able to distinguish children with a speech/language/hearing disorder from children with other conditions.

In the subset of children who experienced no health change between the questionnaires the test-retest reliability is moderate to high. The low ICCs in children who did experience a health change demonstrates that the generic quality of life in this population varied substantially over time, even with a 15 day interval. The consequence of this finding is that large sample sizes are needed in children with a variable disease status. Preferably the utility of these children should be frequently monitored.

The feasibility seemed the lowest among children with a speech/language/hearing disorder. Possibly this is caused by additional problems, such as a poor ability to read. In this study the TACQOL was completed by children aged 7-18 years, while actually the TACQOL was developed for children up to 15 years of age. This resulted in comments on the suitability of the questionnaire for juveniles and possibly an adult version might have been more appropriate for these children. Furthermore, school holidays led to confusion with regard to some school-related questions. This period should therefore be avoided as far as possible, or additional instructions should be provided if intending to conduct the TACQOL.

With regard to the items 'Mobility' and 'Self-care' ceiling effects were observed, as the main answer was 'Having no problems' and variance was low. This was especially the 
case in children with asthma and diabetes. For that reason some analyses could not be performed for these children. A possible explanation for these ceiling effects might be that these children are adapted to their condition and have already developed a different way of dealing with their health problems. Furthermore, the very high quality of life on average in the study populations may have lowered the discriminant power.

The low construct validity may be caused by the differences between the TACQOL and the EQ-5D. The TACQOL is a population specific questionnaire, while the EQ-5D is a generic questionnaire. Also, the EQ-5D and the TACQOL have different points of departure in measuring quality of life. The aim of the EQ-5D is to measure the degree that quality of life is present and that the aim of the TAQCOL is to measure the degree that quality of life is absent. Furthermore, compared to the children aged 12-18 years, the EQ-5D item 'Self-care' correlated somewhat higher with the item 'Anxiety/depression' and somewhat lower with all the TACQOL scales among the children aged 7-12 years. Nevertheless these differences were very small.

The results of the 2-week test-retest analyses imply that the EQ-5D and the TAQCOL are less suitable for children in which the course of the condition seems to be variable. Since the study population represented children with possibly a large variation in the severity of their chronic condition, it is important that the findings are replicated in a more strictly defined research population.

A number of studies have performed test-retest analyses to explore the reliability of the EQ-5D in adults with different conditions. For instance, a moderate ICC (overall score 0.46 ) was found after a 5-week test-retest in an adult population with rheumatoid arthritis (12). A higher ICC of 0.64 was found in another study after a 2-week test-retest among adult patients with a rheumatic disease (13). Consequently, a shorter test-retest period appears to increase the correlation rapidly. In adults with an inflammatory bowel disease an ICC of 0.89 was found in patients with an unchanged health status after a 2-week test-retest (14). The ICC in adult patients with a chronic obstructive pulmonary disease was 0.73 after 2 weeks (15). Overall, the correlations in the studies among adults with a 2-week test-retest seemed higher than the correlations among children, as found in our study. Generic quality of life is influenced by a number of factors which may not all be directly associated with the condition. It would be interesting to examine whether other factors, such as for instance quarrels between the parents or with friends or small traumas play a different role in the perception of quality of life in children as compared to adults.

As the health state of children with a chronic condition is changeable over time, the competence to complete questionnaires may have changed between the first and the 


\section{Chapter 5}

second questionnaire. For that reason a question about the administration method was included. In total, 136 participants (71 parents and 55 children) of the 161 participants (99 parents and 62 children) who completed both questionnaires answered in both questionnaires if their administration method was with or without help. Of these 71 parents with children aged 7-12 years, 22 parents changed their administration method. Of the 55 children aged 12-18 years, 9 children changed their administration method at the retest. Analyses show that the exclusion of children who changed their administration method leads to an increase of the ICC with regard to the EQ-5D item 'Anxiety/depression' and the utility. As all things should be equal to obtain reliability (16), we suggest requesting the same administration method during a test-retest analysis. The increase of the correlation coefficients, after excluding patients who experienced differences in their health status, also emphasises the importance of retrieving more information with regard to the participant's health status, changes in personal matters or environment in future test-retest analyses.

Remarkably the items 'pain/discomfort' and 'anxiety/depression' are not formulated the same in the child version as in the adult version of the EQ-5D. As illustrated in the appendix the word 'and' is replaced by the word 'or'. This may lead to confusion as the answer 'I am not anxious and I am cheerful' (child version) can be differently interpreted than the answer 'I am not anxious or sad' (adult version).

There is a growing demand for child health assessment tools (2). These tools differ from adult health assessment tools, for instance due to different ages, cognitive capacity and developmental stage. Generic preference-based instruments for children are very important, since they are used for economic evaluations on which decisions are based for paediatric health interventions. There is currently still extensive variation in the methods used by researchers (17). Economic evaluation in health care often involves cost-utility analysis, a method based on the cost-effectiveness criterion of monetary units per QALY. The EQ-5D is a well known instrument for generating these QALY scores. Utility scores derived from the EQ-5D are weighted irrespective of who those QALYs go to and how they are distributed across society (18). The social aspect is independent of age, while in real life some people wish to give more priority to for instance children compared to adults. Another reason which complicates QALY measurement is that in children different attributes may be relevant, for example, autonomy and family relationships (17). It appears that multi-attribute utility instruments are not simply suitable for children as a matter of course. For these reasons caution should be taken when using EQ-5D derived QALY scores in child populations. For the future it is recommended that research is done to investigate the extent to which preferences change if age is 
taken into account. Depending on the outcomes, this could eventually lead to the development of generic preference-based utility functions for children. Furthermore, this instrument should take into account the fact that children adapt to their situation and the children's view of their condition (5) and may have additional value when measuring quality of life in children.

In conclusion, the EQ-5D can be easily conducted among children and parents of children with chronic conditions. The EQ-5D may be less suitable for children who experience cognitive problems (as among children with a speech/language/hearing disorder in this study) and if the course of a condition is variable. The EQ-5D seems the most suitable for children with a chronic physical condition. Although the ICCs are lower than those observed among adults, the EQ-5D child version appears to be reliable for children with a stable health status. 


\section{Chapter 5}

\section{References}

1. Drummond MF, Sculpher MJ, Torrance GW, O'Brien BJ, Stoddart GL. Methods for the economic evaluation of health care programmes. 3rd ed. Oxford: Oxford University Press; 2005.

2. Spilker B (ed.) Quality of life and pharmacoeconomics in clinical trials. 2nd ed. Philidelphia: Lippencott-Raven Publishers; 1996.

3. Brooks R. EuroQol: the current state of play. Health Policy. 1996; 37:53-72.

4. Stolk EA, Busschbach JJ, Vogels T. Performance of the EuroQol in children with imperforate anus. Quality Life Res. 2000; 9:29-38.

5. Eiser C, Morse R. Quality-of-life measures in chronic diseases of childhood. Health Technol Assess. 2001; 5:1-157.

6. World Health Organization (WHO) [homepage on the internet]. Chronic diseases and health promotion; 2007. Available from: http://www.who.int/chp/en/.

7. Baan CA, Hutten JB, Rijken PM, (red.) Afstemming in de zorg: een achtergrondstudie naar de zorg voor mensen met een chronische aandoening [in Dutch]. RIVM rapport 282701005/2003.

8. Theunissen NC, Vogels AG, Koopman HM. The proxy problem: child report versus parent report in healthrelated quality-of-life research. Qual Life Res. 1998; 7:387-97.

9. Verrips $\mathrm{GH}$, Vogels AG, Koopman HM. Measuring health-related quality-of-life in a child population. Eur J Public Health. 1999; 9:188-93.

10. Vogels T, Verrips GH, Verloove-Vanhorick SP et al. Measuring health-related quality-of-life in children: the development of theTACQOL parent form. Qual Life Res. 1998; 7:457-65.

11. Vogels T, Verrips GH, Koopman HM, Theunissen NC, Fekkes M, Kamphuis RP. TACQOL Manual: parent form and child form, Leiden Center for Child Health And Pediatrics LUMC-TNO; 2000.

12. Marra CA, Rashidi AA, Guh D, et al. Are indirect utility measures reliable and responsive in rheumatoid arthritis patients? Qual Life Res. 2005; 14:1333-44.

13. Luo N, Chew LH, Fong KY, et al. A comparison of the EuroQol-5D and the Health Utilities Index mark 3 in patients with rheumatic disease. J Reumatol. 2003; 30:2268-74.

14. Konig HH, Ulshofer A, Gregor M, et al. Validation of the EuroQol questionnaire in patients with inflammatory bowel disease. Eur J Gastroenterol Hepatol. 2002; 14:1205-15.

15. Stavem K, Reliability, validity and responsiveness of two multiattribute utility measures in patients with chronic obstructive pulmonary disease. Qual Life Res. 1999; 8:45-54.

16. Hays RD, Anderson R, Revicki D. Psychometric considerations in evaluating health-related quality of life measures. Qual Life Res. 1993; 2:441-9.

17. Griebsch I, Coast J, Brown J. Quality-adjusted life-years lack quality in pediatric care: a critical review of published cost-utility studies in child health. Pediatrics. 2005; 115:e600-14.

18. Dolan P, Shaw R, Tsuchiya A, Williams A. QALY maximisation and people's preferences: a methodological review of the literature. Health Econ. 2005; 14:197-208. 


\section{Appendix}

EQ-5D proxy and child version translated by the author

Proxy version EQ-5D

Indicate which statement best describes the health state of your child today.

Mobility / walking about

My child has no problems walking about

My child has some problems in walking about

My child has to lie in bed all day

Take care of oneself (e.g. going to the toilet, washing oneself or getting dressed)

My child has no problems with taking care of her/himself

My child has some problems with taking care of her/himself

My child is unable to take care of her/himself

Usual activities (e.g. playing, helping around the house, going to school)

My child has no problems with performing her/his usual activities

My child has some problems with performing her/his usual activities

My child is unable to perform her/his usual activities

Pain / feeling ill

My child has no pain and does not feel ill

My child has pain and does feel ill

My child has a lot of pain and feels very ill

Mood

My child is not anxious and is cheerful

My child is a little anxious and hardly in the mood to do anything

My child is afraid and is in no mood to do anything

Child version EQ-5D

Indicate which statement best describes your health state today.

Mobility / walking about

I have no problems walking about

I have some problems walking about

I have to lie in bed all day

Self-care

I have no problems washing or dressing myself

I have some problems washing or dressing myself

I am unable to wash or dress myself

Usual activities (e.g. playing, helping with housekeeping, going to school)

I have no problems performing my usual activities

I have some problems performing my usual activities

I am unable to perform my usual activities

Pain / feeling ill

I have no pain and feel very healthy

I have no pain and do not feel well

I have a lot of pain and feel very il

Mood

I am not anxious and I am cheerful

$\mathrm{O}$

I am a little anxious and hardly in the mood to do anything

$\mathrm{I}$ am afraid and in no mood to do anything 
Cost-effectiveness of a nurse-led telemonitoring intervention based on peak expiratory flow measurements in asthmatics: results of a randomised controlled trial

BMC Cost Eff Resour Alloc 2007, 5:10

DCM Willems MA Joore JJE Hendriks EFM Wouters JL Severens

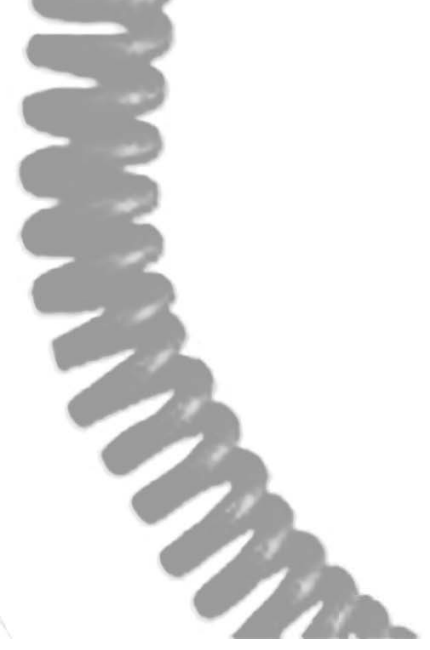




\section{Chapter 6}

\section{Abstract}

Background: Asthma is a chronic lung disease in which recurrent asthma symptoms create a substantial burden to individuals and their families. At the same time the economic burden associated with asthma is considerable.

Methods: The cost-effectiveness study was part of a single centre prospective randomised controlled trial comparing a nurse-led telemonitoring programme to usual care in a population of asthmatic outpatients. The study included 109 asthmatic outpatients (56 children; 53 adults). The duration of follow-up was 12 months, and measurements were performed at baseline, 4 , 8, and 12 months. Patients were asked to transfer their monitor data at least twice daily and by judging the received data and following a stepwise intervention protocol a nurse was able to act as the main care provider in the intervention group. In both groups the EQ-5D and the SF-6D were used to obtain estimates of health state utilities. One year health care costs, patient and family costs, and productivity losses were calculated. The mean incremental costs were weighted against the mean incremental effect in terms of QALY.

Results: The study population generally represented mild to moderate asthmatics. No significant differences were found between the groups with regard to the generic quality of life. Overall, the mean health care costs per patient were higher in the intervention group than in the control group. The intervention costs mainly caused the cost difference between the groups. The intervention costs the society $€ 31,035$ /QALY gained with regard to adults and with regard to children $€ 59,071$ /QALY gained.

Conclusions: If the outcome is measured by generic quality of life the nurse-led telemonitoring programme is of limited cost-effectiveness in the study population. From the societal perspective the probability of the programme being cost-effective compared to regular care was $85 \%$ at a ceiling ratio of $€ 80,000 /$ QLY gained among the adults and $68 \%$ among the children. Decreases in the price of the asthma monitor will substantially increase the probability of the programme to be cost-effective. 


\section{Background}

Asthma is a chronic lung disease from which worldwide 300 million people suffer. Among children it is even the most common chronic disease. Recurrent asthma symptoms create a substantial burden to individuals and their families and lead to restriction of an individuals' activities. Asthma can be successfully controlled with proper care which enables patients to enjoy good quality of life (1). In a continuous cycle therapy should be adjusted by assessment, treatment, and monitoring, depending on the patient's level of control (2). If asthma is not in control it can lead to increase in medical consumption and to school and work absenteeism. Consequently the economic burden associated with asthma is considerable (1). In the year 2000 the total costs for an asthma patient were on average $€ 312$ in the Netherlands. Medication costs were the largest cost component in the direct costs $(53 \%)$. Furthermore, the medication costs were estimated to triplicate by the year 2025 (3). A study performed in 2003 in the USA has concluded that caring for a severe asthmatic costs $\$ 12,813$ a year (approximately $€ 10,000$ ) (4). The largest components in the direct costs were medication (53\%) and hospital admissions (15\%). Since financial resources are scarce and technology is improving economic evaluations are helpful in making decisions among different health care interventions. This is particularly useful to decide if a new health care technology should be implemented. Economic evaluations present a comparative analysis of alternative courses of actions in terms of both their costs and consequences. The incremental cost-effectiveness ratio is defined as the difference in costs between two technologies divided by the difference in their benefits. The lower the incremental cost-effectiveness ratio, the more cost effective a technology (5).

Telemonitoring is a recent development in health care and offers new method to manage asthma. By the use of telemonitors, spirometry tests can continuously be monitored by transferring monitor data from the patient's home to a central database. This monitoring at a distance is very useful in the management of asthma, since asthma cannot be cured and the presence of asthma symptoms can change every day. In a recent literature review it was stated that long-term disease monitoring of patients at home stimulates cost-effectiveness in health care (6). Nurses increasingly play a part in telemonitoring and nurse-led telemonitoring programmes in chronic diseases are commonly considered cost-effective. By employing a nurse (practitioner) as the main care provider costs can be decreased to a minimum, while the quality of care stays intact (7). However, only a few studies have calculated costs or incremental costs-effectiveness ratios (8). To our knowledge no study has performed a cost-effectiveness analysis of a nurse-led telemonitoring intervention in asthmatic outpatients. The objective of this study was to 


\section{Chapter 6}

determine the cost-effectiveness of a nurse-led telemonitoring programme in asthmatic outpatients compared to regular care. The study protocol was approved by the appropriate ethics committee and informed consent was obtained from each participant.

\section{Methods}

Data source

The cost-effectiveness study was part of a single centre prospective randomised controlled trial comparing a nurse-led telemonitoring programme to usual care in a population of asthmatic outpatients. Randomisation took place on patient level after stratification by age (children aged 7 to 18 versus adults aged 18 years and older). The duration of follow-up was 12 months, and measurements were performed at baseline, 4 , 8 , and 12 months.

The study population consisted of asthmatic outpatients from the Medical Respiratory Department and the Department of Paediatrics at the University Hospital Maastricht in the Netherlands. Patients aged 7 and older with an asthma severity of stage I-III as described in the GINA guidelines were potentially eligible. The patients had to be competent to use an asthma monitor, and had to possess a household phone connection. Exclusion criteria were severe co-morbidity (such as cystic fibrosis or congenital lung abnormalities), since these patients frequently require outpatient visits. The inclusion and exclusion criteria were verified by the use of patient records. The power calculation for this study was based on improvement in asthma-specific quality of life, with a minimal important difference of 0.5 points on the 7-point overall scale in the Asthma Quality of Life Questionnaire (AQLQ) or the Paediatric Asthma Quality of Life Questionnaire (PAQLQ) (9-11). One-sided testing, a power of $80 \%$, and a significance level of 0.05 with a drop out percentage of $10 \%$ resulted in a minimal inclusion of 51 patients in the intervention group and 51 patients in the control group.

This study focuses on the cost-effectiveness analyses. The process evaluation and the effects of the main outcome parameters ((P)AQLQ) are described elsewhere (12).

\section{Comparators}

The control group received regular outpatient care. In case of stable asthma these patients received three to six monthly medical check ups by their lung specialist or paediatrician. In case of exacerbations the patients received additional care from their general practitioner and/or outpatient care. The intervention group used an asthma monitor at home, and had a hospital-based nurse practitioner (also referred to as the asthma nurse) as the main care provider. The monitor was a portable hand-held device 
with a matching modem. Patients were able to review their spirometry test results on the monitor screen. Transfers of the monitor data to the nurse practitioner's computer were performed by the patients by connecting the modem to the household phone. Patients were instructed to perform daily lung function tests (both in the morning and in the evening) and more often if they were having symptoms. Patients were asked to transfer the monitor data to the nurse practitioner every month and more frequently if they were having asthma symptoms. Patients with less serious symptoms were instructed to call the nurse practitioner during their programme participation. The nurse practitioner studied the data and classified the asthma following a stepwise intervention protocol. According to this protocol the nurse practitioner was allowed to decrease (after 3 months of stable asthma) or increase (if asthma was unstable) asthma medication by one step. In case of an exacerbation the nurse practitioner judged if the exacerbation was only an incident or more structural. If he concluded that this was structural the nurse practitioner had to increase the treatment by one therapy step as described in the protocol. If standard treatment of an exacerbation was not successful the nurse practitioner contacted the treating physician to decide whether or not a steroid course was necessary. In addition, according to the protocol every 3 months the medication could be changed ( 1 step up or down) by the nurse practitioner. If the PEF curve was within normal range and the symptoms were minimal the nurse practitioner asked the patients to decrease the treatment level by one step. A physician was only consulted if necessary. Following this procedure, the nurse practitioner could adjust or maintain the treatment due to the continuously monitoring.

\section{Effects}

Two multi-attribute utility instruments were used to obtain estimates of health state utilities: the EQ-5D and the SF-6D. Both instruments were administered at baseline, and at 4,8 , and 12 months follow-up.

The EQ-5D consists of a descriptive system and a visual analogue scale (13). The five questions in the EQ-5D classify persons into one of 243 health states. The commonly used scoring function for the EQ-5D is based on a British study (EQ-5D UK) with preferences derived by the time trade-off-method (TTO), in a representative sample of the UK population (14). The possible range of utility scores is from -0.59 to 1.00 . In the subgroup of children the EQ-5D child version as previously used by Stolk et al. (15) was used. Children aged 12 years and older completed this version themselves, for children younger than 12 years of age the parents or caregivers completed the child proxy version. 


\section{Chapter 6}

The SF-6D is derived from the SF-36 (16). The SF-6D instrument covers 6 domains, and describes 18,000 health states. Using a fractional factorial design, 249 health states were identified and valued by a representative sample of the UK general population using the standard gamble valuation method (17). An algorithm provided by Brazier and colleagues was used to construct the utilities. The utility scores range from 0.29 to 1.0. SF-6D utilities could only be obtained in the subgroup of adult participants because no child version is available.

Costs

Costs can be divided into health care costs, patient and family costs, productivity losses, and costs in other sectors (5). Health care costs included costs associated with hospital care, general practitioners and other health care professionals, prescribed medication, professional home care, and the intervention. Hospital care included day admissions, emergency room visits, surgical and diagnostic procedures, laboratory research, and outpatient visits. Patient and family costs consisted of the costs of over-the-counter medication, and informal care. Productivity losses include the costs due to productivity loss at paid and unpaid work. Costs in other sectors are the resources consumed in other sectors, such as volunteer work and nursing home care. These costs were absent in this study and therefore not included in the analysis. The volumes of hospital care were obtained from the hospital billing system of the University Hospital Maastricht. All other resource use was collected using a four weeks prospective cost diary completed at 1, 4, 8 and 12 months follow-up. Cost diaries have proven to be a successful means to gather information on health care resource use during a longer period (18). The data from each cost diary were interpolated (multiplied by 3 ) in order to obtain estimates of resource use during the entire one year follow-up period.

Unit prices from the Dutch manual for cost research were used if available (19). Costs associated with the loss of productivity at volunteer work or household activities were calculated using a shadow price of $€ 8.30$ per hour of absence. Costs from productivity loss at paid work were calculated according to the friction cost method (20). This method calculates productivity loss costs for the duration of the friction period. The friction period is the theoretical time needed to fill a vacancy as a result of illness. This method is recommended by the Dutch guidelines for pharmaco-economic research (21). Costs of school absenteeism were incorporated in a sensitivity analysis. This calculation was mainly based on data of the Ministry of Education, Culture and Science (22). The hours of school absenteeism were derived from the cost diaries. The costs associated with school absenteeism consisted of government costs and (voluntary) parental contribution 
and these costs depend on school type and class. The total costs were calculated by multiplying the hours of school absenteeism with the corresponding unit prices.

For the costs of the intervention a micro costing calculation was performed. The intervention costs consisted of costs of materials (the asthma monitor, and computer equipment), costs of personnel (the nurse practitioner), telephone costs, and travel costs. The price of the asthma monitor was $€ 476$, and the price of the modem $€ 1,428$. Depreciation over five years, with $4,5 \%$ interest, leads to annual costs of $€ 434$ per patient. The annual costs of an insurance for the equipment amounted to $€ 16$ per patient. The costs of the computer equipment the nurse practitioner used to receive and analyse the transferred data (a personal computer, software, monitor, and printer) amounted to $€ 1,150$. For 55 patients, depreciated over five years, with $4,5 \%$ interest, these costs equal $€ 5$ per patient per year. Other fixed costs were associated with the development and production of instruction material ( $€ 4$ and $€ 7$ per patient per year), and administrative tasks of the nurse practitioner ( $€ 7$ per patient per year). A continuous time registration of the activities (reviewing the lung function data the patients send in, adjusting the treatment plans, telephone contacts and house calls) of the nurse practitioner was performed. Based on the salary costs of a nurse practitioner ( $€ 44,700$ per year), and 1540 workable hours per year, the costs of the nurse practitioner were estimated to amount to $€ 29$ per hour. Costs of repair of the asthma monitor and modem were registered by the nurse practitioner.

Overhead costs were calculated over all direct material and personnel costs (35\%) (19). All costs were calculated for a period of one year, therefore discounting was not indicated. Costs are presented in euro for the year 2002.

\section{Statistical analyses}

Analyses were performed by the intention-to-treat principle. Data imputations for missing values were carried out consecutively in three steps. Firstly, overall mean scores at baseline substituted missing baseline scores. Secondly, missing scores between two valid scores measured in time were individually interpolated. Thirdly, remaining missing values were imputed by Last Value Carried Forward procedure. If data was normally distributed analysis on the resulting complete data was done by repeated measures ANCOVA using 'time' as a fourth category within-patients factor and both the experimental factor (control/intervention) as well as type of patient (child/adult) as dichotomous between factors. The full model ANCOVA allowed for testing of first and second order interactions between the covariate (baseline scale scores) and both factors. 


\section{Chapter 6}

Adjustments for baseline differences in health state utility for follow-up took place. In the intervention group, for children and adults apart, the utility scores during the follow-up measurements were corrected with the mean difference in baseline utility between the intervention group and the control group. All significant test results involved 2-tailed probabilities with alpha set at 0.05 . Analyses were performed according to the intentionto-treat principle. Statistical analysis was performed using SPSS, version 12.0.

\section{Cost-effectiveness analysis}

The time horizon of the cost-effectiveness analyses was 1 year. The cost-effectiveness analysis was conducted from both the health care and the societal perspective. Adults and children were analysed separately. In the analyses from both perspectives the mean incremental health care costs were weighted against the mean incremental effect in terms of quality adjusted life years (QALY). Since no mortality occurred the QALY was calculated for each patient by multiplying each of the four measured utility values with 4 months. This calculation was performed by the use of the EQ-5D utility (adults and children) and the SF-6D utility (adults only). To obtain the incremental QALY multiple regression analysis was applied to control for the differences in baseline utility. To get insight into the uncertainty around the incremental cost-effectiveness ratio (ICER) nonparametric bootstrap simulations were conducted (23). Bootstrapping was performed on both the incremental regression based QALY (based on both the EQ-5D utility and the SF-6D utility) versus both the incremental health care costs and the incremental societal costs. In the bootstrap simulation 5000 random samples of cost-effect pairs, of equal size of the original sample, were selected with replacement. The scatter plots represent points of which each signifies the incremental cost-effectiveness ratio of one iteration of the bootstrap simulation. From a decision-makers point of view, the probability that a new treatment is cost-effective varies depending on what society is prepared to pay per gain in effectiveness, the so-called ceiling ratio. In the Netherlands $€$ 80,000 /QALY gained has been mentioned (24). Another mentioned ceiling ratio is $€$ 40,000 /QALY gained. This is shown in cost-effectiveness acceptability curves.

\section{Sensitivity analyses}

One-way sensitivity analyses are performed to test for the impact of two cost components on the study results. In the first analysis the total asthma monitor costs including monitor, modem, batteries, and insurance (€ 450) were set to zero in the intervention group. In the second analysis the costs resulting from school absenteeism by children were included in the societal costs. 


\section{Results}

\section{Study population}

From patient records of the departments of Respiratory Medicine and Paediatrics of our hospital 274 potentially eligible asthmatic outpatients were identified and approached by letter. Eighteen patients were not eligible because of the absence of a house phone connection (7\%), and 147 patients refused to participate in the study (54\%). The most frequently reasons of the 40 adults and 26 children who registered their reason for not participation were: 'having no time' ( $29 \%$ adults; $14 \%$ children), 'being uninterested' ( $9 \%$ adults; $11 \%$ children), 'not experiencing asthma symptoms' ( $5 \%$ adults; $8 \%$ children), and 'finding participation too confronting' ( $5 \%$ adults; $2 \%$ children). In the end, $40 \%$ was included in the study. Between January 2003 and January 2004, 109 patients (53 adults and 56 children) were enrolled in the study. The characteristics of the intervention and control group appeared to be similar at baseline, except for baseline utility. The patient characteristics of the study population are presented in table 1.

Of the 109 participants, seven patients ( 5 intervention group; 2 control group) were lost to follow-up. In total five patients of the 55 patients in the intervention group were lost to follow-up (two adults and three children). In the control group two of the 54 patients were lost to follow-up (one adult and one child). The reason to stop participation was for one patient in the control group immigration, further all lost to follow-up occurred because the patients refused further participation. Reasons for these refusals were not given. In the intervention group four cases of loss to follow-up took place immediately after the baseline measurement, and one case occurred after the third measurement at eight months. In the control group one patient was lost to follow-up after the baseline measurement and one patient after the third measurement at eight months. 


\section{Chapter 6}

Table 1. Baseline characteristics of the patients by age and group

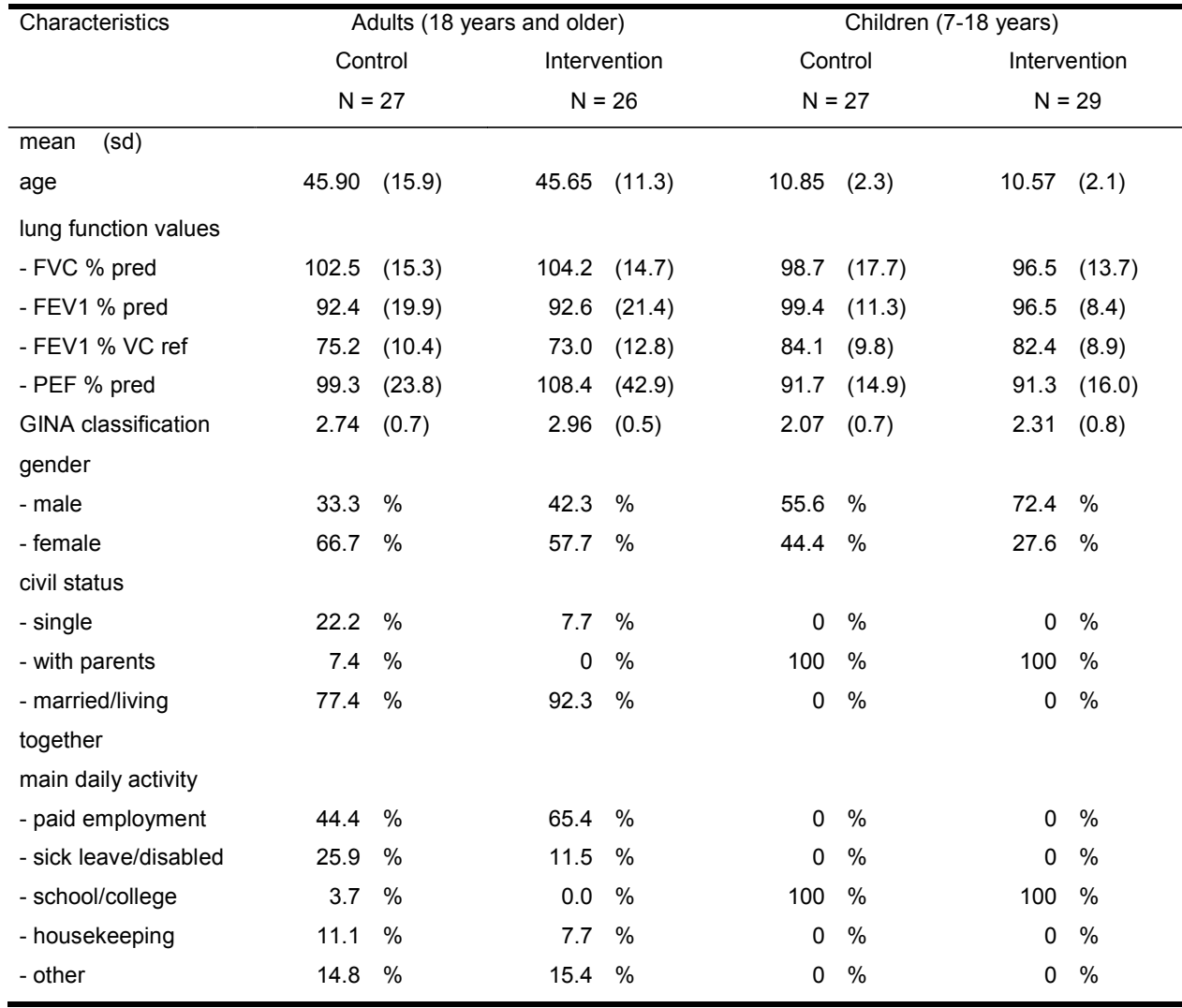

FVC \% pred = forced vital capacity expressed as a percentage of predicted; FEV1 \% pred = forced expiratory volume in 1 second expressed as a percentage of predicted; FEV1 \% VC ref = forced expiratory volume expressed as a percentage of predicted/vital capacity; PEF \% pred = peak expiratory flow expressed as a percentage of predicted. GINA classification based on prescribed medication; 1 = intermittent asthma, 2 = persistently mild asthma, 3 = persistently moderate asthma, 4 = persistently severe asthma.

\section{Effects}

Generic quality of life as measured with the EQ-5D descriptive part showed little to no problems at all measurements. The scores on the VAS indicated a moderate to good self perceived health status in both groups. The population utility scores were higher than the scores on the VAS. No differences between groups in time in the VAS scores were observed in the adults $(P=.638$; ANCOVA) or the children $(P=.521$; ANCOVA). See table $2 a$ and $2 b$.

Overall, the adults in the intervention group experienced fewer problems with regard to the items of the EQ-5D including the baseline measurement then the control group. In time, the domain scores all significantly improved in both groups. Between the groups in time no statistically significant differences were observed. SF-6D scores and utility were relatively high, and slightly higher in the intervention group at all measurements. The 


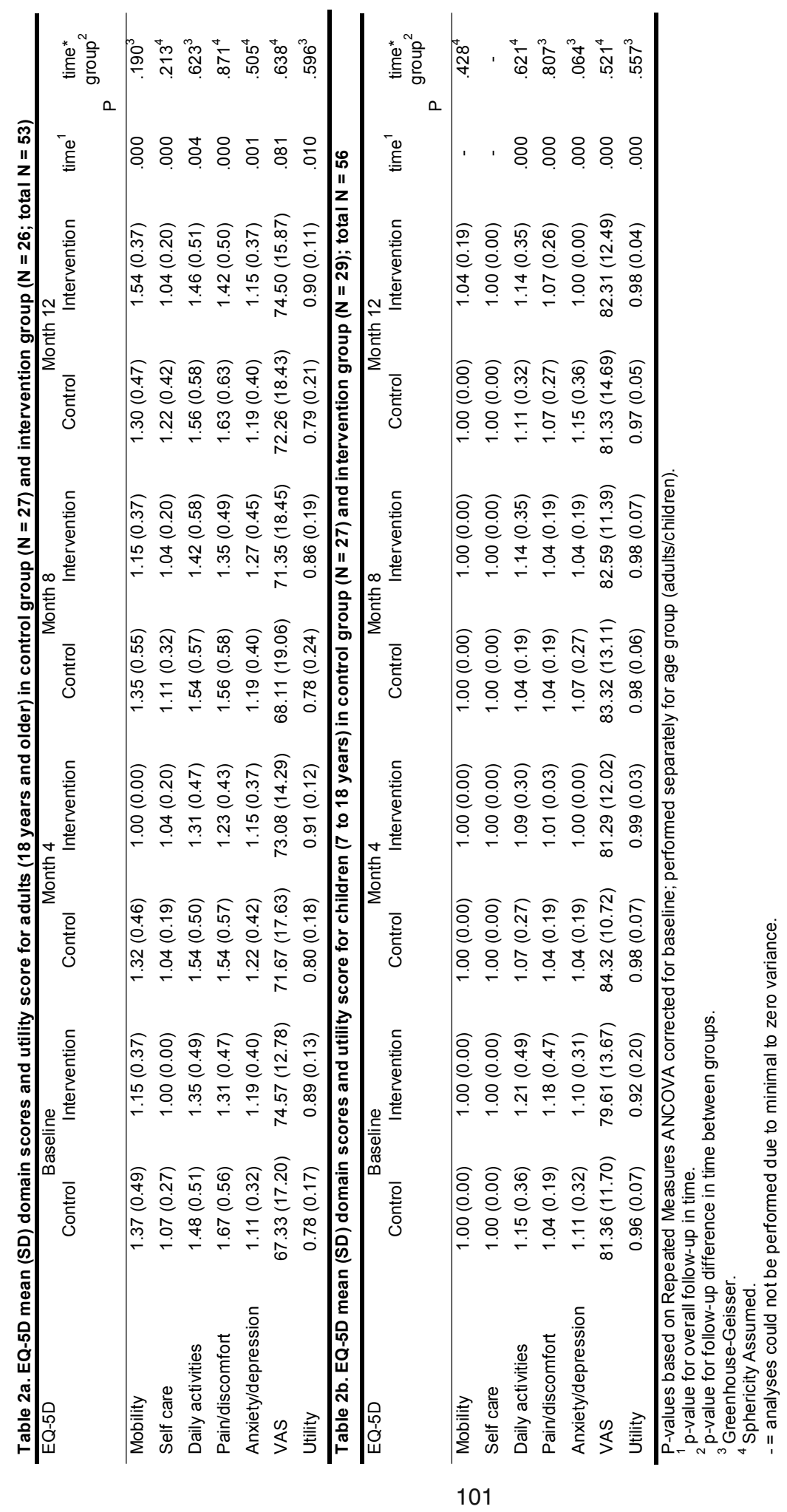




\section{Chapter 6}

results of the SF-6D among the adult participants showed the same pattern as the scores on the EQ-5D. Differences between the groups in time were not statistically significant. See table 3.

Table 3. SF36 mean (SD) domain scores and SF-6D mean (SD) utility score for adult (18 years and older) study participants in the control group $(\mathrm{N}=27)$ and intervention group $(\mathrm{N}=26)$

\begin{tabular}{|c|c|c|c|c|c|c|c|c|c|c|}
\hline \multirow[t]{2}{*}{ SF36 } & \multicolumn{2}{|c|}{ Baseline } & \multicolumn{2}{|c|}{ Month 4} & \multicolumn{2}{|c|}{ Month 8} & \multicolumn{2}{|c|}{ Month 12} & \multirow{2}{*}{ time $^{1}$} & \multirow{2}{*}{$\begin{array}{l}\text { time }^{*} \\
\text { group }\end{array}$} \\
\hline & C & 1 & C & 1 & C & 1 & C & 1 & & \\
\hline $\begin{array}{l}\text { Physical } \\
\text { functioning }\end{array}$ & $\begin{array}{l}68.06 \\
(24.3)\end{array}$ & $\begin{array}{l}72.42 \\
(20.9)\end{array}$ & $\begin{array}{l}67.05 \\
(25.7)\end{array}$ & $\begin{array}{l}75.62 \\
(21.5)\end{array}$ & $\begin{array}{l}66.02 \\
(26.9)\end{array}$ & $\begin{array}{l}71.20 \\
(23.9)\end{array}$ & $\begin{array}{l}68.79 \\
(25.7)\end{array}$ & $\begin{array}{l}71.58 \\
(22.6)\end{array}$ & .088 & $.412^{4}$ \\
\hline $\begin{array}{l}\text { Social } \\
\text { functioning }\end{array}$ & $\begin{array}{l}66.20 \\
(26.4)\end{array}$ & $\begin{array}{l}75.96 \\
(26.4)\end{array}$ & $\begin{array}{l}71.99 \\
(26.3)\end{array}$ & $\begin{array}{c}76.92 \\
(20.52)\end{array}$ & $\begin{array}{l}71.99 \\
(27.0)\end{array}$ & $\begin{array}{l}74.52 \\
(25.4)\end{array}$ & $\begin{array}{l}72.22 \\
(25.6)\end{array}$ & $\begin{array}{l}78.37 \\
(22.0)\end{array}$ & .000 & $.743^{4}$ \\
\hline $\begin{array}{l}\text { Role } \\
\text { physical }\end{array}$ & $\begin{array}{l}42.59 \\
(46.4)\end{array}$ & $\begin{array}{l}49.85 \\
(44.2)\end{array}$ & $\begin{array}{l}48.61 \\
(43.9)\end{array}$ & $\begin{array}{l}56.73 \\
(43.9)\end{array}$ & $\begin{array}{l}54.17 \\
(47.2)\end{array}$ & $\begin{array}{l}47.12 \\
(45.5)\end{array}$ & $\begin{array}{l}57.41 \\
(45.9)\end{array}$ & $\begin{array}{l}53.53 \\
(45.5)\end{array}$ & .001 & $.327^{4}$ \\
\hline $\begin{array}{l}\text { Role } \\
\text { emotional }\end{array}$ & $\begin{array}{l}65.50 \\
(41.8)\end{array}$ & $\begin{array}{l}71.80 \\
(41.8)\end{array}$ & $\begin{array}{l}65.43 \\
(45.0)\end{array}$ & $\begin{array}{l}71.80 \\
(41.8)\end{array}$ & $\begin{array}{l}76.54 \\
(41.2)\end{array}$ & $\begin{array}{l}67.95 \\
(46.7)\end{array}$ & $\begin{array}{l}77.78 \\
(40.3)\end{array}$ & $\begin{array}{l}78.21 \\
(38.8)\end{array}$ & .000 & $.438^{4}$ \\
\hline $\begin{array}{l}\text { Mental } \\
\text { health }\end{array}$ & $\begin{array}{l}71.74 \\
(17.4)\end{array}$ & $\begin{array}{l}75.29 \\
(18.7)\end{array}$ & $\begin{array}{l}74.30 \\
(17.7)\end{array}$ & $\begin{array}{l}71.39 \\
(21.5)\end{array}$ & $\begin{array}{l}71.56 \\
(18.9)\end{array}$ & $\begin{array}{l}73.69 \\
(22.2)\end{array}$ & $\begin{array}{l}76.59 \\
(17.6)\end{array}$ & $\begin{array}{l}77.23 \\
(18.4)\end{array}$ & .004 & $.335^{3}$ \\
\hline Vitality & $\begin{array}{l}52.96 \\
(19.6)\end{array}$ & $\begin{array}{l}58.68 \\
(20.9)\end{array}$ & $\begin{array}{l}53.92 \\
(17.7)\end{array}$ & $\begin{array}{l}59.23 \\
(22.4)\end{array}$ & $\begin{array}{l}53.61 \\
(21.3)\end{array}$ & $\begin{array}{l}55.77 \\
(21.7)\end{array}$ & $\begin{array}{l}59.44 \\
(22.9)\end{array}$ & $\begin{array}{l}60.77 \\
(23.6)\end{array}$ & .074 & $.574^{3}$ \\
\hline Bodily Pain & $\begin{array}{l}64.96 \\
(25.6)\end{array}$ & $\begin{array}{l}75.15 \\
(24.4)\end{array}$ & $\begin{array}{l}66.37 \\
(25.2)\end{array}$ & $\begin{array}{l}84.35 \\
(18.9)\end{array}$ & $\begin{array}{c}65.26 \\
(24.21)\end{array}$ & $\begin{array}{l}72.00 \\
(24.9)\end{array}$ & $\begin{array}{l}67.59 \\
(23.3)\end{array}$ & $\begin{array}{l}74.92 \\
(21.7)\end{array}$ & .000 & $.064^{4}$ \\
\hline $\begin{array}{l}\text { General } \\
\text { Health }\end{array}$ & $\begin{array}{l}48.55 \\
(21.8)\end{array}$ & $\begin{array}{l}47.31 \\
(22.0)\end{array}$ & $\begin{array}{c}48.83 \\
(22.40)\end{array}$ & $\begin{array}{l}51.72 \\
(21.7)\end{array}$ & $\begin{array}{l}48.65 \\
(23.9)\end{array}$ & $\begin{array}{l}50.89 \\
(24.2)\end{array}$ & $\begin{array}{l}52.05 \\
(24.2)\end{array}$ & $\begin{array}{l}50.05 \\
(21.3)\end{array}$ & .011 & $.459^{3}$ \\
\hline $\begin{array}{l}\text { SF-6D } \\
\text { utility }\end{array}$ & $\begin{array}{c}0.69 \\
(0.14)\end{array}$ & $\begin{array}{c}0.75 \\
(0.13)\end{array}$ & $\begin{array}{c}0.72 \\
(0.14)\end{array}$ & $\begin{array}{c}0.75 \\
(0.13)\end{array}$ & $\begin{array}{c}0.71 \\
(0.16)\end{array}$ & $\begin{array}{c}0.74 \\
(0.15)\end{array}$ & $\begin{array}{c}0.74 \\
(0.14)\end{array}$ & $\begin{array}{c}0.75 \\
(0.14)\end{array}$ & .012 & $.301^{3}$ \\
\hline
\end{tabular}

$\mathrm{C}=$ control group; $\mathrm{I}=$ intervention group.

Domain scores range $0-100$.

P-values based on Repeated Measures ANCOVA corrected for baseline.

${ }^{1} \mathrm{p}$-value for overall follow-up in time.

$2 \mathrm{p}$-value for follow-up difference in time between groups.

${ }^{3}$ Greenhouse-Geisser

${ }^{4}$ Sphericity Assumed.

The children in the control group experienced more problems with regard to anxiety/depression on the EQ-5D at month 12 compared to the intervention group. The mean utility scores were slightly higher in the control group at baseline and all equalised during follow-up measurements. In both groups the domain scores statistically improved in time, but no statistical significant differences between the groups were observed. 
Costs

Table 4 presents the mean (SD) costs for adults (18 years and older) and children ( 7 to 18 years) separate. The mean one year intervention costs amounted to $€ 530$ (SD $€ 57$ ) per adult and $€ 537$ (SD $€ 54$ ) per child. The largest part of the intervention costs was associated with the fixed costs ( $€ 473)$, and on average only a small proportion of the intervention costs was variable $(€ 37, \mathrm{SD} € 12$ ). Overall, the mean health care costs per patient were higher in the intervention group $(€ 2,228, S D € 1,582$ adults; $€ 1,193, S D €$ 582 children) than in the control group ( $€ 1,720, S D € 1,742$ adults; $€ 588$, SD $€ 850$; children). The cost difference between the groups was mainly caused by the intervention costs. Apart of the intervention costs, other costs were also slightly higher in the intervention group. Besides the intervention costs, in both groups medication costs and costs of professional home care accounted for a large proportion of the health care costs.

With regard to the adults the costs of informal care were on average twice as high in the intervention group ( $€ 127, S D € 323$ ), as in the control group ( $€ 62$, SD $€ 214$ ). As a result the difference in patient and family costs between the groups is somewhat larger than the difference in health care costs. The costs due to loss of productivity at paid labour are on average five times higher among the patients in the intervention group ( $€$ 487 , SD $€ 1,394$ ), than among the patients in the control group (€99, SD $€ 281$ ). As a result, the total costs for adults and children are higher in the intervention group $(€ 2,973$, SD $€ 2,650$ adults; $€ 1,206, S D € 601$ children), than in the control group ( $€ 1,948, S D €$ 1,777 adults; $€ 597$, SD $€ 863$ children).

Incremental cost-effectiveness

The results of the incremental analysis are listed in table 5. Among the adults the health care costs were on average $€ 508$ higher in the intervention group. Based on the bootstrap simulation, the $95 \%$ confidence interval $(\mathrm{Cl})$ of the difference in health care costs ranged from $-€ 114$ to $€ 1,118$. The difference in societal costs was larger ( $€ 1,026$; 2.5th percentile $€ 231$; 97 th percentile $€ 1,889$ ), due to the higher productivity losses in the intervention group. Based on EQ-5D utility, after adjustment for baseline differences by multiple regression, on average 0.03 QALY (2.5th percentile 0.00; 97th percentile 0.07 bootstrap analysis) was gained from the intervention. The mean incremental costeffectiveness ratio was $€ 15,366$ /QALY gained from the health care perspective and $€$ 31,035 /QALY gained from the societal perspective. The cost-effectiveness acceptability curve shows that the probability of cost-effectiveness was $85 \%$ at a ceiling ratio of $€$ 


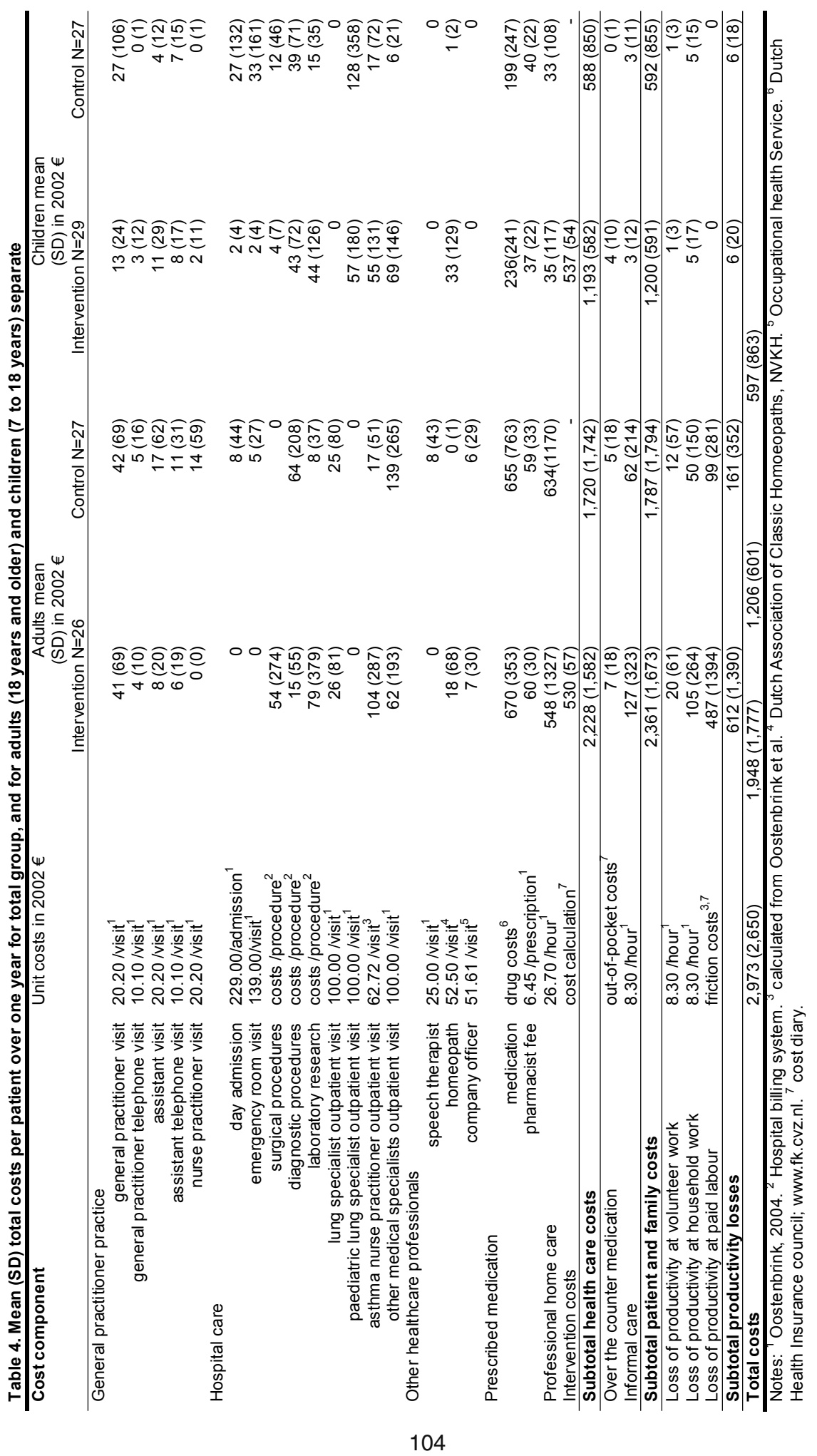


80,000 /QALY gained and $59 \%$ at a ceiling ratio of $€ 40,000$ /QALY gained from the societal perspective. Based on the SF-6D utility the mean incremental cost-effectiveness ratio were inferior from both the health care as the societal perspective.

Among the children health care costs were $€ 605$ higher in the intervention group (2.5th percentile $€ 319$; 97 th percentile $€ 862$ ). The difference in societal costs was about the same ( $€ 609$; 2.5th percentile $€ 312$; 97th percentile $€ 864$ ). The utility adjusted for baseline differences by multiple regression on average 0.01 QALY (2.5th percentile 0.00 ; 97th percentile 0.02 ) was gained from the intervention. From the health care perspective the mean ICER was $€ 58,726 /$ QALY and from the societal perspective $€ 59,071$ /QALY gained. The cost-effectiveness acceptability curve show that the probability of acceptance is $68 \%$ at a ceiling ratio of $€ 80,000 /$ QALY gained and $22 \%$ at a ceiling ratio of $€ 40,000$ /QALY gained from the societal perspective.

\section{Sensitivity analysis}

Table 5 presents a sensitivity analysis in which the monitor costs were set to zero and a sensitivity analysis in which the costs resulting from school absenteeism were included.

After leaving out the monitor costs, among the adults the incremental health care costs changed from $€ 508$ to $€ 58$. The incremental societal costs decreased from $€ 1,026$ to $€$ 576. From the health care prospective the mean incremental cost-effectiveness ratio changed from $€ 15,366 / Q A L Y$ gained to $€ 1,759$ /QALY gained and from the societal perspective from $€ 31,035 /$ QALY gained to $€ 17,427$ /QALY gained. The probability of cost-effectiveness at a ceiling ratio of $€ 80,000$ /QALY gained, changed from $85 \%$ to $90 \%$ from the societal perspective. The incremental cost-effectiveness ratios based on the SF-6D utility remained inferior. Among the children the incremental health care costs changed from $€ 605$ to $€ 156$ and the societal costs from $€ 609$ to $€ 159$. From the societal perspective the cost-effectiveness ratio changed from $€ 59,071 / Q A L Y$ gained to $€ 15,438$ /QALY gained. The probability that that the intervention was cost-effective changed from $68 \%$ to $93 \%$ at a ceiling ratio of $€ 80,000 /$ QALY gained. The figures 1,2 and 3 present cost-effectiveness curves and cost-effectiveness planes of the base case and the sensitivity analyses, in which the monitor costs were subtracted.

In the control group 7 children had on average 22 hours of school absenteeism and in the intervention group 6 children had on average 5 hours of school absenteeism. As a result the costs of school absenteeism were $€ 86$ (SD $€ 272$ ) in the control group and $€$ 20 (SD $€ 85$ ) in the intervention group. Taking these costs into account the incremental societal costs changed from $€ 609$ to $€ 542$ (2.5th percentile $€ 196$; 97 th percentile $€$ 849). The cost-effectiveness ratio changed from $€ 59,071 / Q A L Y$ gained to $€ 52,618$ 
/QALY gained. At a ceiling ratio of $€ 80,000$ /QALY gained, the probability of costeffectiveness changed from $68 \%$ to $73 \%$ from the societal perspective.

Table 5. Incremental costs, effects, and cost-effectiveness analysis

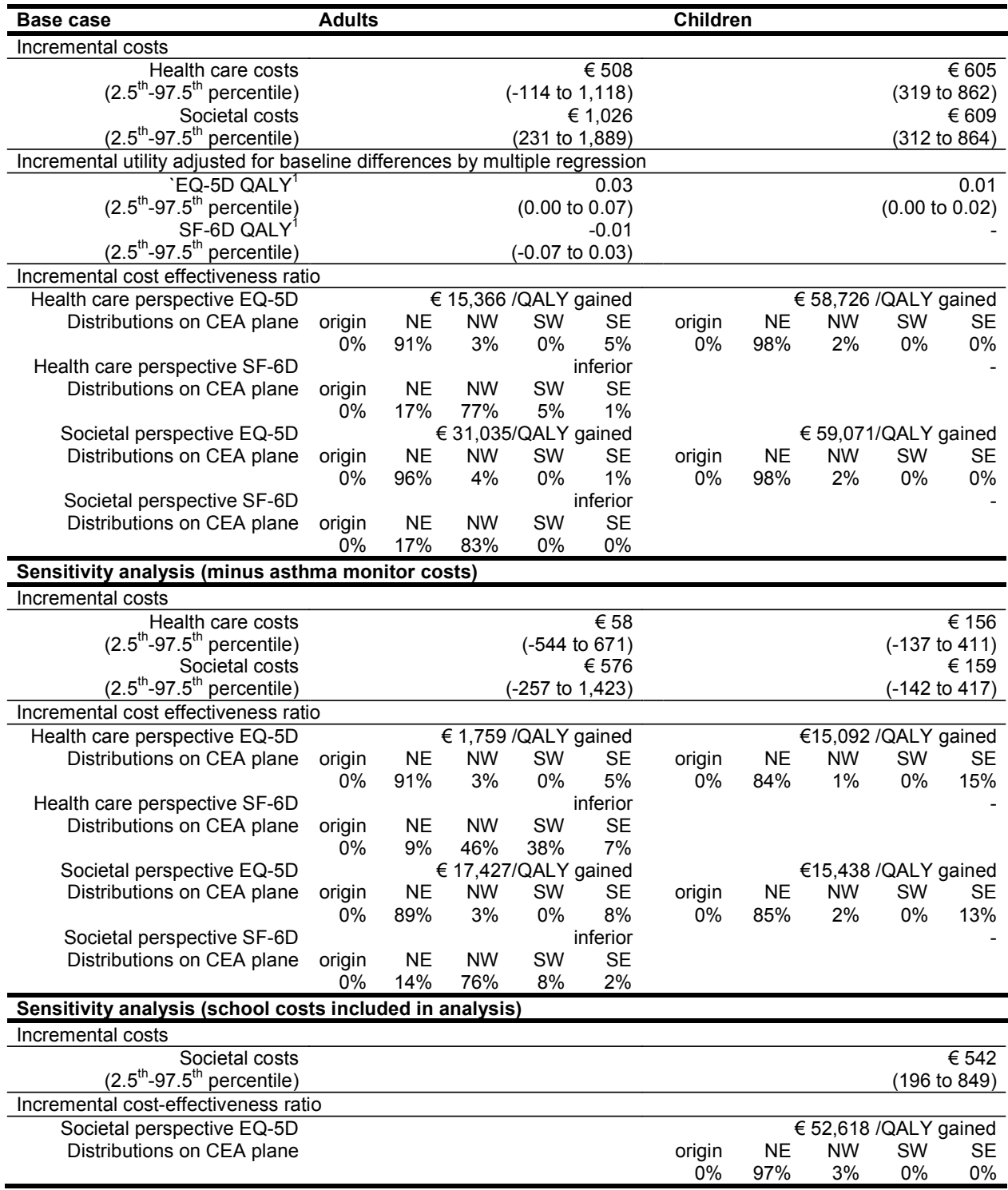

Costs year 2002 in euro.

ICER = Incremental Cost-effectiveness Ratio

Percentiles based on bootstrap procedure with 5000 replicates.

${ }^{1}$ Utility scores at follow-up measurements are adjusted for between group baseline differences using the multiple regression method. 
Figure 1a and 1b. Cost-effectiveness plane and cost-effectiveness acceptability curves (base case and minus the asthma monitor costs) from a societal perspective with EQ-5D utility for adults
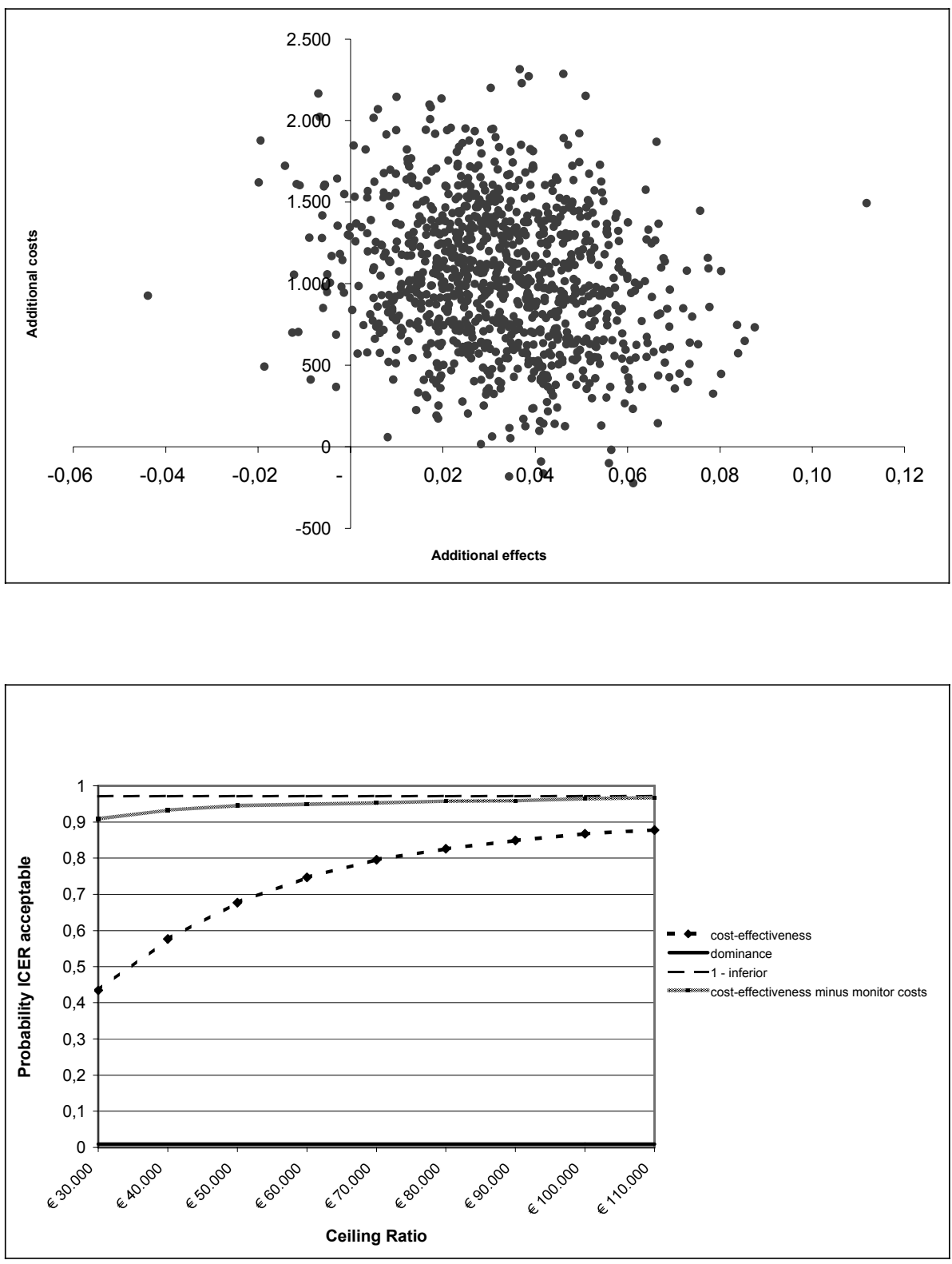
Chapter 6

Figure $2 \mathrm{a}$ and $2 \mathrm{~b}$. Cost-effectiveness plane and cost-effectiveness acceptability curves (base case and minus the asthma monitor costs) from a societal perspective with EQ-5D utility for children
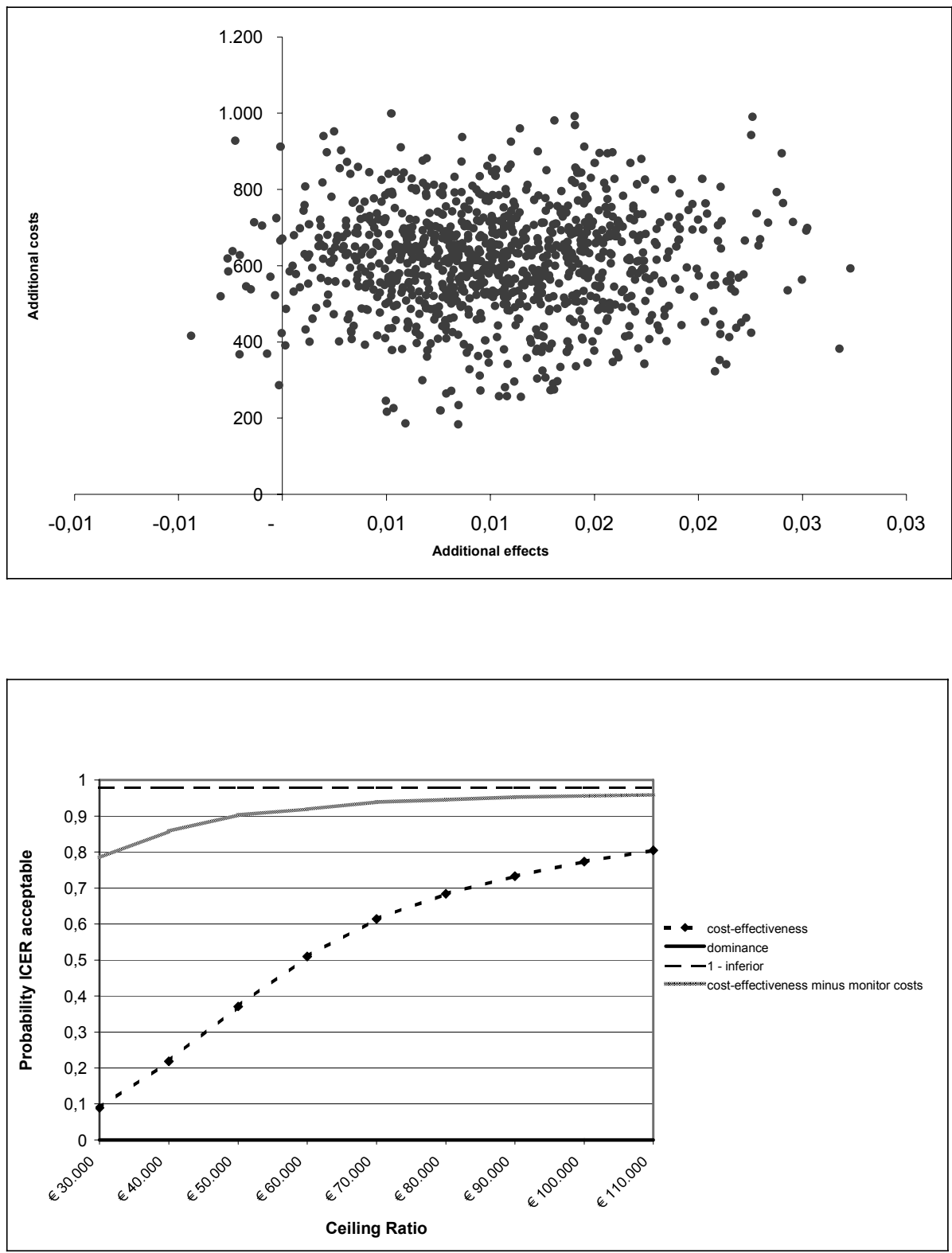
Figure $3 \mathrm{a}$ and $3 \mathrm{~b}$. Cost-effectiveness plane and cost-effectiveness acceptability curves (base case and minus the asthma monitor costs) from a societal perspective with SF-6D utility for adults
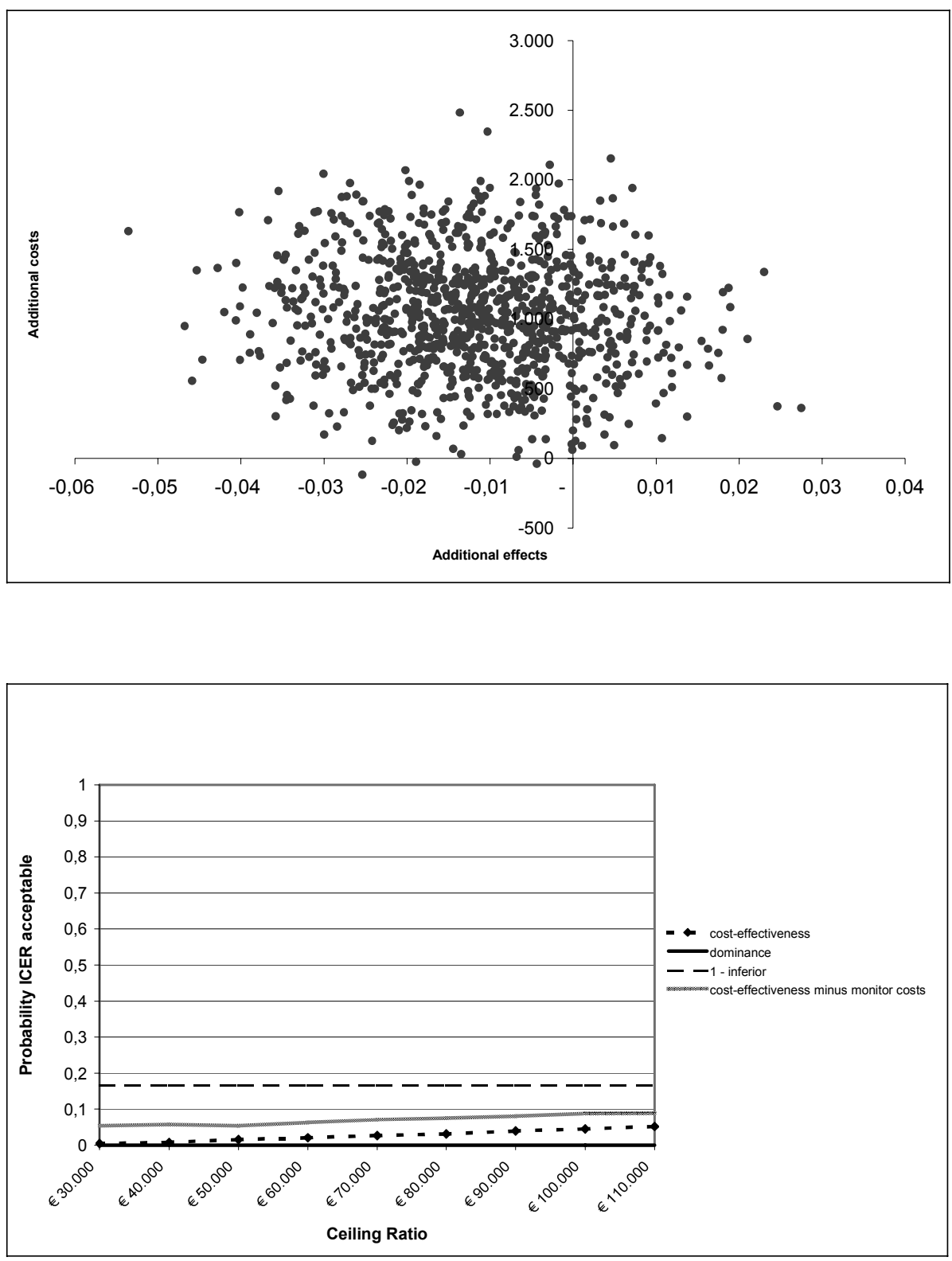


\section{Chapter 6}

\section{Discussion}

This study compared the cost-effectiveness of a nurse-led telemonitoring programme in asthmatic outpatients to regular care. Patients were asked to transfer their monitor data at least twice daily and by judging the received data and following a stepwise intervention protocol the nurse practitioner was able to act as the main care provider in the intervention group. In both groups the EQ-5D and the SF-6D were used to obtain estimates of health state utilities. One year, health care costs, patient and family costs, productivity losses were calculated. The mean incremental costs were weighted against the mean incremental effects in terms of QALY.

No significant differences were found between the groups with regard to generic quality of life. Overall, the mean health care costs per patient were higher in the intervention group than in the control group. The intervention costs mainly caused the cost difference between the groups, but not totally. The intervention costs the society $€ 31,035$ /QALY gained among adults and among children $€ 59,071$ /QALY gained. From the societal perspective the probability of cost-effectiveness is $85 \%$ at a ceiling ratio of $€ 80,000$ IQALY gained among the adults and $68 \%$ among the children. When the QALY was based on the SF-6D utility the mean incremental cost-effectiveness ratios were inferior from both the health care as the societal perspective. In conclusion the intervention appears to be more cost-effective among adults.

If the monitor costs are excluded the incremental costs decrease strongly among both the adults and the children. The decrease of monitor costs results in a far more costeffective intervention. Given the fast developments in information communication technology, this decrease in price is expected to take place in the future. If the costs with regard to the children's absence from school are included, the cost-effectiveness ratio only decreases about $€ 6,000$, and the probability of cost-effectiveness is still moderate. With regard to the higher costs due to productivity losses in the intervention group caution should be taken, as in the intervention group a greater proportion of patients were paid employers.

Considering the results of this study the intervention is certainly not cost saving, and especially among children, of limited cost-effectiveness. This result is in contrast with the results of a recent review, in which was found that only in three out of twenty-one economic evaluations self-management programmes based on peak flow monitoring, the total costs were higher in the intervention group (25).

As described in a review of Gibson et al. (2003), education in asthma-management which involves self-monitoring, regular medical review, and a written action plan appear to improve health outcomes the most. In this study no written action plans were used 
which may have reduced the effectiveness. Therefore, it might be recommended to entail this aspect in future asthma self-management programmes. Furthermore, it is necessary to be cautious in generalising these findings, as this is an one centre study in a Dutch outpatient setting. Also, these results should not be generalised to asthmatics with more severe symptoms or the results of economic evaluations with a longer time horizon than one year. Another study limitation is the use of interpolated diary data which can lead to an underestimation or overestimation of the actual resource use during the follow-up year. However, administrating diaries daily during one year is very aggravating and may negatively influence recruitment and drop out. In general, diaries are an accepted way to measure resource use and productivity losses $(5 ; 18)$. Moreover, any bias is likely to have occurred in both groups. Furthermore, the questionnaire and diary compliance were very high. For these reasons a simple strategy to impute missing data seems justified.

In general the study population had mild to moderate asthma and the patients did not report many asthma symptoms. This is in accordance with the observed high scores in the self-reported quality of life. Nevertheless these patients were diagnosed with asthma and received regular outpatient care for that reason. Home monitoring has been suggested to be the most efficient for asthmatics who are poor perceivers of asthma severity (Fishwick 1996). On the other hand, asthma patients with moderate till severe asthma may have more benefit of the intervention, since there is more room for improvement. This is confirmed in the review of Liljas et al. (1994), in which was stated that asthma self-management seems to be even more cost-effective in patients with severe asthma. The low variation in the generic quality of life scores may also imply that the questionnaires were not sensitive enough for this particular group, especially among children. Though the patients only reported mild asthma symptoms, the health care costs in the study population were relatively high, mainly caused by the intervention and medication costs. This stresses the importance of health services research in asthma self-management programmes, in order to maximise the effects and minimise the costs of medical therapy.

Another reason why the intervention was of limited cost-effectiveness could be that the intervention was initiated separately instead of integrated into the total process of care. That way the intervention may only partially contribute to the improvement of the total care process, both in terms of health outcomes and costs. This underlines the importance of integrated care in telemonitoring programmes. 


\section{Chapter 6}

\section{Conclusions}

In conclusion, if the outcome is measured by generic quality of life the nurse-led telemonitoring programme is of limited cost-effectiveness in the study population. From the societal perspective the probability of the programme being cost-effective compared to regular care was $85 \%$ at a ceiling ratio of $€ 80,000$ /QALY gained among the adults and $68 \%$ among the children. However, a decrease in the price of the asthma monitor will substantially increase the probability of the programme to be cost-effective. 


\section{References}

1. World Health Organization (WHO). Bronchial asthma: The scale of the problem. htpp://www.who.int/mediacentre/factsheets/fs206/en/; 2000.

2. Masoli M, Fabian D, Holt S, Beasley R: Global Initiative for Asthma (GINA): global burden of asthma. http://www.ginasthma.com/Reportltem.asp?!1=2\&l2=2\&intld=94; 2004.

3. Hoogendoorn M, Feenstra TL, Rutten-van Mölken MPMH: Projections of future resource use and the costs of asthma and COPD in the Netherlands (in Dutch). Ned Tijdschr Geneeskd 2006, 150:1243-50.

4. Cisternas MG, Blanc PD, Yen IH, Katz PP, Earnest G, Eisner MD, Shiboski S, Yelin EH: A comprehensive study of the direct and indirect costs of adult asthma. J Allergy Clin Immunol 2003, 111:1212-1218.

5. Drummond MF, Sculpher MJ, Torrance GW, O'Brien BJ, Stoddart GL: Methods for the economic evaluation of health care programmes (Third Edition). Oxford: Oxford University Press 2005.

6. Meystre S: The current state of telemonitoring: a comment on the literature. Telemed J E Health 2005, 11:6369.

7. Horrocks S, Anderson E, Salisbury C: Systematic review of whether nurse practitioners working in primary care can provide equivalent care to doctors. BMJ 2002, 324:819-823.

8. Willems DC, Joore MA, Hendriks JJ, Wouters EF, Severens JL: Cost-effectiveness of self-management in asthma: A systematic review of peak flow monitoring interventions. Int J Technol Assess Health Care 2006, 22:436-442.

9. Juniper EF, Guyatt GH, Ferrie PJ, Griffith LE. Measuring quality of life in asthma. Am Rev Respir Dis 1993, 147:832-838.

10. Juniper EF, Guyatt GH, Feeny DH, Ferrie PJ, Griffith LE, Townsend M: Measuring quality of life in children with asthma. Qual Life Res 1996, 5:35-46.

11. Juniper EF, Guyatt GH, Feeny DH, Ferrie PJ, Griffith LE, Townsend M: Measuring quality of life in the parents of children with asthma. Qual Life Res 1996, 5:27-34.

12. Willems DCM, Joore MA, Hendriks JJE, van Duurling RAH, Wouters EFM, Severens JL: The process evaluation of a nurse-led telemonitoring programme for asthmatics. J Telemed Telecare, in press.

13. Brooks R: EuroQol: the current state of play. Health Policy 1996, 37:53-72.

14. Dolan P: Modeling valuations for EuroQol health states. Med Care 1997, 35:1095-1108.

15. Stolk EA, Busschbach JJ, Vogels T: Performance of the EuroQol in children with imperforate anus. Qual Life Res 2000, 9:29-38.

16. Brazier J, Usherwood T, Harper R, Thomas K: Deriving a preference-based single index from the UK SF-36 Health Survey. J Clin Epidemiol 1998, 51:1115-28.

17. Brazier J, Roberts J, Deverill M: The estimation of a preference-based measure of health from the SF-36. J Health Econ 2002; 21:271-292.

18. Goossens MEJB, Rutten-van Mölken MPMH, Vlaeyen JWS, van der Linden SMJP: The cost diary: a method to measure direct and indirect costs in cost effectiveness research. J Clin Epidemiol 2000, 53:688-695.

19. Oostenbrink JB, Boumans CAM, Koopmanschap MA, Rutten FFH: Manual for Cost Research. (In Dutch). Amstelveen: Health Insurance Board; 2004.

20. Koopmanschap MA, Rutten FFH, van Ineveld BM, van Roijen L: The friction cost method for measuring indirect costs of disease. J Health Econ 1995, 14:171-189.

21. Ritico JA, de Heij LJM, van Luij JCF, Wolff I: Report. Guidelines for Pharmaco-economic Research (In Dutch). Amstelveen: Health Insurance Board; 1999.

22. Ministry of Education, Culture and Science (in Dutch). http://www.minocw.nl/start

23. Briggs AH, Wonderling DE, Mooney CZ: Pulling cost-effectiveness analysis up by its bootstraps: a nonparametric approach to confidence interval estimation. J Health Econ 1997, 6:327-340.

24. Ministry of Health, Welfare and sports (in Dutch). http://www.rvz.net/data/download/advies_Zinnige_zorg.pdf 


\section{Chapter 6}

25. Willems DC, Joore MA, Hendriks JJ, Wouters EF, Severens JL: Cost-effectiveness of self-management in asthma: A systematic review of peak flow monitoring interventions. Int J Technol Assess Health Care 2006, 22:436-442.

26. Gibson PG, Powell H, Coughlan J, Wilson AJ, Abramson M, Haywood P, Bauman A, Hensley MJ, Walters EH: Self-management education and regular practitioner review for adults with asthma (Cochrane Review) Cochrane Database Syst Rev 2003, Issue 1:CD001117.

27. Fishwick D, Beasley R: Use of peak flow-based self-management plans by adult asthmatic patients. Eur Respir J 1996, 9:861-865.

28. Liljas B, Lahdensuo A: Is asthma self-management cost-effective? Patient Educ Couns 1997, 32 Suppl 1:97104. 
General discussion

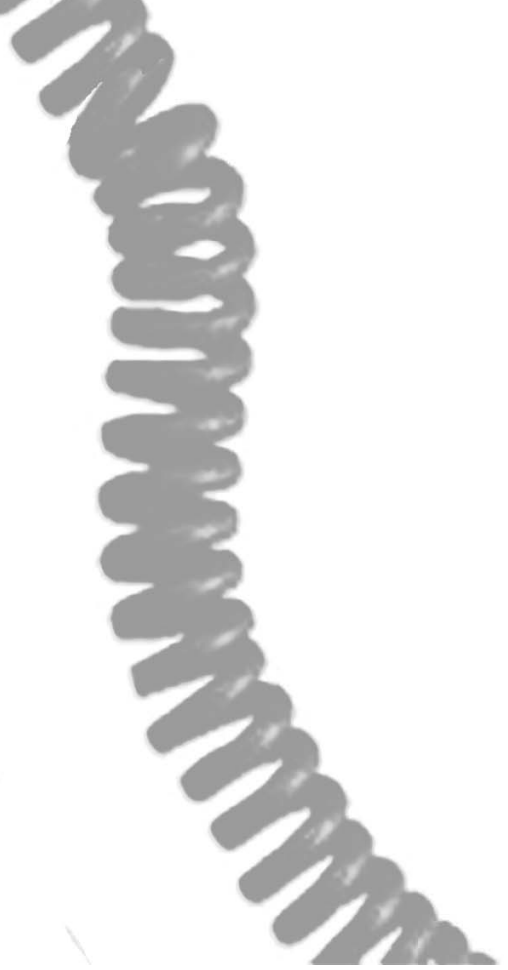




\section{Chapter 7}

\section{General discussion}

This thesis concerns the process, health outcomes and cost-effectiveness of a nurse-led telemonitoring programme in asthmatic outpatients. The nurse-led telemonitoring programme was developed for asthmatic outpatients, from the age of seven years, and the study follow-up was one year. The programme was compared to regular care and both the costs and outcomes were examined in a cost-effectiveness analysis. In addition, the use of a multi-attribute utility instrument, the so called EQ-5D, was explored in children with chronic conditions.

To specify the objective of this thesis five research questions were formulated which are presented in the general introduction.

In this chapter, a reflection is given on the main findings, the methodological findings, and conclusions of all the chapters of this thesis. Furthermore, the generalisation of the study results and the limitations of the study will be discussed. Policy recommendations will be given as well as suggestions for future research.

\section{Main findings}

\section{Cost-effectiveness of peak flow monitoring interventions in asthma}

In chapter 2 an overview was given of 21 economic evaluations of peak flow monitoring interventions in asthma in a systematic review. The methodological and economic quality of these economic evaluations were assessed with the use of a checklist. The mean methodological quality was 4.6 (maximum 8 ), and the mean economic quality was 12.0 (maximum 15). The economic evaluations appeared to differ extremely regarding interventions, costs and outcomes. Furthermore, specific details were often not presented. In addition, asthma quality of life outcomes were measured in only 10 studies, and generic quality of life outcomes in only six studies. Merely one study expressed the health outcomes as utilities, and calculated QALYs. In 13 of the 17 full economic evaluations at least one of the reported health outcomes showed a statistically significant improvement after the intervention. These related especially to clinical symptoms. Of the 17 full economic evaluations the intervention (or more intensive intervention) was dominant in no less than 14 studies, four of which described the difference as statistically significant. 


\section{Process evaluation of the telemonitoring programme}

In chapter 3 a process evaluation of a nurse-led telemonitoring programme in asthma was performed. In this programme a nurse practitioner is the main care provider and patients are requested to use an electronic peak flow monitor at home twice daily. The indicators for the process evaluation were the feasibility of the intervention, the consistency of the PEF tests, compliance with both the study and intervention protocols, and patient satisfaction with the programme. The willingness to participate in the study was $40 \%$. Furthermore, the drop out rate during the follow-up was low. The validity of the PEF manoeuvres was on average good and the compliance with the study protocol was high. Although the maximum burden of measurements per day was reached (twice daily), the patient satisfaction was high. The results indicate that, if a self-management programme is introduced into a motivated group of mild to moderate asthmatics, the programme is feasible, reliable, and satisfying.

\section{Effects and their relationships in the telemonitoring programme}

In chapter 4 the effects on, and the relationship between, asthma symptoms, asthmaspecific quality of life, and medical consumption of the nurse-led telemonitoring programme was compared to regular outpatient care. Overall, the intervention in this study did not significantly decrease asthma symptoms or medical consumption, or improve asthma-specific quality of life. The internal correlations were on average moderate to high in the expected direction and all these internal correlations were statistically significant. The lung function value PEF had a very low correlation with the self-reported use of corticosteroids and the PEF and FEV1 had a low correlation with the Activity Limitation domain of the (P)AQLQ, as expected. Very low, non-significant correlations were present between the symptoms and the other outcome parameters. With regard to medication, very low and non-significant correlations were found between the domains of asthma-specific quality of life and the self-reported use of corticosteroids, but remarkably low statistically significant correlations were found between all three domains of asthma-specific quality of life and self-reported use of beta-2 agonists. This suggests that the use of beta- 2 agonists relates stronger to asthma-specific quality of life than to self-reported symptoms.

\section{The use of the EQ-5D child version}

In chapter 5 the use of the parent form and child form of the EQ-5D in children with chronic conditions was explored. For this purpose the feasibility, construct validity, discriminant ability, and test-retest correlations were investigated. The children were 7- 


\section{Chapter 7}

18 years old with one of the following four conditions: asthma, rheumatic disorders, diabetes, or speech/language/hearing disorder. In conclusion, for children with a cognitive impairment and a variable health status the EQ-5D seems to be less suitable. However, the EQ-5D could be easily administrated among children and parents of children with chronic conditions. The EQ-5D seems a valid questionnaire for children with a chronic physical condition and reliable for children with a stable health status.

\section{The cost-effectiveness of the telemonitoring programme}

In chapter 6 the cost-effectiveness of the nurse-led telemonitoring programme was determined and compared to regular outpatient care. Measuring the outcomes by means of a generic quality of life questionnaire resulted in limited cost-effectiveness of the programme in the study population. Overall, the mean health care costs per patient were higher in the intervention group than in the control group. The intervention costs mainly caused the cost difference between the groups. From the societal perspective the probability of the programme being cost-effective compared to regular care was $85 \%$ at a ceiling ratio of $€ 80,000 /$ QALY gained among the adults and $68 \%$ among the children. The sensitivity analyses showed that if these costs were excluded, the incremental costs decreased strongly among both the adults and the children. Decreases in the price of the asthma monitor will substantially increase the probability of cost-effectiveness of the programme.

\section{Methodological findings}

\section{Cost-effectiveness of peak flow monitoring interventions in asthma}

The methodological quality of most of the economic evaluations of self-management based on peak flow monitoring interventions in asthma was on average low. These results show that there is a need for guidelines to increase the quality and comparability of cost-effectiveness evaluations relating to asthma. Only then will it be possible to conclude whether interventions for asthmatics, such as self-management based on peak flow monitoring interventions, are cost-effective.

\section{Process evaluation of the telemonitoring programme}

Evaluation of processes is very important to obtain quality improvement. Up to now there is no uniform framework to investigate the process of new interventions in health care. The development of means to implement effective asthma telemonitoring programmes also includes the use of appropriate tools to measure success. The results of process evaluations in telemedicine programmes should be shared whether these results are 
positive or negative. Such process evaluations can provide cohesive information on the application of a programme within the target population. They help explain the success or lack of effect of a programme and can thereby enable others to replicate the programme and generate improvements. In this way the cycle of continuous quality improvement can begin.

\section{Effects and their relationships in the telemonitoring programme}

The study participation rate was $40 \%$ and 'having no time' was the main reason for not participating in this programme. This indicates that it is very important that future telemonitoring programmes should be as little time-consuming as possible. Also, it can be concluded that there are technical and logistical barriers to overcome regarding nurse-led self-management interventions. The results showed that a self-management programme on its own is not a guarantee of success. The findings of this study emphasise that a telemonitoring programme is not feasible for all asthmatics. The programme should be offered to patients who are motivated since patients themselves are the decision makers in their self-management. The cost-effectiveness of a nurse-led telemonitoring programme may improve when the programme is introduced to a motivated group of not well controlled patients with mild to moderate asthma.

\section{The use of the EQ-5D child version}

It appears that multi-attribute utility instruments developed for adult patients may not be suitable for children without adjustments. For these reasons caution should be taken when using EQ-5D derived QALY scores in child populations. The EQ-5D child version appears to be an unreliable estimate of the effectiveness in terms of utility gain in child asthma management. In the future it is recommended that research will take place, to investigate to what extent preferences change, if age is taken into account. Depending on the outcomes, eventually this could lead to the development of generic preference based utility functions for children. Furthermore, this instrument should account for the adaptation of children to their situation and the children's view about their condition and may have additional value to measure quality of life in children.

\section{The cost-effectiveness of the telemonitoring programme}

The study population had mild to moderate asthma and their quality of life scores were on average very high and showed low variation. The limited cost-effectiveness could be related to the questionnaires used which probably were not sensitive enough for this particular group, especially among children. Another reason why the intervention was of 


\section{Chapter 7}

limited cost-effectiveness could be that the intervention was initiated separately instead of integrated into the total process of care.

Furthermore, it is necessary to be cautious in generalising these findings, as this is a one centre study in a Dutch outpatient setting. In future research it is important that telemonitoring programmes are integrated in the total care process. Asthma patients with moderate to severe asthma may benefit more from the intervention, since there is more room for improvement. Furthermore, the use of written action plans may decrease the effectiveness.

\section{Limitations}

During the years the study was executed, the rationale behind asthma management has changed. Some major changes have taken place in the GINA guidelines with regard to the viewpoint and the classification method. Currently, guidelines regarding the management of asthma are based on a historical view of the disease that mostly relates to airflow in the lungs. However, delegates at the summit heard that this takes little account of the inflammatory nature of the disease and whether other parts of the body, besides the lungs, are affected by the same type of inflammation. Also, there is a greater account of the inflammatory nature of the condition and the patient's experience of symptoms, as each patient responds differently to asthma medication. This change in viewpoint was partly based on relevant results of important randomised clinical trials performed during the last five years and they were integrated in the revised GINA guidelines of 2006 (1). Nowadays it is recommended to classify asthma by level of control into Controlled, Partly controlled and Uncontrolled.

The Asthma Insights and Reality in Europe (AIRE) assessed the level of asthma control among Western European asthmatics from the patient's perspective. In this study it was concluded that the patient's perception of asthma control is different from the actual asthma control. Approximately $50 \%$ of the patients in the AIRE study reported severe persistent asthma symptoms, while in the mean time they considered their asthma completely or well under control (2). There seems to be a gap between the reality and the long-term asthma goals as specified by the GINA guidelines. Experts started to realise that through the dissemination of lung function technology, symptoms are transformed from an experience of existential impact into a dip in peak flow, as already reported by Willems (3). In addition, research shows that many patients respond to asthma medications in different ways and, therefore, treatments should be tailored to the individual to maximise the chances of treatment success (4). At first, it was recommended that complex, unstable patients were treated by the physician, and for that 
reason physicians were very reluctant to include these patients in the study. With regard to the new findings in asthma management this implies that the nurse-led telemonitoring programme is probably more beneficial for asthma patients with poor control instead of well-controlled asthmatics.

The cost-effectiveness analysis was performed in an asthma population diagnosed with mild to moderate asthma, based on the GINA guidelines. At the same time the study results showed that the majority of the patients reported to experience very little to no symptoms. Nevertheless the medical consumption was reasonably high. Caution should be taken as the study population represented a relatively small sample. However, in a population with symptoms there is probably more room for improvement and effectiveness. It should be noted that ceiling effects also occurred with regard to the children with chronic conditions as explored in the 'EQ-5D child version study'. This may have contributed to the small effects and may have worsened the discriminant power of the EQ-5D child version.

The programme was developed on its own instead of integrated into the total process of care on a daily basis. Involved were the nurse practitioner, a lung specialist and a paediatrician. The nurse practitioner was available during working hours, and if asthma was experienced as stable, monitor data was transferred once a month. Consequently, the avoidance of asthma attacks can not be the main goal of the nurse. Data were not judged after working hours and the patients were obliged to contact other clinicians during that period. That way the intervention may only partially contribute to the improvement of the total care process, both in terms of health outcomes and costs. The fact is that delivering integrated care has many preconditions. A pitfall is that the focus during the implementation of a new health programme is on isolated interventions, such as medication supply or multidisciplinary cooperation, rather than on the total care process of the patient (3). If integrated care would have been delivered in the total care of our nurse-led telemonitoring programme the cost-effectiveness might improve. Besides patient satisfaction and quality of life, the goal of a programme is to save health care costs and societal costs. Substituting doctors by specialised nurses while using telemonitoring still appears to be a promising method to achieve such savings. In an integrated telemonitoring programme, in which motivated primary and secondary care providers are involved, substitution of care might be easier to obtain and shifting of care might occur less. 


\section{Chapter 7}

As described in a review of Gibson et al. (5), education in asthma-management which involves self-monitoring, regular medical review, and a written action plan appears to improve health outcomes the most. Knowledge about the treatment and the importance of compliance can contribute to the quality of life of the asthma patient. In this study education was minimal. This may have reduced the effectiveness.

\section{Policy recommendations and areas for future research}

In future research it is important that evaluations of new health interventions are not restricted to costs and quality of life. Traditionally, in HTA the costs and effects of a health intervention were the key elements for adequacy. Nevertheless, translating HTA into policy is a highly complex matter. Despite the growth of HTA over the past two decades and the development of different approaches, the direct influence on decision making is still a topic for discussion $(6,7,8,9)$. Van Velden et al. emphasise that though economic evaluations might not be considered the dominant decision criterion for health care decision-making they can offer important evidence on which actions can be based and they provide relevant background information (9). As proposed by Hummel et al., decisions on a new technology should be based on technical, medical, social and economical requirements (10). Although cost-effectiveness analyses are substantial and important in decision making the organisational, ethical and social aspects are elements of HTA should also be regarded. A term to describe this wider form of HTA is Constructive Technology Assessment (CTA). The central telemonitoring intervention in this thesis does not appear to be dominant in terms of cost-effectiveness which is a useful finding. Nevertheless, the process evaluation on the other hand shows that the participants were very satisfied. Outcomes on patient satisfaction and the feasibility of telemedicine programmes deliver cohesive information on the cost-effectiveness of a programme within the target population. The described barriers during the follow-up offer future programme makers useful information. Process evaluation is of high relevance in this procedure, as this enables others to replicate the programme and generate improvements. If the programme was not cost-effective, the process can be adjusted by following the given recommendations. The CTA approach can provide great advantages with regard to health interventions which are in the early adaptor phase. As the number of participants is usually small and the technology use is unstable it is not possible to draw firm conclusions on cost-effectiveness yet (11). Describing the findings on the logistical aspects enhances a more effective implementation. As telemonitoring interventions are recent developments and entail a sociodynamic process, the CTA approach alongside cost-effectiveness evaluations can be of great advantage. 
Furthermore, using modelling techniques based on recent trial data on telemonitoring interventions in asthma, enables the evaluations to be extended beyond what has been observed in a single set of direct observations. Modelling can be applied to decrease the time horizon and reflects what might happen in a different clinical setting or population by using evidence from trials, or systematic reviews (12). In this study the follow-up concerned one year due to practical reasons. Though the intervention was not costeffective after one year, the upward trend in quality of life and the fact that the large intervention costs will possibly decrease in the future, makes modelling an interesting technique for this study setting in the future.

In making decisions among different health care interventions not only the approach of evaluation is determined for the decisions, but also the methods used to evaluate. A recent review confirmed that there is a lack of structured indicators of effectiveness and efficiency in health care models in asthma (13). On one hand the use of process indicators was not uniform and on the other hand there appeared to be no uniform HTA approach. In addition, the methodological quality of cost and cost-effectiveness studies in asthmatics should be improved. For instance, until the societal perspective is applied in economic evaluations, resulting in a cost-utility analysis, it is almost impossible to fully synthesise the evidence of telemonitoring interventions in asthma. The need for uniform guidelines in general was already stated by Gold et al. in 1996 (14). They formulated general recommendations with the purpose to improve the quality and comparability of cost-effectiveness analyses. The OMERACT Economics Working Group already proposed a reference case for economic evaluations in rheumatoid arthritis (15). Gabriel et al. emphasised that standardisation of methods for economic evaluations are essential for defining methodological research agendas (15). Consensus on specific recommendations enhances the conduct and dissemination of methodological research in economic analyses in the rheumatic diseases. The results of this thesis show that such consensus is of high relevance in asthma research. Uniform guidelines for economic evaluations of asthmatics are needed to compare the cost-effectiveness evaluations of telemonitoring interventions.

In this study the programme did not appeare to be cost saving, and especially among children, of limited cost-effectiveness. The decrease of monitor costs would result in a far more cost-effective intervention. Given the fast developments, this decrease in price is expected to take place in the future. As asthma prevalence is increasing and developments in ICT will continuously provide new home monitoring techniques it is 


\section{Chapter 7}

important that these new programmes are evaluated and that the results will be published, whether they are positive or negative. Our advice for these future telemonitoring programmes would be to include education as a main component, involve all the related care providers, and to supply the programme on a continuous base.

Some patients encounter daily telemonitoring as a burden, for instance patients who find the programme too confronting, as they hardly experience symptoms. In this study it was already noticeable that peak flow monitoring twice daily was on average the limit. In a nurse-led monitoring programme lung function data are not only monitored by the patient, but also by a nurse practitioner. One of the advantages of home peak flow monitoring is that patients who do not perceive the severity of their asthma may be alarmed by the objective test results in the monitor or by the nurse. If patients experience hardly any symptoms and objective measurements proof the same, this goal is overreached. Daily telemonitoring is still a promising intervention for more complex patient groups. New results emphasise that the economic and social costs of asthma are mainly caused by poorly controlled conditions and therefore improved management of the respiratory condition would substantially reduce associated costs. Furthermore, nurse practitioners seem to be highly accepted as main care providers in the telemonitoring programme, as of the participants, $84 \%$ did not feel "less safe at all" contacting the nurse instead of the doctor. 


\section{References}

1. GINA Report, Global Strategy for Asthma and Management and Prevention: Revised 2006.

2. Rabe KF, Vermeire PA, Soriano JB, Maier WC. Clinical management of asthma in 1999: The asthma insights and reality in Europe (AIRE) study. Eur Respir J. 2000; 16:802-807.

3. Willems D. Tools of care - Explorations into the semiotics of medical technology, Thesis, 1995, 108.

4. Knobil K, Yancey S, Kral K, Rickard K. Salmeterol multi-center asthma research trial (SMART): results from an interim analysis. Chest. 2003; 124:335S.

5. Gibson PG, Powell H, Coughlan J, Wilson AJ, Abramson M, Haywood P, Bauman A, Hensley MJ, Walters EH. Self-management education and regular practitioner review for adults with asthma (Cochrane Review). Cochrane Database Syst Rev. 2003, Issue 1:CD001117.

6. Oliver A, Mossialos E, Robinson R. Health technology assessment and its influence on health-care priority setting. J Techn Assess Health Care. 2004; 20:1-10.

7. Lilford RJ, Braunholtz D.For debate: The statistical basis of policy: a paradigm shift is overdue. BMJ 1996; 313:603-607.

8. Sheingold $\mathrm{SH}$. Can bayesian methods make data and analyses more relevant to decision makers? A prospective from Medicare. J Techn Assess Health Care. 2001; 17(1):114-122.

9. Van Velden ME, SeverensJL, Novak A. Economic evaluations of healthcare programmes and decision making. Pharmacoeconomics. 2005; 23(11):1075-1082.

10. Hummel MJM, van Rossum W, Verkerke GJ, Rakhorst G. Assessing medical technologies in development: A new paradigm of medical technology assessment. J Techn Assess Health Care. 2000; 16(4):1214-1219.

11. Drummond MF, Jefferson TO. Guidelines for authors and peer reviewers of economic submissions to the BMJ. BMJ. 1996; 313:275-283.

12. Douma KFL, Karsenberg K, Hummel MJM, Bueno-de-Mesquita JM, van Harten WH. Methodology of constructive technology assessment in health care. Int J Techn Assess Health Care. 2007; 23:162-168.

13. Steuten L, Lemmens K, Vrijhoef B. Health technology assessment of asthma disease management programs. Allergy Clin Immunol. 2007; 7:242-248.

14. Gold M, Siegel JE, Rusell LB, Weinstein MC. Cost-effectiveness in health and medicine. New York: Oxford University Press; 1996.

15. Gabriel S, Drummond M, Maetzel A, Boers M, Coyle D, Welch V, Tugwell P. OMERACT 6 Economics Working Group report: a proposal for a reference case for economic evaluation in rheumatoid arthritis. J Rheumatol. 2003; 30(4):886-890. 
Summary 


\section{Summary}

Asthma is a chronic condition which cannot be cured, but can be controlled. Asthmatics should be able to live an active life with the appropriate care. Since the disease is chronic, asthma management is continuously aimed at reducing symptoms to a minimum. Pharmacological management is important in the treatment of asthma. However, asthma care should also entail interventions, training, and skills by which patients can effectively take care of themselves. For that reason self-monitoring interventions are an established aspect of asthma management in both children and adults. With regard to care improvement, it is important that these asthma programmes are properly evaluated and that the results are published.

In this thesis the effectiveness and cost-effectiveness of a nurse-led telemonitoring programme based on peak flow measurements are compared to regular outpatient care. Besides calculating the effectiveness and cost-effectiveness of a new programme, there are other important aspects which should be explored to determine if a programme is worth implementing. Important outcomes for success are also the patient's satisfaction and the feasibility of the programme. These aspects were explored in a process evaluation. In addition, a multi-attribute utility instrument to measure health-related quality of life in children was investigated.

Chapter 2 systematically reviews available literature on the costs and the costeffectiveness of self-management interventions based on peak flow monitoring interventions in asthma. The review includes 21 economic evaluations. A detailed summary was given of the applied interventions, health outcomes and direct and indirect costs. The methodological and economic quality of the studies was assessed by the use of a checklist. In 13 of the 17 economic evaluations that analysed health outcomes, at least one of the reported health outcomes improved statistically significantly after the intervention. In only 2 studies the total costs were higher in the intervention group. Given the results of the studies the use of self-management programmes based on peak flow monitoring in asthmatics seems to be cost effective or even cost saving. However, this review should be interpreted with caution, because of the relatively poor methodological quality of the studies and the difference in methods. To draw valid conclusions in cost and cost-effectiveness studies of self-management programmes based on peak flow monitoring in asthmatics, the methodological quality of these evaluations should be improved and uniform guidelines are needed. 
In chapter 3 the results of the process evaluation of the nurse-led telemonitoring programme are described. The process indicators include feasibility, consistency of PEF tests, compliance with the intervention and study protocols, and patient satisfaction.

Less then half of the asthmatic outpatients were willing to participate. Although technical and logistical problems did occur, the dropout rate was low. At least $75 \%$ of the PEF manoeuvres were valid for two third of 20 patients and the compliance with the study protocol was high. Two PEF tests were required per day, but the actual number of recorded PEF tests averaged less. Consequently, this implies that the maximum level of burden with regard to PEF tests a day was reached. Nevertheless, the patient satisfaction was high and, after one year, most of the participants chose to continue their participation. This finding is in harmony with the answer of most patients that they felt safer while using the monitor. The chapter shows that the programme is feasible, reliable, and satisfying in a motivated group of mild to moderate asthmatics.

Chapter 4 presents the effectiveness results of the randomised controlled trial comparing the nurse-led telemonitoring programme to regular care. The effects on asthma symptoms, asthma-specific quality of life, and medical consumption are described. Also, the relationships between these effects are evaluated, as they may facilitate the interpretation of research findings. The study population represented mild to moderate asthmatics. The asthma symptoms, asthma-specific quality of life, and medical consumption showed improvement in follow-up, but no statistically significant differences between the groups were observed. Moderate to high correlations were found between the PEF and the FEV1, between the symptom scores, between corticosteroids and beta2 agonists, and between the domains of asthma-specific quality of life.

The most remarkable was the low, but statistically significant, correlation between asthma-specific quality of life (daily functioning) and the self-reported beta-2 agonists (medicines used to relieve an asthma attack). This relationship indicates that patients' perception of asthma-specific quality of life should be a key element in asthma telemonitoring programmes.

In chapter 5 the usefulness of the EQ-5D for assessing quality of life in economic evaluations among children with asthma, diabetes, a rheumatic disorder, or a speech/language/hearing disorder was examined. Up to now the validity of the EQ-5D child version has not been investigated in children with chronic conditions. To explore the validity and reliability of this multi-attribute utility instrument the feasibility, construct validity, discriminant power, and test-retest reliability were examined. 


\section{Summary}

The feasibility was on average good and seemed the lowest among children with a speech/language/hearing disorder. Correlations between the EQ-5D child version and another generic quality of life instrument, the TACQOL, were low to moderate which suggests that the instruments measure different concepts. The on average very high quality of life in the study populations lead to more ceiling effects in the EQ-5D than in the TACQOL. The discriminant power was the highest between children with a rheumatic disorder and the other conditions. The 2-week test-retest reliability of the EQ-5D was considerable lower in children in which the course of the condition was more variable. In conclusion, more research is needed to investigate multi-attribute instruments in children, as there is a growing demand for these instruments, and it appears that the EQ$5 \mathrm{D}$ is not directly suitable for children.

In chapter 6 the results of the trial-based cost-effectiveness analysis of the nurse-led telemonitoring programme are presented from a societal and a health care perspective. No statistically significant differences were found between the groups with regard to generic quality of life. Overall, the mean health care costs per patient were higher in the intervention group than in the control group. The costs due to loss of productivity at paid labour were on average five times higher among the patients in the intervention group, than among the patients in the control group. The intervention costs mainly caused the cost difference between the groups. The mean incremental cost-effectiveness ratio among adults was $€ 15,366 /$ QALY gained from the health care perspective and $€$ 31,035 /QALY gained from the societal perspective. Among children, from the health care perspective the mean ICER was $€ 58,726$ /QALY gained and from the societal perspective $€ 59,071$ /QALY gained. If the monitor costs were excluded the incremental costs decreased strongly among both the adults and the children. Considering the results of this study the intervention is certainly not cost saving, and especially among children, of limited cost-effectiveness.

In the end, chapter 7 provides a general discussion concerning the results of the study. The main findings and methodological findings are summarised. Furthermore, the generalisation of the study results and the limitations of the study are discussed. In addition, policy recommendations for future research are made. 
Samenvatting 


\section{Samenvatting}

Astma is een chronische aandoening, die niet te genezen is. Met de passende zorg kunnen symptomen tot een minimum worden gereduceerd en zou een astma patiënt een actief leven moeten kunnen leiden. Astma management is hierbij van groot belang en dient doeltreffend en efficiënt te zijn. Enerzijds vormt een medicamenteuze behandeling een essentieel onderdeel van astma management. Anderzijds vormen interventies, training en het aanleren van technieken waardoor de patiënt effectief voor zichzelf kan zorgen belangrijke onderdelen. Zelfmonitoring programma's zijn daarom een onmiskenbaar aspect van astma management voor zowel kinderen als volwassenen. Om zorg verbetering te bewerkstelligen is het belangrijk dat astma programma's zorgvuldig worden geëvalueerd en de resultaten gepubliceerd.

In dit proefschrift worden de effectiviteit en kosteneffectiviteit van een telemonitoring programma gebaseerd op peak flow metingen met een verpleegkundige als centrale zorgverlener vergeleken met de gebruikelijke poliklinische zorg. Behalve het berekenen van de effectiviteit en de kosteneffectiviteit van een nieuw programma zijn er ook andere belangrijke aspecten die onderzocht dienen te worden om te bepalen of een programma met succes geïmplementeerd kan worden. Andere belangrijke factoren voor succes zijn de tevredenheid van de patiënt en de bruikbaarheid van het programma. Deze factoren zijn onderzocht in een proces evaluatie. Aanvullend is een multidimensioneel meetinstrument voor de kwaliteit van leven bij kinderen onderzocht.

Hoofdstuk 2 betreft een review. Hierin worden de kosten en de kosteneffectiviteit van zelfmanagement gebaseerd op peak flow monitoring interventies in astma geanalyseerd. Dit vond plaats door middel van een systematische review van 21 economische evaluaties. Een gedetailleerde samenvatting werd gegeven van de gehanteerde interventies, de gezondheidsuitkomsten en de directe en indirecte kosten. De economische en methodologische kwaliteit van de studies werden bepaald door het gebruik van een checklist. $\mathrm{Bij} 13$ van de 17 economische evaluaties die gezondheidsuitkomsten analyseerden was voor minimaal één van de gerapporteerde gezondheidsuitkomsten na de interventie een statistisch significante verbetering zichtbaar. In slechts twee studies waren de totale kosten hoger in de interventie groep. Deze resultaten lijken aan te duiden dat zelfmanagement programma's gebaseerd op peak flow monitoring bij astma patiënten kosteneffectief of zelfs kostenbesparend zijn. Niettemin moet men de resultaten van de review voorzichtig interpreteren vanwege de relatief slechte methodologische kwaliteit van de studies en het verschil in de 
gehanteerde methoden. Om betrouwbare conclusies te kunnen trekken uit kosten en kosteneffectiviteit studies over astma zelfmanagement programma's gebaseerd op peak flow monitoring interventies, zal de methodologische kwaliteit van deze evaluaties moeten verbeteren en zijn er uniforme richtlijnen nodig.

In hoofdstuk 3 worden de resultaten van het proces van het telemonitoring programma met een verpleegkundige als begeleider geëvalueerd. De proces indicatoren betreffen de uitvoerbaarheid, de stabiliteit van de peak flow metingen, de compliance met het interventie protocol en het studie protocol en de patiënten tevredenheid. Minder dan de helft van de poliklinische astma patiënten was bereid mee te werken. Ondanks dat technische en logistische problemen plaats vonden was het uitval percentage laag. Minstens $75 \%$ peak flow manoeuvres waren valide bij tweederde van de 20 patiënten en de compliance met het studie protocol was hoog. Gevraagd werd naar twee peak flow testen per dag, maar het daadwerkelijk uitgevoerde peak flow testen lag gemiddeld lager. Dit impliceert dat de maximale belasting met betrekking tot het uitvoeren van peak flow testen was behaald. Desalniettemin was de patiënten tevredenheid hoog en na één jaar wilden de meeste deelnemers doorgaan met hun deelname. Deze bevinding komt overeen met het antwoord van de meeste patiënten dat ze zich veiliger voelden tijdens het gebruik van de monitor. In het hoofdstuk wordt aangetoond dat het programma uitvoerbaar, betrouwbaar en bevredigend is in een gemotiveerde groep met patiënten met milde tot matige astma.

In hoofdstuk 4 worden de effecten van het telemonitoring programma geleid door een verpleegkundige vergeleken met de gebruikelijke poliklinische zorg. Hierbij worden de effecten op astma symptomen, astma specifieke kwaliteit van leven en medische consumptie beschreven. De relaties tussen de effecten werden tevens geëvalueerd, omdat deze de interpretatie van de onderzoeksbevindingen kunnen vergemakkelijken. De studiepopulatie representeerde milde tot matig astma patiënten. Wat betreft de astma symptomen, astma specifieke kwaliteit van leven en de medische consumptie was in beide groepen een verbetering in de tijd zien. Aan de andere kant werd er geen statistisch significant verschil tussen de groepen waargenomen. Matige tot hoge correlaties werden gevonden tussen de PEF en de FEV1, tussen de astma symptomen, tussen de corticosteroïden en de beta-2 agonisten en tussen de domeinen van de astma specifieke kwaliteit van leven. Het meest opvallende was de lage statistisch significante 


\section{Samenvatting}

correlatie tussen astma specifieke kwaliteit van leven (dagelijks functioneren) en het gerapporteerde gebruik van beta-2 agonisten (luchtverwijdend medicijn voor tijdens een astma aanval). Deze relatie duidt aan dat de perceptie van de patiënt over astma specifieke kwaliteit van leven het hoofdelement zou moeten zijn in telemonitoring programma's in astma.

Hoofdstuk 5 gaat verder in op de bruikbaarheid van de EQ-5D kind versie in economische evaluaties bij het bepalen van de kwaliteit van leven in van kinderen met astma, een reumatische aandoening of spraak-taal-gehoor moeilijkheden. Tot nu toe is de validiteit van de EQ-5D kind versie nog niet onderzocht bij kinderen met een chronische aandoening. Om de validiteit en betrouwbaarheid van dit multidimensionele meetinstrument te bepalen werden de bruikbaarheid, de kruis validiteit, het discriminerend vermogen en de test-hertest betrouwbaarheid onderzocht.

De bruikbaarheid was gemiddeld goed en leek het laagste bij kinderen met spraak-taalgehoor moeilijkheden. De correlaties van de EQ-5D kind versie met een ander generieke kwaliteit van leven meetinstrument, TACQOL, waren laag tot gemiddeld. Dit suggereert dat de instrumenten een ander concept meten. De gemiddeld zeer hoge kwaliteit van leven in de studie populaties leidde in de EQ-5D tot meer plafond effecten dan in de TACQOL. Het discriminerende vermogen was het hoogste tussen de kinderen met een reumatische aandoening en de andere aandoeningen. De test-hertest betrouwbaarheid van de EQ-5D was aanzienlijk lager bij kinderen waar het ziekteverloop meer veranderlijk was. De conclusie is dat er meer onderzoek nodig is naar multidimensionele meetinstrumenten voor kinderen, aangezien hier een groeiende vraag naar is en blijkt dat de EQ-5D niet meteen toepasbaar is bij kinderen.

Hoofdstuk 6 geeft een overzicht van de resultaten van de kosteneffectiviteitanalyse van het telemonitoring programma geleid door een verpleegkundige. Dit heeft plaats gevonden vanuit het maatschappelijke en het gezondheidszorg perspectief. Geen statistisch significante verschillen werden gevonden tussen de groepen met betrekking tot de kwaliteit van leven. Over het algemeen waren de gemiddelde gezondheidszorg kosten per patiënt hoger in de interventie groep dan in de controle groep. De kosten van arbeid gerelateerde productiviteitsverliezen lagen gemiddeld vijf keer hoger bij de patiënten in de interventiegroep in vergelijking met de controle groep. Dit kostenverschil tussen de groepen werd voornamelijk veroorzaakt door de kosten van de interventie. De 
gemiddelde incrementele kosteneffectiviteitratio was $€ 15,366$ per QALY bij de volwassenen vanuit het gezondheidszorg perspectief en $€ 31,035$ per QALY vanuit het maatschappelijke perspectief. Bij de kinderen was de gemiddelde incrementele kosteneffectiviteitratio $€ 58,726$ per QALY vanuit het gezondheidszorg perspectief en vanuit het maatschappelijke perspectief $€ 59,071$ per QALY. Als de monitor kosten werden geëxcludeerd verlaagden de incrementele kosten aanzienlijk, bij zowel de volwassenen als de kinderen. De studie resultaten tonen aan dat de interventie zeker niet kostenbesparend is en vooral bij kinderen beperkt kosteneffectief is.

Tot slot geeft hoofdstuk 7 een overzicht van de studie resultaten door middel van een algemene discussie. Hierin worden de belangrijkste bevindingen samengevat en de methodologische beperkingen besproken. Vervolgens worden de studie resultaten gegeneraliseerd en de studie beperkingen aangehaald. Aanvullend worden politieke aanbevelingen gegeven voor toekomstig onderzoek. 


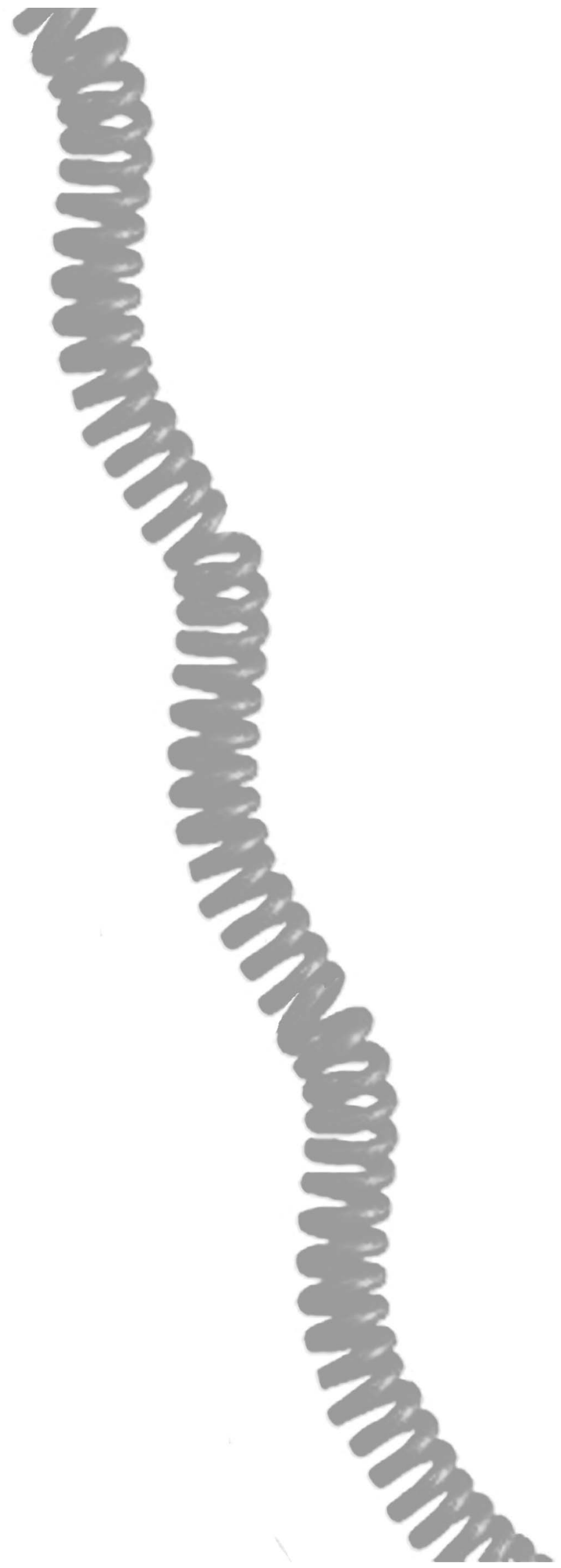

Dankwoord 
De volgende personen hebben allemaal op hun eigen manier bijgedragen aan dit proefschrift en daarom wil ik ze hiervoor bedanken.

Allereerst wil ik mijn promotoren Hans Severens en Miel Wouters en mijn co-promoteren Manuela Joore en Han Hendriks bedanken. Zonder jullie inzet was dit proefschrift uiteraard nooit tot stand gekomen.

Manuela, ik heb onze intensieve samenwerking en je begeleiding erg gewaardeerd. Je kon me enorm geruststellen en motiveren. Ik vind het fijn dat jij mijn co-promotor bent geweest.

Hans, hartelijk bedankt voor het delen van je kennis over MTA en alle ondersteuning die je mij hebt gegeven als promotor. Miel Wouters wil ik bedanken voor zijn waardevolle bijdrage als promotor en bij het tot stand komen en het voltooien van het onderzoek en dit proefschrift. Han, co-promotor, bedankt voor je inhoudelijke bijdrage, en speciaal voor je inzet bij het vinden van ouders en kinderen die wilden deel nemen aan het onderzoek. Jullie adviezen en inzichten hebben veel bijgedragen aan dit proefschrift. Bedankt voor de prettige samenwerking.

Dr GJ Wesseling, Dr Q Jöbsis, Dr E Dompeling, Dr L Anteunis, Dr A van Henten, wil ik bedanken voor hun bijdrage, in het bijzonder bij het opstarten van het onderzoek en bij de patiënten rekrutering in het academisch ziekenhuis Maastricht.

Daarnaast wil ik de medewerkers van de Mgr Hanssenschool en het Diabetes Centrum te Blaricum bedanken voor hun bijdrage bij de rekrutering van ouders en kinderen. Dit geldt tevens voor $\mathrm{Dr} J$ Heijnens en de nurse practitioner $\mathrm{T}$ Dauven van het Maaslandziekenhuis te Sittard.

Fred Nieman, bedankt dat je bereid was mij te begeleiden bij de statistische analyses. Ik heb veel geleerd van jouw hulp bij de interpretatie van mijn resultaten.

De leden van de beoordelingscommissie, Prof $\mathrm{Dr} L$ Zimmermann (voorzitter), Prof $\mathrm{Dr} J$ de Jongste, Prof Dr J Knottnerus, Dr T Schermer en Prof Dr R de Wit wil ik bedanken voor de kritische beoordeling van mijn proefschrift.

Het bijwonen en participeren in de bijeenkomsten van de MTA-werkgroep hebben geleid tot bruikbare ideëen voor mijn eigen onderzoek en een bredere kijk op onderzoek in het algemeen. Bedankt hiervoor. 
Raymond van Duurling, gespecialiseerd verpleegkundige, door met jou 'mee te lopen' heb ik het programma niet alleen vanachter mijn bureau, maar ook in uitvoering gezien. Jou bezig zien, bevestigt maar weer eens dat verpleegkunde een mooi beroep is.

Janneke en Claire, jullie als paranimfen was een makkelijke keus. Janneke, jij zorgde voor een gezellige werksfeer op onze kamer. Rustgevend en behulpzaam, werkt goed bij iemand die soms een beetje geremd moet worden in haar vrolijkheid.

Claire, aangezien we op werkgebied in het zelfde schuitje zaten heb ik veel steun aan je gehad. Ik moet zeggen dat ik de grappigste momenten toch aan jou relateer. Wedden dat we als we 'uitgeparanimft' zijn bij elkaar, nog heel veel lol zullen hebben.

Martin Prins, hoofd afdeling KEMTA en voorzitter capaciteitsgroep Epidemiologie, bedankt voor je vertrouwen in mij. Vroegere collega's van de afdeling KEMTA bedankt voor de gezelligheid en jullie betrokkenheid. Zoals jullie nu al weten zal ik nog geregeld langs komen om even gezellig bij te kletsen.

De astma patiënten en hun ouders wil ik bedanken voor hun medewerking aan het onderzoek en dan met name voor het invullen van de vragenlijsten en hun vriendelijke reacties.

Lieve pap en mam, familie en vrienden. Bedankt dat jullie er altijd voor mij zijn en het leven zo gezellig maken. Zonder jullie zou deze dag niet zo speciaal zijn. Jos, de allerlaatste zin is voor jou. 


\section{Curriculum vitae}

Daniëlle Willems werd geboren op 3 juni te Melick-Herkenbosch. Zij behaalde het Gymnasium diploma in 1993 aan de Stedelijk Scholengemeenschap te Maastricht. $\mathrm{Na}$ eerst twee jaar Rechten te hebben gestudeerd aan de Universiteit van Amsterdam, begon zij in 1995 aan de opleiding tot Verpleegkunde (HBO-V) aan de Hogeschool Zuyd. In 1999 voltooide zij deze opleiding en vervolgens volgde zij de verkorte opleiding Gezondheidswetenschappen aan de Universiteit Maastricht met als afstudeerrichting Zorgwetenschappen. In 2002 behaalde zij haar diploma en hierna was ze werkzaam bij de afdeling Klinische Epidemiologie en Medical Technology Assessment (KEMTA) in het academisch ziekenhuis Maastricht. Tot 2005 was zij zowel onderzoeksassistent op een project van de vakgroep Psychiatrie en Neuropsychologie als wetenschappelijk onderzoeker voor het onderwerp dat ten grondslag ligt aan dit proefschrift. Hierna vervolgde zij fulltime haar promotieonderzoek in samenwerking met de afdelingen KEMTA, Longziekten en Kindergeneeskunde van het academisch ziekenhuis Maastricht. Sinds 2007 is zij werkzaam als onderzoeker binnen de capaciteitsgroep Epidemiologie van de Universiteit Maastricht. 
De liefde die laat komt, brengt vaak groot geluk. 
